SRNL-STI-2012-00309

Revision 0

Keywords: Salt, Oxalic

Acid, leaching test

Retention: Permanent

\title{
Characterization of Tank 16H Annulus Samples Part II: Leaching Results
}

M. S. Hay

S. H. Reboul

June 2012

Savannah River National Laboratory

Savannah River Nuclear Solutions, LLC Aiken, SC 29808

Prepared for the U.S. Department of Energy under contract number DE-AC09-08SR22470.

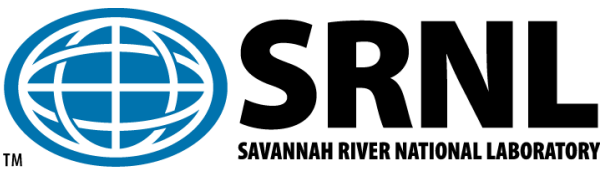


SRNL-STI-2012-00309

Revision 0

\section{DISCLAIMER}

This work was prepared under an agreement with and funded by the U.S. Government. Neither the U.S. Government or its employees, nor any of its contractors, subcontractors or their employees, makes any express or implied:

1. warranty or assumes any legal liability for the accuracy, completeness, or for the use or results of such use of any information, product, or process disclosed; or

2. representation that such use or results of such use would not infringe privately owned rights; or

3. endorsement or recommendation of any specifically identified commercial product, process, or service.

Any views and opinions of authors expressed in this work do not necessarily state or reflect those of the United States Government, or its contractors, or subcontractors.

\section{Printed in the United States of America}

\section{Prepared for}

U.S. Department of Energy 
SRNL-STI-2012-00309

Revision 0

\section{REVIEWS AND APPROVALS}

\section{AUTHORS:}

M. S. Hay, SRNL/ACP

S. H. Reboul, SRNL/PTP

TECHNICAL REVIEW:

K. Adu-Wusu, SRNL/ACP

APPROVAL:

F. M. Pennebaker, SRNL/ACP

S. L. Marra, SRNL/E\&CPT Research Programs

R. H. Spires, Waste Removal \& Tank Closure Projects

C. L. Atseff, Tank Farm \& Closure Engineering 
SRNL-STI-2012-00309

Revision 0

\section{EXECUTIVE SUMMARY}

The closure of Tank $16 \mathrm{H}$ will require removal of material from the annulus of the tank. Samples from Tank $16 \mathrm{H}$ annulus were characterized and tested to provide information to evaluate various alternatives for removing the annulus waste.

The analysis found all four annulus samples to be composed mainly of $\mathrm{Si}, \mathrm{Na}$, and $\mathrm{Al}$ and lesser amounts of other elements. The XRD data indicate quartz $\left(\mathrm{SiO}_{2}\right)$ and sodium aluminum nitrate silicate hydrate $\left(\mathrm{Na}_{8}\left(\mathrm{Al}_{6} \mathrm{Si}_{6} \mathrm{O}_{24}\right)\left(\mathrm{NO}_{3}\right)_{2} \cdot 4 \mathrm{H}_{2} \mathrm{O}\right)$ as the predominant crystalline mineral phases in the samples. The XRD data also indicate the presence of crystalline sodium nitrate $\left(\mathrm{NaNO}_{3}\right)$, sodium nitrite $\left(\mathrm{NaNO}_{2}\right)$, gibbsite $\left(\mathrm{Al}(\mathrm{OH})_{3}\right)$, hydrated sodium bicarbonate $\left(\mathrm{Na}_{3} \mathrm{H}\left(\mathrm{CO}_{3}\right)_{2} \cdot 2 \mathrm{H}_{2} \mathrm{O}\right)$, and muscovite $\left(\mathrm{KAl}_{2}\left(\mathrm{AlSi}_{3} \mathrm{O}_{10}\right)(\mathrm{OH})_{2}\right)$.

Based on the weight of solids remaining at the end of the test, the water leaching test results indicate $20-35 \%$ of the solids dissolved after three contacts with an approximately 3:1 volume of water at $45^{\circ} \mathrm{C}$. The chemical analysis of the leachates and the XRD results of the remaining solids indicate sodium salts of nitrate, nitrite, sulfate, and possibly carbonate/bicarbonate make up the majority of the dissolved material. The majority of these salts were dissolved in the first water contact and simply diluted with each subsequent water contact. The water leaching removed large amounts of the uranium in two of the samples and approximately $1 / 3$ of the ${ }^{99}$ Tc from all four samples. Most of the other radionuclides analyzed showed low solubility in the water leaching test.

The oxalic acid leaching test result indicate approximately $34-47 \%$ of the solids in the four annulus samples will dissolve after three contacts with an approximately 3:1 volume of acid to solids at $45^{\circ} \mathrm{C}$. The same sodium salts found in the water leaching test comprise the majority of dissolved material in the oxalic acid leaching test. However, the oxalic acid was somewhat more effective in dissolving radionuclides than the water leach. In contrast to the water leaching results, most constituents continued to dissolve during subsequent cycles of oxalic acid leaching.

The somewhat higher dissolution found in the oxalic acid leaching test versus the water leaching test might be offset by the tendency of the oxalic acid solutions to take on a gellike consistency. The filtered solids left behind after three oxalic acid contacts were sticky and formed large clumps after drying. These two observations could indicate potential processing difficulties with solutions and solids from oxalic acid leaching. The gel formation might be avoided by using larger volumes of the acid. Further testing would be recommended before using oxalic acid to dissolve the Tank $16 \mathrm{H}$ annulus waste to ensure no processing difficulties are encountered in the full scale process. 


\section{TABLE OF CONTENTS}

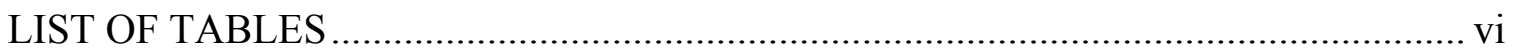

LIST OF FIGURES …………………............................................................ vii

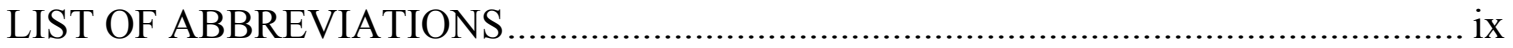

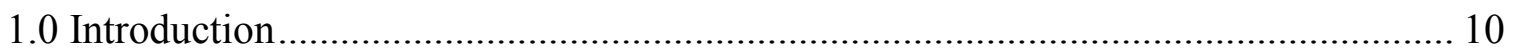

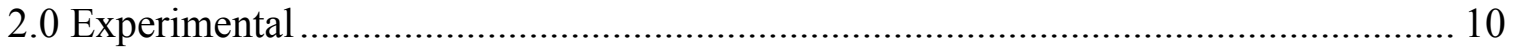

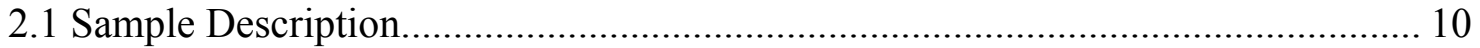

2.2 Sample Preparation and Analysis ..................................................................... 10

2.3 Water and Oxalic Acid Leaching Tests ................................................................. 11

3.0 Analytical Results and Discussion........................................................................ 13

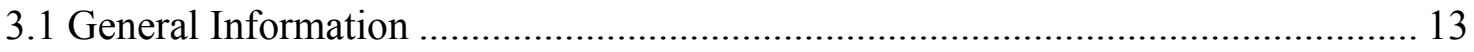

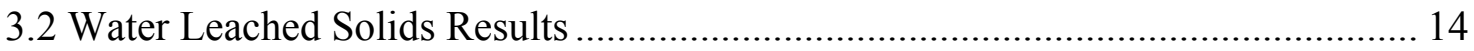

3.3 Oxalic Acid Leachate Results........................................................................ 14

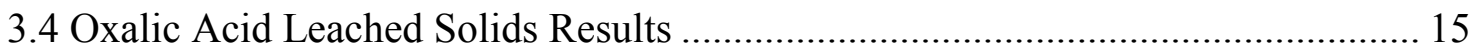

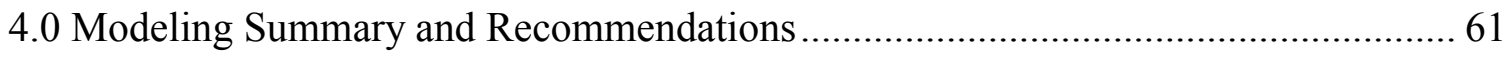

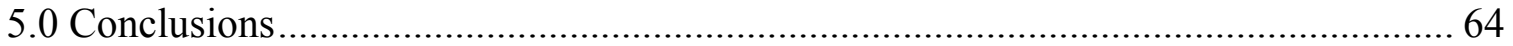

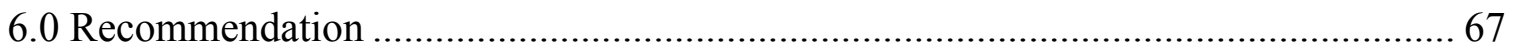

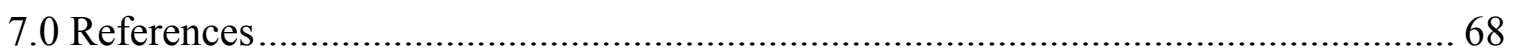

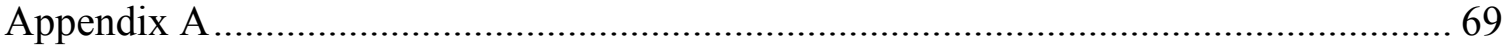


SRNL-STI-2012-00309

Revision 0

\section{LIST OF TABLES}

Table 2-1. Sampling Locations of Tank 16H Annulus Samples ................................. 12

Table 2-2. Composition of the Analytical Reference Glass-1 Standard. ...................... 12

Table 3-1. Abbreviations for Analytical Methods used in Data Tables ....................... 14

Table 3-2. Composition of the Water Leached Solids from Tank 16H Annulus Sample

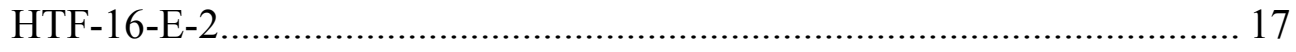

Table 3-3. Composition of the Water Leached Solids from Tank 16H Annulus Sample HTF-16-N-1 ............................................................................... 20

Table 3-4. Composition of the Water Leached Solids from Tank 16H Annulus Sample HTF-16-S-2 ............................................................................ 23

Table 3-5. Composition of the Water Leached Solids from Tank 16H Annulus Sample HTF-16-W-1 .............................................................................. 26

Table 3-6. Composition of the Oxalic Acid Leachates from Tank 16H Annulus Sample HTF-16-E-2 from Three Leaching Cycles................................................ 29

Table 3-7. Composition of the Oxalic Acid Leachates from Tank 16H Annulus Sample HTF-16-N-1 from Three Leaching Cycles .............................................. 32

Table 3-8. Composition of the Oxalic Acid Leachates from Tank 16H Annulus Sample HTF-16-S-2 from Three Leaching Cycles ................................................ 35

Table 3-9. Composition of the Oxalic Acid Leachates from Tank 16H Annulus Sample HTF-16-W-1 from Three Leaching Cycles ........................................... 38

Table 3-10. Composition of the Oxalic Acid Leached Solids from Tank 16H Annulus Sample HTF-16-E-2

Table 3-11. Composition of the Oxalic Acid Leached Solids from Tank 16H Annulus Sample HTF-16-N-1

Table 3-12. Composition of the Oxalic Acid Leached Solids from Tank 16H Annulus Sample HTF-16-S-2

Table 3-13. Composition of the Oxalic Acid Leached Solids from Tank 16H Annulus Sample HTF-16-W-1

Table 3-14. XRD Result for the Oxalic Acid Leached Solids from the Tank 16H Annulus Samples-Estimated Mineral Phase Composition

Table 4-1. Species Chosen for the ESP Chemistry Model of Tank 16 Annulus Solids 61 
Table 5-1. Summary of the Composition of the As-Received, Water Leached, and Oxalic Acid Leached Solids from Tank 16H Annulus Samples (Wt\% Total Dried Solids)....

Table 5-2. Estimated Percent of Solids Dissolved in the Water Leaching and Oxalic Acid Leaching Tests on Tank 16H Annulus Samples ......

Table 5-3. Summary of the Estimated Percentage Dissolved for each Radionuclide from the Water Leaching Test on Tank 16H Annulus Samples .................. 66

Table 5-4. Summary of the Estimated Percentage Dissolved for each Radionuclide from the Oxalic Acid Leaching Test on Tank 16H Annulus Samples ........ 66

Table A-1. Composition of Blanks and Standards for the Water Leached Solids from Tank 16H Annulus Sample.

Table A-2. Composition of Blanks and Standards for the Oxalic Acid Leached Solids from Tank 16H Annulus Sample 
SRNL-STI-2012-00309

Revision 0

\section{LIST OF FIGURES}

Figure 3-1 Oxalic Acid Leached Solids from the Tank 16H Annulus Samples............ 53

Figure 3-2 Oxalic Acid Leached Solids from Tank 16H Annulus Sample HTF 16-E-2

Figure 3-3 Oxalic Acid Leached Solids from Tank 16H Annulus Sample HTF 16-N-1

Figure 3-4 Oxalic Acid Leached Solids from Tank 16H Annulus Sample HTF 16-S-255

Figure 3-5 Oxalic Acid Leached Solids from Tank 16H Annulus Sample HTF 16-W-1

Figure 3-6 XRD Results of the Oxalic Acid Leached Solids for HTF-16E2-1 ............ 57

Figure 3-7 XRD Results of the Oxalic Acid Leached Solids for HTF-16E2-2 ............ 57

Figure 3-8 XRD Results of the Oxalic Acid Leached Solids for HTF-16N1-1 „.......... 58

Figure 3-9 XRD Results of the Oxalic Acid Leached Solids for HTF-16N1-2 _.......... 58

Figure 3-10 XRD Results of the Oxalic Acid Leached Solids for HTF-16S2-1 ........... 59

Figure 3-11 XRD Results of the Oxalic Acid Leached Solids for HTF-16S2-2 ........... 59

Figure 3-12 XRD Results of the Oxalic Acid Leached Solids for HTF-16W1-1 ......... 60

Figure 3-13 XRD Results of the Oxalic Acid Leached Solids for HTF-16W1-2 .......... 60 
SRNL-STI-2012-00309

Revision 0

\section{LIST OF ABBREVIATIONS}

$\begin{array}{ll}\text { AD } & \text { Analytical Development } \\ \text { BS } & \text { Beta Spectroscopy } \\ \text { ESP } & \text { Environmental Simulation Program } \\ \text { GS } & \text { Gamma Spectroscopy } \\ \text { IC } & \text { Ion Chromatography } \\ \text { IE } & \text { Inductively Coupled Plasma-Emission Spectroscopy } \\ \text { IM } & \text { Inductively Coupled Plasma-Mass Spectrometry } \\ \text { MSE } & \text { Mixed Solvent Electrolyte } \\ \text { MSEPUB } & \text { Mixed Solvent Electrolyte Public Database } \\ \text { SA } & \text { Separation/Alpha Spectroscopy } \\ \text { SRNL } & \text { Savannah River National Laboratory } \\ \text { SRR } & \text { Savannah River Remediation } \\ \text { TH } & \text { Titration for Free Hydroxide } \\ \text { TTR } & \text { Technical Task Request } \\ \text { XRD } & \text { X-Ray Diffraction }\end{array}$


SRNL-STI-2012-00309

Revision 0

\subsection{Introduction}

The closure of Tank $16 \mathrm{H}$ requires removal of material from the annulus of the tank. Savannah River Remediation (SRR) requested that the Savannah River National Laboratory (SRNL) provide information to evaluate various alternatives for removing the annulus waste. Samples from Tank $16 \mathrm{H}$ annulus were obtained and sent to SRNL to determine the chemical and radiochemical composition and to determine the leaching characteristics of the material. The work was conducted as stated in the task plan ${ }^{1}$ developed from the Technical Task Request (TTR). ${ }^{2}$ The results from the analysis of the initial as-received solids, the duct sample ${ }^{137} \mathrm{Cs}$ concentration, results of the water leachate analysis, and annulus cleaning recommendations were previously reported. ${ }^{3}$ This report documents the composition of the oxalic acid leachates, the composition of the solids remaining after water and oxalic acid leaching, and provides recommendations for the chemical species to use for thermodynamic modeling. Several other reports provide additional characterization information on the material in the Tank $16 \mathrm{H}$ annulus. ${ }_{4,6}$

\subsection{Experimental}

\subsection{Sample Description}

A total of four samples were obtained from the Tank $16 \mathrm{H}$ annulus. Table $2-1$ provides the riser sampling location in the annulus and the dates the samples were received at SRNL. The material obtained for each of the four samples appeared to be very dry and powdery and ranged in color from light grey to dark grey. Additional details on the samples can be found in a previous report. . $^{3}$

\subsection{Sample Preparation and Analysis}

For analysis by X-ray Diffraction (XRD), small amounts of the solids were placed in a shielded bottle and sent to Analytical Development (AD). Samples for XRD were analyzed in duplicate. The initial as-received solids were not washed, dried, or ground prior to sub-sampling for XRD analysis. Solids from the water and oxalic acid leaching tests were rinsed on the filter three times with small amounts of water to replace the interstitial liquid and dried in an oven at $110^{\circ} \mathrm{C}$ to constant weight before XRD analysis.

After drying to constant weight in an oven at $110^{\circ} \mathrm{C}$, the solids from the leaching tests were prepared for analysis using two methods. An aqua regia digestion and a standard fusion digestion with sodium peroxide followed by uptake in nitric acid were used to dissolve the dried solids from the samples. The digested solids from each method were diluted to reduce activity and allow removal from the Shielded Cells. All sample preparations were conducted in duplicate. A digestion of a glass standard containing many of the elements found in tank samples was prepared concurrently with the sample digestions. Table 2-2 lists the composition of the Analytical Reference Glass-1 glass 
standard. ${ }^{7}$ A reagent blank was prepared concurrently with the sample preparations consisting of the digestion reagents using the same manipulations and dilutions conducted on the sample.

\subsection{Water and Oxalic Acid Leaching Tests}

Leaching tests were conducted on the Tank $16 \mathrm{H}$ annulus samples to determine the solubility of the sample material in water and $4 \mathrm{wt} \%$ oxalic acid. Approximately 50 grams of the as-received solids from each sample were contacted with $150 \mathrm{~mL}$ of leaching solution for $\sim 24$ hours. The tests were conducted in a shaker oven maintained at $45{ }^{\circ} \mathrm{C}$ throughout the duration of the test. After $\sim 24$ hours with moderate agitation, the shaker was switched off and the solids allowed to settle. The liquid was decanted from the bottle as close to the settled solids interface as possible without disturbing the solids. Another $150 \mathrm{~mL}$ of leaching solution was then added to the solids and the mixtures agitated at $45^{\circ} \mathrm{C}$ for another 24 hours. A third contact was conducted in the same manner.

All of the decanted solutions were filtered through a $0.45 \mu$ m nylon filter to remove fines carried over during the decanting. Single aliquots of each decanted liquid from the leaching tests were removed without dilution and sent to $\mathrm{AD}$ for analysis.

The solids remaining from the leaching tests were filtered through a $0.45 \mu \mathrm{m}$ nylon filter and rinsed on the filter with three $30 \mathrm{~mL}$ portions of de-ionized water to remove the interstitial liquid. The solids were dried in a $110^{\circ} \mathrm{C}$ oven overnight and prepared for analysis in the same manner as the as-received solids. Small portions of the dried solids were also submitted for analysis by XRD. 
SRNL-STI-2012-00309

Revision 0

Table 2-1. Sampling Locations of Tank 16H Annulus Samples

\begin{tabular}{|l|c|c|c|}
\hline Sample ID & Sample Location & Date Received & $\begin{array}{c}\text { Total Weight of } \\
\text { Sample Received }\end{array}$ \\
\hline HTF-16-N-1 & N (North) & $11 / 14 / 2011$ & $162.1 \mathrm{~g}$ \\
\hline HTF-16-S-1 & S (South)* & $9 / 13 / 2011$ & - \\
\hline HTF-16-E-1 & E (East)* & $10 / 3 / 2011$ & - \\
\hline HTF-16-W-1 & W (West) & $12 / 1 / 2011$ & $234.8 \mathrm{~g}$ \\
\hline HTF-16-S-2 & S (South) & $11 / 21 / 2011$ & $208.8 \mathrm{~g}$ \\
\hline HTF-16-E-2 & E (East) & $11 / 7 / 2011$ & $144.4 \mathrm{~g}$ \\
\hline
\end{tabular}

* An insufficient quantity of material was obtained in the initial samples from the South and East risers, so additional samples were obtained from each of these locations.

Table 2-2. Composition of the Analytical Reference Glass-1 Standard.

\begin{tabular}{|l|c|}
\hline Element & $\mathbf{~ m g / k g}$ in Glass \\
\hline $\mathrm{Al}$ & $2.50 \mathrm{E}+04$ \\
\hline $\mathrm{B}$ & $2.69 \mathrm{E}+04$ \\
\hline $\mathrm{Ba}$ & $7.88 \mathrm{E}+02$ \\
\hline $\mathrm{Ca}$ & $1.02 \mathrm{E}+04$ \\
\hline $\mathrm{Cr}$ & $6.36 \mathrm{E}+02$ \\
\hline $\mathrm{Cu}$ & $3.20 \mathrm{E}+01$ \\
\hline $\mathrm{Fe}$ & $9.79 \mathrm{E}+04$ \\
\hline $\mathrm{K}$ & $2.25 \mathrm{E}+04$ \\
\hline $\mathrm{Li}$ & $1.49 \mathrm{E}+04$ \\
\hline $\mathrm{Mg}$ & $5.19 \mathrm{E}+03$ \\
\hline
\end{tabular}

\begin{tabular}{|l|c|}
\hline Element & $\mathbf{m g} / \mathbf{k g}$ in Glass \\
\hline $\mathrm{Mn}$ & $1.46 \mathrm{E}+04$ \\
\hline $\mathrm{Na}$ & $8.53 \mathrm{E}+04$ \\
\hline $\mathrm{Ni}$ & $8.25 \mathrm{E}+03$ \\
\hline $\mathrm{P}$ & $9.60 \mathrm{E}+02$ \\
\hline $\mathrm{Si}$ & $2.24 \mathrm{E}+05$ \\
\hline $\mathrm{Sr}$ & $3.13 \mathrm{E}+01$ \\
\hline $\mathrm{Ti}$ & $6.89 \mathrm{E}+03$ \\
\hline $\mathrm{Zn}$ & $1.61 \mathrm{E}+02$ \\
\hline $\mathrm{Zr}$ & $9.63 \mathrm{E}+02$ \\
\hline
\end{tabular}


SRNL-STI-2012-00309

Revision 0

\subsection{Analytical Results and Discussion}

\subsection{General Information}

Tables 3-2 through 3-13 provide analytical results on the chemical composition of the water leached solids, the oxalic acid leachates, and oxalic acid leached solids from the leaching tests. Figures 3-1 through 3-5 provide photographs of the remaining solids from the oxalic acid leaching test. Table 3-14 and Figures 3-6 through 3-13 provide the results of the XRD analysis of the oxalic acid leached solids. Analytical results for the digested solids use units of $\mathrm{mg} / \mathrm{kg}$ and $\mathrm{dpm} / \mathrm{g}$ of dried solids. Analytical results for leachate solutions use units of $\mathrm{mg} / \mathrm{L}$ and $\mathrm{dpm} / \mathrm{mL}$. Values in italics were not used in the calculation of the average due to the unreliability of the values. The unreliability may be due to the presence of the analyte in the blank/standard or the unsuitability of the digestion method for that analyte. Additionally, the detection limits for the peroxide fusion data in the ICP-ES were generally much higher than for the aqua regia digestion and were not included in determining the less than value for the analyte. The results of the reagent blanks and glass standards prepared concurrently with the sample preparations can be found in the appendix.

For waste tank sample characterization, an uncertainty of approximately $+/-15 \%$ has been found to be the normal range for the combined sampling and analytical uncertainty. ${ }^{8}$ For the Tank $16 \mathrm{H}$ samples, the percent relative standard deviation (\%RSD) presented in the tables only includes the uncertainty associated with sub-sampling in the Shielded Cells and the uncertainty of the analytical method. It should be noted that the samples represent a small amount of material from four small areas in the tank annulus.

The column headings in the data tables indicate the dissolution method used to dissolve the samples ("Aqua Regia" and "Per Fusion"). The data tables also indicate the analytical method used to measure each analyte. Table 3-1 shows the abbreviations used for each analytical method: 
SRNL-STI-2012-00309

Revision 0

Table 3-1. Abbreviations for Analytical Methods used in Data Tables

\begin{tabular}{|l|c|}
\hline Analytical Method & $\begin{array}{c}\text { Abbreviation } \\
\text { in Tables }\end{array}$ \\
\hline Inductively Coupled Plasma-Emission Spectroscopy & IE \\
\hline Inductively Coupled Plasma-Mass Spectrometry & IM \\
\hline Ion Chromatography & IC \\
\hline Titration for Free Hydroxide & TH \\
\hline Separation/Alpha Spectroscopy & SA \\
\hline Gamma Spectroscopy & GS \\
\hline Beta Spectroscopy & BS \\
\hline
\end{tabular}

\subsection{Water Leached Solids Results}

Table 3-2 through 3-5 shows the analytical results of the water leached solids for the four samples. The results show $\mathrm{Si}, \mathrm{Na}, \mathrm{Al}$, and Fe remain as the most abundant elements in all four samples after water leaching. The heterogeneous nature of the material and difficulties in sub-sampling the solids may contribute to the higher \%RSD's found in some of the results. For both digestion methods, the results of the glass standards show close agreement with the expected concentrations and the reagent blanks show no significant contamination (see Table A-1).

The results from the XRD analysis of the water leached solids were previously reported. ${ }^{3}$ The XRD results largely agree with the chemical analysis showing the predominant crystalline mineral phase is either quartz $\left(\mathrm{SiO}_{2}\right)$ or sodium aluminum nitrate silicate hydrate $\left(\mathrm{Na}_{8}\left(\mathrm{Al}_{6} \mathrm{Si}_{6} \mathrm{O}_{24}\right)\left(\mathrm{NO}_{3}\right)_{2} \cdot 4 \mathrm{H}_{2} \mathrm{O}\right)$ in all four samples.

Based on the weight of solids remaining, the water leaching test dissolved $20-35 \%$ of the solids after three contacts with an approximately $3: 1$ volume of water at $45{ }^{\circ} \mathrm{C}$. The chemical analysis of the leachates, along with the XRD results and analysis of the remaining solids, indicate sodium salts of nitrate, nitrite, sulfate, and possibly carbonate/bicarbonate make up the majority of the dissolved material. Based on the results of the leachate analysis, the majority of these salts were dissolved in the first water contact and simply diluted with each subsequent water contact. ${ }^{3}$

\subsection{Oxalic Acid Leachate Results}

Tables 3-6 through 3-9 provide the chemical composition of the decanted leachates from the oxalic acid leaching test. All of the decanted leachates indicate the presence of 
soluble sodium salts of $\mathrm{NO}_{2}{ }^{-}$and $\mathrm{NO}_{3}{ }^{-}$as the species of highest concentration with substantial concentrations of aluminum.

The tables also show two columns labeled "Decant 2/Decant 1" and "Decant 3/Decant 2". The values in the columns represent the fraction from dividing the concentrations in the labeled decant solutions by one another. These columns provide an indication of how material is dissolving from the solids during each oxalic acid contact in the leaching test. Since the leaching test decanted the first contact solution to approximately $50 \mathrm{~mL}$ and then added an additional $150 \mathrm{~mL}$ of oxalic acid for the next contact, simple dilution should give a factor of 0.25 (i.e., $50 /(50+150)=0.25)$ for the ratio of concentrations between contacts. In other words, if all of the material that will be dissolved was dissolved in the first oxalic acid contact, the addition of oxalic acid in second contact merely serves to dilute the concentrations of species in solution from the first contact. In that case, the ratio of the concentration in the second oxalic acid contact to the concentration in the first contact should be $\sim 0.25$. However, since the solutions were decanted to the level of the settled solids during the leaching test, sometimes less and sometimes more than $50 \mathrm{~mL}$ of solution was left behind. Therefore, assuming the volume left after decanting was between $35-65 \mathrm{~mL}$, a ratio of between $\sim 0.2$ to $\sim 0.3$ indicates simple dilution between oxalic acid contacts. A value larger than $\sim 0.3$ indicates that material is still dissolving between each solution contact. A value smaller than $\sim 0.2$ for the ratio of concentrations between oxalic acid contacts may indicate precipitation of the analyte from solution. The values in the tables are nearly all above 0.3 indicating continued dissolution of the analyte with each oxalic acid contact.

Completing a clean separation of the liquid from the solids during the oxalic acid leaching tests proved difficult due to the presence of fine particulates that did not settle within a 24 hour period. One of the samples (HTF-16-S-2) showed the formation of a gel in the bottom of the bottle on the first oxalic acid contact. All of the samples showed gel formation in the $2^{\text {nd }}$ and $3^{\text {rd }}$ oxalic acid contacts. All of the decanted solutions required filtration to remove the fines. The amount of material removed by filtration was miniscule, barely coating the surface of the filter. Figures 3-1 through 3-5 show the appearance of the solids after oxalic acid leaching (beaker dimensions, $37 / 8$ " tall, 2 " wide at base, $2 \frac{1 / 4}{4}$ wide at top). The solids were difficult to filter and formed gummy clumps on the filter. After drying, the solids appear swollen relative to the water leached solids and formed large clumps.

\subsection{Oxalic Acid Leached Solids Results}

Tables 3-10 through 3-13 show the analytical results of the oxalic acid leached solids for the four samples. The results show $\mathrm{Si}, \mathrm{Na}, \mathrm{Al}$, and Fe remain as the most abundant elements in all four samples after leaching. The heterogeneous nature of the material and difficulties in sub-sampling the solids may contribute to the higher \%RSD's found in some of the results. For both digestion methods the results of the glass standards show close agreement with the expected concentrations and the reagent blanks show little contamination (see Table A-2). 
Table 3-14 shows a summary of the results from the XRD analysis and the estimated percentages of each crystalline mineral phase present in the samples. Figures 3-6 through 3-13 show the individual spectra for each sample in duplicate. All four samples after oxalic acid leaching show the presence of quartz $\left(\mathrm{SiO}_{2}\right)$ and/or sodium aluminum nitrate silicate hydrate $\left(\mathrm{Na}_{8}\left(\mathrm{Al}_{6} \mathrm{Si}_{6} \mathrm{O}_{24}\right)\left(\mathrm{NO}_{3}\right)_{2} \cdot 4 \mathrm{H}_{2} \mathrm{O}\right)$ crystalline phases except for HTF-16S2 that still contains some gibbsite $\left(\mathrm{Al}(\mathrm{OH})_{3}\right)$. These results agree well with the chemical analysis of the remaining solids after oxalic acid leaching. 
SRNL-STI-2012-00309

Revision 0

Table 3-2. Composition of the Water Leached Solids from Tank 16H Annulus Sample HTF-16-E-2

\begin{tabular}{|c|c|c|c|c|c|c|}
\hline Analyte (Method) & $\begin{array}{c}\text { Aqua Regia } \\
\text { Replicate } 1 \\
\text { (mg/kg) }\end{array}$ & $\begin{array}{c}\text { Aqua Regia } \\
\text { Replicate } 2 \\
\text { (mg/kg) }\end{array}$ & $\begin{array}{c}\text { Per Fusion } \\
\text { Replicate } 1 \\
\text { (mg/kg) }\end{array}$ & $\begin{array}{c}\text { Per Fusion } \\
\text { Replicate } 2 \\
\text { (mg/kg) }\end{array}$ & $\begin{array}{c}\text { Average } \\
\text { (mg/kg) }\end{array}$ & $\%$ RSD \\
\hline $\mathrm{Ag}$ (IE) & $<1.03 \mathrm{E}+02$ & $<1.03 \mathrm{E}+02$ & $<9.97 E+02$ & $<1.04 E+03$ & $<1.03 \mathrm{E}+02$ & - \\
\hline $\mathrm{Al}$ (IE) & $8.21 \mathrm{E}+04$ & $1.05 \mathrm{E}+05$ & $9.79 \mathrm{E}+04$ & $1.04 \mathrm{E}+05$ & $9.73 \mathrm{E}+04$ & $11 \%$ \\
\hline B (IE) & $<1.45 \mathrm{E}+01$ & $<1.45 \mathrm{E}+01$ & $<1.40 E+02$ & $<1.46 E+02$ & $<1.45 \mathrm{E}+01$ & - \\
\hline $\mathrm{Ba}$ (IE) & $6.73 \mathrm{E}+01$ & $7.18 \mathrm{E}+01$ & $7.82 \mathrm{E}+01$ & $8.48 \mathrm{E}+01$ & $7.55 \mathrm{E}+01$ & $10 \%$ \\
\hline Be (IE) & $<4.30 \mathrm{E}-01$ & $<4.30 \mathrm{E}-01$ & $<5.54 E+00$ & $<5.80 E+00$ & $<4.30 \mathrm{E}-01$ & - \\
\hline $\mathrm{Ca}$ (IE) & $1.70 \mathrm{E}+04$ & $1.44 \mathrm{E}+04$ & $1.75 E+04$ & $1.61 E+04$ & $1.57 \mathrm{E}+04$ & $12 \%$ \\
\hline $\mathrm{Cd}$ (IE) & $3.80 \mathrm{E}+00$ & $3.51 \mathrm{E}+00$ & $<2.15 E+01$ & $<2.25 E+01$ & $3.66 \mathrm{E}+00$ & $5.6 \%$ \\
\hline $\mathrm{Ce}$ (IE) & $5.93 \mathrm{E}+01$ & $5.97 \mathrm{E}+01$ & $<4.89 E+02$ & $<5.12 E+02$ & $5.95 \mathrm{E}+01$ & $0.5 \%$ \\
\hline Co (IE) & $<6.95 \mathrm{E}+00$ & $<6.95 \mathrm{E}+00$ & $<6.71 E+01$ & $<7.03 E+01$ & $<6.95 \mathrm{E}+00$ & - \\
\hline $\mathrm{Cr}$ (IE) & $4.51 \mathrm{E}+02$ & $4.34 \mathrm{E}+02$ & $5.05 \mathrm{E}+02$ & $6.83 \mathrm{E}+02$ & $5.18 \mathrm{E}+02$ & $22 \%$ \\
\hline $\mathrm{Cu}$ (IE) & $1.36 \mathrm{E}+02$ & $1.24 \mathrm{E}+02$ & $1.43 \mathrm{E}+02$ & $1.53 \mathrm{E}+02$ & $1.39 \mathrm{E}+02$ & $8.8 \%$ \\
\hline $\mathrm{Fe}(\mathrm{IE})$ & $4.59 \mathrm{E}+04$ & $2.56 \mathrm{E}+04$ & $4.36 \mathrm{E}+04$ & $3.20 \mathrm{E}+04$ & $3.68 \mathrm{E}+04$ & $26 \%$ \\
\hline Gd (IE) & $<1.54 \mathrm{E}+01$ & $<1.54 \mathrm{E}+01$ & $<1.49 E+02$ & $<1.56 E+02$ & $<1.54 \mathrm{E}+01$ & - \\
\hline K (IE) & $1.28 \mathrm{E}+03$ & $1.54 \mathrm{E}+03$ & $2.91 E+03$ & $4.20 E+03$ & $1.41 \mathrm{E}+03$ & $13 \%$ \\
\hline La (IE) & $2.42 \mathrm{E}+01$ & $2.78 \mathrm{E}+01$ & $<4.64 E+01$ & $<4.86 E+01$ & $2.60 \mathrm{E}+01$ & $9.8 \%$ \\
\hline Li (IE) & $<1.05 \mathrm{E}+01$ & $<1.05 \mathrm{E}+01$ & $<1.01 E+02$ & $<1.06 E+02$ & $<1.05 \mathrm{E}+01$ & - \\
\hline $\mathrm{Mg}$ (IE) & $1.13 \mathrm{E}+03$ & $9.03 \mathrm{E}+02$ & $1.83 \mathrm{E}+03$ & $1.75 \mathrm{E}+03$ & $1.40 \mathrm{E}+03$ & $33 \%$ \\
\hline $\mathrm{Mn}$ (IE) & $3.31 \mathrm{E}+02$ & $2.38 \mathrm{E}+02$ & $3.56 \mathrm{E}+02$ & $3.35 \mathrm{E}+02$ & $3.15 \mathrm{E}+02$ & $17 \%$ \\
\hline Mo (IE) & $<4.42 \mathrm{E}+01$ & $<4.42 \mathrm{E}+01$ & $<1.85 E+02$ & $<1.94 E+02$ & $<4.42 \mathrm{E}+01$ & - \\
\hline $\mathrm{Na}$ (IE) & $8.50 \mathrm{E}+04$ & $1.05 \mathrm{E}+05$ & - & - & $9.50 \mathrm{E}+04$ & $15 \%$ \\
\hline Ni (IE) & $7.19 \mathrm{E}+01$ & $6.00 \mathrm{E}+01$ & $<3.15 E+02$ & $<3.30 E+02$ & $6.60 \mathrm{E}+01$ & $13 \%$ \\
\hline $\mathrm{P}$ (IE) & $1.08 \mathrm{E}+02$ & $1.04 \mathrm{E}+02$ & $<5.99 E+02$ & $<6.28 E+02$ & $1.06 \mathrm{E}+02$ & $2.7 \%$ \\
\hline $\mathrm{Pb}$ (IE) & $3.53 \mathrm{E}+02$ & $3.71 \mathrm{E}+02$ & $<4.96 E+02$ & $<5.19 E+02$ & $3.62 \mathrm{E}+02$ & $3.5 \%$ \\
\hline $\mathrm{S}$ (IE) & $5.56 \mathrm{E}+02$ & $<5.38 \mathrm{E}+02$ & $<5.19 E+03$ & $<5.43 E+03$ & $5.56 \mathrm{E}+02$ & - \\
\hline $\mathrm{Sb}$ (IE) & $<9.15 \mathrm{E}+01$ & $<9.15 \mathrm{E}+01$ & $<7.38 \mathrm{E}+02$ & $<7.72 \mathrm{E}+02$ & $<9.15 \mathrm{E}+01$ & - \\
\hline Si (IE) & - & - & $2.04 \mathrm{E}+05$ & $2.17 \mathrm{E}+05$ & $2.11 \mathrm{E}+05$ & $4.4 \%$ \\
\hline Sn (IE) & $<4.02 \mathrm{E}+01$ & $<4.02 \mathrm{E}+01$ & $<3.88 E+02$ & $<4.07 E+02$ & $<4.02 \mathrm{E}+01$ & - \\
\hline
\end{tabular}

Values in italics were not used in calculating the average. Divide $\mathrm{mg} / \mathrm{kg}$ values by $1 \mathrm{E}+04$ to convert to wt $\%$ basis. 
Table 3-2. Composition of the Water Leached Solids from Tank 16H Annulus Sample HTF-16-E-2 (continued)

\begin{tabular}{|l|c|c|c|c|c|c||}
\hline Analyte (Method) & $\begin{array}{c}\text { Aqua Regia } \\
\text { Replicate 1 } \\
\text { (mg/kg) }\end{array}$ & $\begin{array}{c}\text { Aqua Regia } \\
\text { Replicate 2 } \\
\text { (mg/kg) }\end{array}$ & $\begin{array}{c}\text { Per Fusion } \\
\text { Replicate 1 } \\
\text { (mg/kg) }\end{array}$ & $\begin{array}{c}\text { Per Fusion } \\
\text { Replicate 2 } \\
\text { (mg/kg) }\end{array}$ & $\begin{array}{c}\text { Average } \\
\text { (mg/kg) }\end{array}$ & \%RSD \\
\hline Sr (IE) & $5.30 \mathrm{E}+01$ & $5.22 \mathrm{E}+01$ & $8.03 E+01$ & $8.15 E+01$ & $5.26 \mathrm{E}+01$ & $1.1 \%$ \\
\hline Th (IE) & $<3.84 \mathrm{E}+01$ & $<3.84 \mathrm{E}+01$ & $<3.79 E+02$ & $<3.97 E+02$ & $<3.84 \mathrm{E}+01$ & - \\
\hline Ti (IE) & $1.05 E+02$ & $8.40 E+01$ & $1.67 \mathrm{E}+02$ & $2.00 \mathrm{E}+02$ & $1.84 \mathrm{E}+02$ & $13 \%$ \\
\hline $\mathrm{U}(\mathrm{IE})$ & $7.24 \mathrm{E}+02$ & $6.21 \mathrm{E}+02$ & $<2.26 E+03$ & $<2.37 \mathrm{E}+03$ & $6.73 \mathrm{E}+02$ & $11 \%$ \\
\hline $\mathrm{V}(\mathrm{IE})$ & $<3.37 \mathrm{E}+00$ & $<3.37 \mathrm{E}+00$ & $<3.25 E+01$ & $<3.41 \mathrm{E}+01$ & $<3.37 \mathrm{E}+00$ & - \\
\hline $\mathrm{Zn}(\mathrm{IE})$ & $7.04 \mathrm{E}+02$ & $7.53 \mathrm{E}+02$ & $8.53 \mathrm{E}+02$ & $1.00 \mathrm{E}+03$ & $8.28 \mathrm{E}+02$ & $16 \%$ \\
\hline $\mathrm{Zr}(\mathrm{IE})$ & $5.22 \mathrm{E}+01$ & $5.71 \mathrm{E}+01$ & - & - & $5.47 \mathrm{E}+01$ & $6.3 \%$ \\
\hline $\mathrm{Hg}(\mathrm{CV})$ & $1.91 \mathrm{E}+03$ & $1.78 \mathrm{E}+03$ & - & - & $1.85 \mathrm{E}+03$ & $5.0 \%$ \\
\hline${ }^{232} \mathrm{Th}(\mathrm{IM})$ & $2.45 \mathrm{E}+00$ & $1.77 \mathrm{E}+00$ & $2.53 \mathrm{E}+00$ & $2.88 \mathrm{E}+00$ & $2.41 \mathrm{E}+00$ & $19 \%$ \\
\hline${ }^{233} \mathrm{U}(\mathrm{IM})$ & $<8.91 \mathrm{E}-01$ & $<8.96 \mathrm{E}-01$ & $<8.65 \mathrm{E}-01$ & $<9.06 \mathrm{E}-01$ & $<8.89 \mathrm{E}-01$ & - \\
\hline${ }^{234} \mathrm{U}(\mathrm{IM})$ & $<8.91 \mathrm{E}-01$ & $<8.96 \mathrm{E}-01$ & $<8.65 \mathrm{E}-01$ & $<9.06 \mathrm{E}-01$ & $<8.89 \mathrm{E}-01$ & - \\
\hline${ }^{235} \mathrm{U}(\mathrm{IM})$ & $5.75 \mathrm{E}+00$ & $6.49 \mathrm{E}+00$ & $7.44 \mathrm{E}+00$ & $6.75 \mathrm{E}+00$ & $6.61 \mathrm{E}+00$ & $11 \%$ \\
\hline${ }^{236} \mathrm{U}(\mathrm{IM})$ & $9.65 \mathrm{E}-01$ & $9.25 \mathrm{E}-01$ & $<1.73 \mathrm{E}+00$ & $<1.81 \mathrm{E}+00$ & $9.45 \mathrm{E}-01$ & $3.0 \%$ \\
\hline${ }^{238} \mathrm{U}(\mathrm{IM})$ & $1.44 \mathrm{E}+02$ & $1.56 \mathrm{E}+02$ & $1.84 \mathrm{E}+02$ & $1.91 \mathrm{E}+02$ & $1.69 \mathrm{E}+02$ & $13 \%$ \\
\hline $\mathrm{U}-\mathrm{total}(\mathrm{IM})$ & $1.50 \mathrm{E}+02$ & $1.63 \mathrm{E}+02$ & $1.91 \mathrm{E}+02$ & $1.98 \mathrm{E}+02$ & $1.76 \mathrm{E}+02$ & $13 \%$ \\
\hline${ }^{90} \mathrm{Sr}(\mathrm{BS})$ & $3.70 \mathrm{E}+00$ & $4.52 \mathrm{E}+00$ & $4.81 \mathrm{E}+00$ & $5.73 \mathrm{E}+00$ & $4.69 \mathrm{E}+00$ & $18 \%$ \\
\hline${ }^{137} \mathrm{Cs}(\mathrm{GS})$ & $<8.91 \mathrm{E}-01$ & $<8.96 \mathrm{E}-01$ & $<1.73 \mathrm{E}+00$ & $<1.81 \mathrm{E}+00$ & $<1.33 \mathrm{E}+00$ & - \\
\hline${ }^{238} \mathrm{Pu}(\mathrm{SA})$ & $3.70 \mathrm{E}+00$ & $4.52 \mathrm{E}+00$ & $4.81 \mathrm{E}+00$ & $5.73 \mathrm{E}+00$ & $4.69 \mathrm{E}+00$ & $18 \%$ \\
\hline${ }^{99} \mathrm{Tc}(\mathrm{IM})$ & $7.97 \mathrm{E}+00$ & $8.78 \mathrm{E}+00$ & $1.01 \mathrm{E}+01$ & $1.11 \mathrm{E}+01$ & $9.48 \mathrm{E}+00$ & $15 \%$ \\
\hline${ }^{237} \mathrm{~Np}(\mathrm{IM})$ & $2.68 \mathrm{E}+00$ & $2.89 \mathrm{E}+00$ & $3.68 \mathrm{E}+00$ & $2.75 \mathrm{E}+00$ & $3.00 \mathrm{E}+00$ & $15 \%$ \\
\hline${ }^{240} \mathrm{Pu}(\mathrm{IM})$ & $3.70 \mathrm{E}+00$ & $4.52 \mathrm{E}+00$ & $4.81 \mathrm{E}+00$ & $5.73 \mathrm{E}+00$ & $4.69 \mathrm{E}+00$ & $18 \%$ \\
\hline$<8.91 \mathrm{E}-01$ & $<8.96 \mathrm{E}-01$ & $<1.73 \mathrm{E}+00$ & $<1.81 \mathrm{E}+00$ & $<1.33 \mathrm{E}+00$ & - \\
\hline
\end{tabular}

Values in italics were not used in calculating the average. Divide $\mathrm{mg} / \mathrm{kg}$ values by $1 \mathrm{E}+04$ to convert to wt $\%$ basis. 
SRNL-STI-2012-00309

Revision 0

Table 3-2. Composition of the Water Leached Solids from Tank 16H Annulus Sample HTF-16-E-2 (continued)

\begin{tabular}{|c|c|c|c|c|c|c|}
\hline Analyte (Method) & $\begin{array}{c}\text { Aqua Regia } \\
\text { Replicate } 1 \\
\text { (dpm/g) } \\
\end{array}$ & $\begin{array}{c}\text { Aqua Regia } \\
\text { Replicate } 2 \\
\text { (dpm/g) }\end{array}$ & $\begin{array}{c}\text { Per Fusion } \\
\text { Replicate } 1 \\
\text { (dpm/g) }\end{array}$ & $\begin{array}{c}\text { Per Fusion } \\
\text { Replicate } 2 \\
\text { (dpm/g) }\end{array}$ & $\begin{array}{l}\text { Average } \\
(\mathrm{dpm} / \mathrm{g})\end{array}$ & $\%$ RSD \\
\hline${ }^{90} \mathrm{Sr}(\mathrm{BS})$ & $1.29 \mathrm{E}+09$ & $1.40 \mathrm{E}+09$ & $1.27 \mathrm{E}+09$ & $1.55 \mathrm{E}+09$ & $1.38 \mathrm{E}+09$ & $9.3 \%$ \\
\hline${ }^{137} \mathrm{Cs}$ (GS) & $2.22 \mathrm{E}+09$ & $2.45 \mathrm{E}+09$ & $2.72 \mathrm{E}+09$ & $2.52 \mathrm{E}+09$ & $2.48 \mathrm{E}+09$ & $8.3 \%$ \\
\hline${ }^{238} \mathrm{Pu}(\mathrm{SA})$ & $4.57 \mathrm{E}+06$ & $4.81 \mathrm{E}+06$ & $4.70 \mathrm{E}+06$ & $6.16 \mathrm{E}+06$ & $5.06 \mathrm{E}+06$ & $15 \%$ \\
\hline${ }^{239 / 240} \mathrm{Pu}(\mathrm{SA})$ & $8.08 \mathrm{E}+05$ & $7.81 \mathrm{E}+05$ & $7.96 \mathrm{E}+05$ & $1.08 \mathrm{E}+06$ & $8.66 \mathrm{E}+05$ & $16 \%$ \\
\hline${ }^{99}$ Tc (IM) & $3.00 \mathrm{E}+05$ & $3.31 \mathrm{E}+05$ & $3.79 \mathrm{E}+05$ & $4.19 \mathrm{E}+05$ & $3.57 \mathrm{E}+05$ & $15 \%$ \\
\hline${ }^{232} \mathrm{Th}$ (IM) & $5.99 \mathrm{E}-01$ & 4.32E-01 & $6.17 \mathrm{E}-01$ & $7.04 \mathrm{E}-01$ & $5.88 \mathrm{E}-01$ & $19 \%$ \\
\hline${ }^{233} \mathrm{U}(\mathrm{IM})$ & $<1.91 \mathrm{E}+04$ & $<1.92 \mathrm{E}+04$ & $<1.85 \mathrm{E}+04$ & $<1.94 \mathrm{E}+04$ & $<1.90 \mathrm{E}+04$ & - \\
\hline${ }^{234} \mathrm{U}$ (IM) & $<1.24 \mathrm{E}+04$ & $<1.25 \mathrm{E}+04$ & $<1.20 \mathrm{E}+04$ & $<1.26 \mathrm{E}+04$ & $<1.24 \mathrm{E}+04$ & - \\
\hline${ }^{235} \mathrm{U}(\mathrm{IM})$ & $2.76 \mathrm{E}+01$ & $3.11 \mathrm{E}+01$ & $3.57 \mathrm{E}+01$ & $3.24 \mathrm{E}+01$ & $3.17 \mathrm{E}+01$ & $11 \%$ \\
\hline${ }^{236} \mathrm{U}$ (IM) & $1.39 \mathrm{E}+02$ & $1.33 \mathrm{E}+02$ & $<2.48 \mathrm{E}+02$ & $<2.60 \mathrm{E}+02$ & $1.36 \mathrm{E}+02$ & $3.0 \%$ \\
\hline${ }^{237} \mathrm{~Np}$ (IM) & $4.20 \mathrm{E}+03$ & $4.51 \mathrm{E}+03$ & $5.75 \mathrm{E}+03$ & $4.30 \mathrm{E}+03$ & $4.69 \mathrm{E}+03$ & $15 \%$ \\
\hline${ }^{238} \mathrm{U}(\mathrm{IM})$ & $1.07 \mathrm{E}+02$ & $1.16 \mathrm{E}+02$ & $1.37 \mathrm{E}+02$ & $1.43 \mathrm{E}+02$ & $1.26 \mathrm{E}+02$ & $13 \%$ \\
\hline${ }^{239} \mathrm{Pu}$ (IM) & $5.04 \mathrm{E}+05$ & $6.16 \mathrm{E}+05$ & $6.54 \mathrm{E}+05$ & $7.80 \mathrm{E}+05$ & $6.38 \mathrm{E}+05$ & $18 \%$ \\
\hline${ }^{240} \mathrm{Pu}$ (IM) & $<4.46 \mathrm{E}+05$ & $<4.49 \mathrm{E}+05$ & $<8.66 \mathrm{E}+05$ & $<9.07 \mathrm{E}+05$ & $<6.67 \mathrm{E}+05$ & - \\
\hline
\end{tabular}

Values in italics were not used in calculating the average. Divide $\mathrm{mg} / \mathrm{kg}$ values by $1 \mathrm{E}+04$ to convert to wt $\%$ basis. 
SRNL-STI-2012-00309

Revision 0

Table 3-3. Composition of the Water Leached Solids from Tank 16H Annulus Sample HTF-16-N-1

\begin{tabular}{|c|c|c|c|c|c|c|}
\hline Analyte (Method) & $\begin{array}{c}\text { Aqua Regia } \\
\text { Replicate } 1 \\
\text { (mg/kg) }\end{array}$ & $\begin{array}{c}\text { Aqua Regia } \\
\text { Replicate } 2 \\
\text { (mg/kg) }\end{array}$ & $\begin{array}{c}\text { Per Fusion } \\
\text { Replicate } 1 \\
\text { (mg/kg) }\end{array}$ & $\begin{array}{c}\text { Per Fusion } \\
\text { Replicate } 2 \\
\text { (mg/kg) }\end{array}$ & $\begin{array}{c}\text { Average } \\
(\mathbf{m g} / \mathbf{k g})\end{array}$ & $\%$ RSD \\
\hline $\mathrm{Ag}$ (IE) & $<1.03 \mathrm{E}+02$ & $<1.07 \mathrm{E}+02$ & $<1.10 E+03$ & $<1.10 E+03$ & $<1.05 \mathrm{E}+02$ & - \\
\hline $\mathrm{Al}$ (IE) & $1.13 \mathrm{E}+05$ & $1.08 \mathrm{E}+05$ & $9.86 \mathrm{E}+04$ & $5.90 \mathrm{E}+04$ & $9.47 \mathrm{E}+04$ & $26 \%$ \\
\hline B (IE) & $<1.45 \mathrm{E}+01$ & $<1.50 \mathrm{E}+01$ & $<1.55 E+02$ & $<1.55 E+02$ & $<1.48 \mathrm{E}+01$ & - \\
\hline $\mathrm{Ba}$ (IE) & $4.81 \mathrm{E}+01$ & $3.10 \mathrm{E}+01$ & $<3.98 E+01$ & $<3.98 E+01$ & $3.96 \mathrm{E}+01$ & $31 \%$ \\
\hline $\mathrm{Be}$ (IE) & $<4.30 \mathrm{E}-01$ & $<4.46 \mathrm{E}-01$ & $<6.13 E+00$ & $<6.13 E+00$ & $<4.38 \mathrm{E}-01$ & - \\
\hline $\mathrm{Ca}$ (IE) & $2.70 \mathrm{E}+03$ & $1.63 \mathrm{E}+03$ & $5.83 E+03$ & $3.33 E+03$ & $2.17 \mathrm{E}+03$ & $35 \%$ \\
\hline $\mathrm{Cd}$ (IE) & $<2.22 \mathrm{E}+00$ & $<2.30 \mathrm{E}+00$ & $<2.38 E+01$ & $<2.38 E+01$ & $<2.26 \mathrm{E}+00$ & - \\
\hline $\mathrm{Ce}$ (IE) & $<5.07 \mathrm{E}+01$ & $<5.26 \mathrm{E}+01$ & $<5.42 E+02$ & $<5.42 E+02$ & $<5.17 \mathrm{E}+01$ & - \\
\hline Co (IE) & $<6.95 \mathrm{E}+00$ & $<7.21 \mathrm{E}+00$ & $<7.43 E+01$ & $<7.43 E+01$ & $<7.08 \mathrm{E}+00$ & - \\
\hline Cr (IE) & $5.89 \mathrm{E}+01$ & $4.39 \mathrm{E}+01$ & $9.66 \mathrm{E}+01$ & $8.70 \mathrm{E}+01$ & $7.16 \mathrm{E}+01$ & $34 \%$ \\
\hline $\mathrm{Cu}$ (IE) & $1.11 \mathrm{E}+02$ & $8.89 \mathrm{E}+01$ & $1.27 \mathrm{E}+02$ & $7.89 \mathrm{E}+01$ & $1.01 \mathrm{E}+02$ & $21 \%$ \\
\hline $\mathrm{Fe}(\mathrm{IE})$ & $7.32 \mathrm{E}+03$ & $9.87 \mathrm{E}+03$ & $1.31 \mathrm{E}+04$ & $1.09 \mathrm{E}+03$ & $7.85 \mathrm{E}+03$ & $65 \%$ \\
\hline Gd (IE) & $<1.54 \mathrm{E}+01$ & $<1.60 \mathrm{E}+01$ & $<1.65 E+02$ & $<1.65 E+02$ & $<1.57 \mathrm{E}+01$ & - \\
\hline $\mathrm{K}$ (IE) & $4.69 \mathrm{E}+02$ & $2.80 \mathrm{E}+02$ & $3.84 E+03$ & $3.14 E+03$ & $3.75 \mathrm{E}+02$ & $36 \%$ \\
\hline La (IE) & $1.34 \mathrm{E}+01$ & $7.06 \mathrm{E}+00$ & $<5.13 E+01$ & $<5.13 E+01$ & $1.02 \mathrm{E}+01$ & $44 \%$ \\
\hline Li (IE) & $<1.05 \mathrm{E}+01$ & $<1.09 \mathrm{E}+01$ & $<1.12 E+02$ & $<1.12 E+02$ & $<1.07 \mathrm{E}+01$ & - \\
\hline $\mathrm{Mg}$ (IE) & $7.12 \mathrm{E}+02$ & $1.17 \mathrm{E}+02$ & $5.15 \mathrm{E}+02$ & $5.52 \mathrm{E}+02$ & $4.74 \mathrm{E}+02$ & $53 \%$ \\
\hline Mn (IE) & $7.89 \mathrm{E}+01$ & $7.22 \mathrm{E}+01$ & $1.03 \mathrm{E}+02$ & $<1.61 E+01$ & $8.47 \mathrm{E}+01$ & $19 \%$ \\
\hline Mo (IE) & $<4.42 \mathrm{E}+01$ & $<4.59 \mathrm{E}+01$ & $<2.05 E+02$ & $<2.05 E+02$ & $<4.51 \mathrm{E}+01$ & - \\
\hline $\mathrm{Na}$ (IE) & $1.11 \mathrm{E}+05$ & $1.05 \mathrm{E}+05$ & - & - & $1.08 \mathrm{E}+05$ & $3.9 \%$ \\
\hline $\mathrm{Ni}$ (IE) & $<3.26 \mathrm{E}+01$ & $<3.38 \mathrm{E}+01$ & $<3.49 E+02$ & $<3.49 E+02$ & $<3.32 \mathrm{E}+01$ & - \\
\hline $\mathrm{P}$ (IE) & $<6.21 \mathrm{E}+01$ & $<6.44 \mathrm{E}+01$ & $<6.64 E+02$ & $<6.64 E+02$ & $<6.33 \mathrm{E}+01$ & - \\
\hline $\mathrm{Pb}$ (IE) & $3.76 \mathrm{E}+02$ & $2.55 \mathrm{E}+02$ & $<5.49 E+02$ & $<5.49 E+02$ & $3.16 \mathrm{E}+02$ & $27 \%$ \\
\hline S (IE) & $<5.38 \mathrm{E}+02$ & $<5.58 \mathrm{E}+02$ & $<5.75 E+03$ & $<5.75 E+03$ & $<5.48 \mathrm{E}+02$ & - \\
\hline $\mathrm{Sb}$ (IE) & $<9.15 \mathrm{E}+01$ & $<9.49 \mathrm{E}+01$ & $<8.17 E+02$ & $<8.17 E+02$ & $<9.32 \mathrm{E}+01$ & - \\
\hline Si (IE) & - & - & $2.62 \mathrm{E}+05$ & $2.39 \mathrm{E}+05$ & $2.51 \mathrm{E}+05$ & $6.5 \%$ \\
\hline Sn (IE) & $<4.02 \mathrm{E}+01$ & $<4.17 \mathrm{E}+01$ & $<4.30 E+02$ & $<4.30 E+02$ & $<4.10 \mathrm{E}+01$ & - \\
\hline
\end{tabular}

Values in italics were not used in calculating the average. Divide $\mathrm{mg} / \mathrm{kg}$ values by $1 \mathrm{E}+04$ to convert to wt $\%$ basis. 
Table 3-3. Composition of the Water Leached Solids from Tank 16H Annulus Sample HTF-16-N-1 (continued)

\begin{tabular}{|l|c|c|c|c|c|c||}
\hline Analyte (Method) & $\begin{array}{c}\text { Aqua Regia } \\
\text { Replicate 1 } \\
\text { (mg/kg) }\end{array}$ & $\begin{array}{c}\text { Aqua Regia } \\
\text { Replicate 2 } \\
\text { (mg/kg) }\end{array}$ & $\begin{array}{c}\text { Per Fusion } \\
\text { Replicate 1 } \\
\text { (mg/kg) }\end{array}$ & $\begin{array}{c}\text { Per Fusion } \\
\text { Replicate 2 } \\
\text { (mg/kg) }\end{array}$ & $\begin{array}{c}\text { Average } \\
\text { (mg/kg) }\end{array}$ & \%RSD \\
\hline Sr (IE) & $9.96 \mathrm{E}+00$ & $6.84 \mathrm{E}+00$ & $3.99 E+01$ & $3.38 E+01$ & $8.40 \mathrm{E}+00$ & $26 \%$ \\
\hline Th (IE) & $<3.84 \mathrm{E}+01$ & $<3.98 \mathrm{E}+01$ & $<4.20 E+02$ & $<4.20 E+02$ & $<3.91 \mathrm{E}+01$ & - \\
\hline Ti (IE) & $9.41 E+01$ & $3.29 E+01$ & $2.95 \mathrm{E}+02$ & $3.15 \mathrm{E}+02$ & $3.05 \mathrm{E}+02$ & $4.6 \%$ \\
\hline $\mathrm{U}(\mathrm{IE})$ & $<2.34 \mathrm{E}+02$ & $<2.43 \mathrm{E}+02$ & $<2.50 E+03$ & $<2.50 E+03$ & $<2.39 \mathrm{E}+02$ & - \\
\hline $\mathrm{V}(\mathrm{IE})$ & $6.59 \mathrm{E}+00$ & $<3.49 \mathrm{E}+00$ & $<3.60 \mathrm{E}+01$ & $<3.60 \mathrm{E}+01$ & $6.59 \mathrm{E}+00$ & - \\
\hline $\mathrm{Zn}(\mathrm{IE})$ & $6.89 \mathrm{E}+02$ & $6.98 \mathrm{E}+02$ & $7.74 \mathrm{E}+02$ & $1.65 \mathrm{E}+03$ & $9.53 \mathrm{E}+02$ & $49 \%$ \\
\hline $\mathrm{Zr}(\mathrm{IE})$ & $3.56 \mathrm{E}+01$ & $1.46 \mathrm{E}+01$ & - & - & $2.51 \mathrm{E}+01$ & $59 \%$ \\
\hline $\mathrm{Hg}(\mathrm{CV})$ & $1.03 \mathrm{E}+03$ & $4.27 \mathrm{E}+02$ & - & - & $7.29 \mathrm{E}+02$ & $59 \%$ \\
\hline${ }^{232} \mathrm{Th}(\mathrm{IM})$ & $1.39 \mathrm{E}+00$ & $9.29 \mathrm{E}-01$ & $1.71 \mathrm{E}+00$ & $1.53 \mathrm{E}+00$ & $1.39 \mathrm{E}+00$ & $24 \%$ \\
\hline${ }^{233} \mathrm{U}(\mathrm{IM})$ & $<8.96 \mathrm{E}-01$ & $<9.29 \mathrm{E}-01$ & $<9.58 \mathrm{E}-01$ & $<9.58 \mathrm{E}-01$ & $<9.35 \mathrm{E}-01$ & - \\
\hline${ }^{234} \mathrm{U}(\mathrm{IM})$ & $<8.96 \mathrm{E}-01$ & $<9.29 \mathrm{E}-01$ & $<9.58 \mathrm{E}-01$ & $<9.58 \mathrm{E}-01$ & $<9.35 \mathrm{E}-01$ & - \\
\hline${ }^{235} \mathrm{U}(\mathrm{IM})$ & $2.50 \mathrm{E}+00$ & $<1.39 \mathrm{E}+00$ & $1.44 \mathrm{E}+00$ & $1.79 \mathrm{E}+00$ & $1.91 \mathrm{E}+00$ & $28 \%$ \\
\hline${ }^{236} \mathrm{U}(\mathrm{IM})$ & $<8.96 \mathrm{E}-01$ & $<9.29 \mathrm{E}-01$ & $<1.92 \mathrm{E}+00$ & $<1.92 \mathrm{E}+00$ & $<1.41 \mathrm{E}+00$ & - \\
\hline${ }^{238} \mathrm{U}(\mathrm{IM})$ & $4.88 \mathrm{E}+01$ & $2.04 \mathrm{E}+01$ & $1.49 \mathrm{E}+01$ & $2.96 \mathrm{E}+01$ & $2.84 \mathrm{E}+01$ & $52 \%$ \\
\hline $\mathrm{U}-\mathrm{total}(\mathrm{IM})$ & $5.13 \mathrm{E}+01$ & $2.18 \mathrm{E}+01$ & $1.63 \mathrm{E}+01$ & $3.13 \mathrm{E}+01$ & $3.02 \mathrm{E}+01$ & $51 \%$ \\
\hline${ }^{90} \mathrm{Sr}(\mathrm{BS})$ & $1.47 \mathrm{E}+00$ & $7.98 \mathrm{E}-01$ & $9.20 \mathrm{E}-01$ & $3.14 \mathrm{E}-01$ & $8.75 \mathrm{E}-01$ & $54 \%$ \\
\hline${ }^{137} \mathrm{Cs}(\mathrm{GS})$ & $1.80 \mathrm{E}+01$ & $1.98 \mathrm{E}+01$ & $1.46 \mathrm{E}+01$ & $1.03 \mathrm{E}+01$ & $1.57 \mathrm{E}+01$ & $27 \%$ \\
\hline${ }^{238} \mathrm{Pu}(\mathrm{SA})$ & $4.89 \mathrm{E}-02$ & $2.50 \mathrm{E}-02$ & $3.03 \mathrm{E}-02$ & $1.35 \mathrm{E}-02$ & $2.94 \mathrm{E}-02$ & $50 \%$ \\
\hline${ }^{99} \mathrm{Tc}(\mathrm{IM})$ & $7.96 \mathrm{E}+00$ & $4.66 \mathrm{E}+00$ & $2.44 \mathrm{E}+00$ & $5.46 \mathrm{E}+00$ & $5.13 \mathrm{E}+00$ & $44 \%$ \\
\hline${ }^{237} \mathrm{~Np}(\mathrm{IM})$ & $<8.96 \mathrm{E}-01$ & $<9.29 \mathrm{E}-01$ & $<2.39 \mathrm{E}+00$ & $<2.39 \mathrm{E}+00$ & $<1.65 \mathrm{E}+00$ & - \\
\hline${ }^{239} \mathrm{Pu}(\mathrm{IM})$ & $1.90 \mathrm{E}+00$ & $<1.86 \mathrm{E}+00$ & $<9.58 \mathrm{E}-01$ & $<9.58 \mathrm{E}-01$ & $<1.42 \mathrm{E}+00$ & - \\
\hline $240 \mathrm{IM})$ & $<8.96 \mathrm{E}-01$ & $<9.29 \mathrm{E}-01$ & $<1.92 \mathrm{E}+00$ & $<1.92 \mathrm{E}+00$ & $<1.41 \mathrm{E}+00$ & - \\
\hline
\end{tabular}

Values in italics were not used in calculating the average. Divide $\mathrm{mg} / \mathrm{kg}$ values by $1 \mathrm{E}+04$ to convert to wt $\%$ basis. 
SRNL-STI-2012-00309

Revision 0

Table 3-3. Composition of the Water Leached Solids from Tank $16 \mathrm{H}$ Annulus Sample HTF-16-N-1 (continued)

\begin{tabular}{|c|c|c|c|c|c|c|}
\hline Analyte (Method) & $\begin{array}{c}\text { Aqua Regia } \\
\text { Replicate } 1 \\
\text { (dpm/g) } \\
\end{array}$ & $\begin{array}{c}\text { Aqua Regia } \\
\text { Replicate } 2 \\
\text { (dpm/g) }\end{array}$ & $\begin{array}{c}\text { Per Fusion } \\
\text { Replicate } 1 \\
\text { (dpm/g) }\end{array}$ & $\begin{array}{c}\text { Per Fusion } \\
\text { Replicate } 2 \\
\text { (dpm/g) }\end{array}$ & $\begin{array}{l}\text { Average } \\
\text { (dpm/g) }\end{array}$ & $\%$ RSD \\
\hline${ }^{90} \mathrm{Sr}(\mathrm{BS})$ & $4.46 \mathrm{E}+08$ & $2.43 \mathrm{E}+08$ & $2.80 \mathrm{E}+08$ & $9.57 \mathrm{E}+07$ & $2.66 \mathrm{E}+08$ & $54 \%$ \\
\hline${ }^{137} \mathrm{Cs}$ (GS) & $3.47 \mathrm{E}+09$ & $3.81 \mathrm{E}+09$ & $2.82 \mathrm{E}+09$ & $1.98 \mathrm{E}+09$ & $3.02 \mathrm{E}+09$ & $27 \%$ \\
\hline${ }^{238} \mathrm{Pu}(\mathrm{SA})$ & $1.86 \mathrm{E}+06$ & $9.52 \mathrm{E}+05$ & $1.15 \mathrm{E}+06$ & $5.14 \mathrm{E}+05$ & $1.12 \mathrm{E}+06$ & $50 \%$ \\
\hline${ }^{239 / 240} \mathrm{Pu}(\mathrm{SA})$ & $3.09 \mathrm{E}+05$ & $1.50 \mathrm{E}+05$ & $1.58 \mathrm{E}+05$ & $7.94 \mathrm{E}+04$ & $1.74 \mathrm{E}+05$ & $55 \%$ \\
\hline${ }^{99}$ Tc (IM) & $3.00 \mathrm{E}+05$ & $1.76 \mathrm{E}+05$ & $9.18 \mathrm{E}+04$ & $2.06 \mathrm{E}+05$ & $1.93 \mathrm{E}+05$ & $44 \%$ \\
\hline${ }^{232} \mathrm{Th}$ (IM) & $3.38 \mathrm{E}-01$ & $2.27 \mathrm{E}-01$ & $4.18 \mathrm{E}-01$ & $3.74 \mathrm{E}-01$ & 3.39E-01 & $24 \%$ \\
\hline${ }^{233} \mathrm{U}(\mathrm{IM})$ & $<1.92 \mathrm{E}+04$ & $<1.99 \mathrm{E}+04$ & $<2.05 \mathrm{E}+04$ & $<2.05 \mathrm{E}+04$ & $<2.00 \mathrm{E}+04$ & - \\
\hline${ }^{234} \mathrm{U}$ (IM) & $<1.25 \mathrm{E}+04$ & $<1.29 \mathrm{E}+04$ & $<1.33 \mathrm{E}+04$ & $<1.33 \mathrm{E}+04$ & $<1.30 \mathrm{E}+04$ & - \\
\hline${ }^{235} \mathrm{U}$ (IM) & $1.20 \mathrm{E}+01$ & $<6.68 \mathrm{E}+00$ & $6.90 \mathrm{E}+00$ & $8.56 \mathrm{E}+00$ & $9.15 \mathrm{E}+00$ & $28 \%$ \\
\hline${ }^{236} \mathrm{U}(\mathrm{IM})$ & $<1.29 \mathrm{E}+02$ & $<1.33 \mathrm{E}+02$ & $<2.75 \mathrm{E}+02$ & $<2.75 \mathrm{E}+02$ & $<2.03 \mathrm{E}+02$ & - \\
\hline${ }^{237} \mathrm{~Np}$ (IM) & $<1.40 \mathrm{E}+03$ & $<1.45 \mathrm{E}+03$ & $<3.75 \mathrm{E}+03$ & $<3.75 \mathrm{E}+03$ & $<2.59 \mathrm{E}+03$ & - \\
\hline${ }^{238} \mathrm{U}(\mathrm{IM})$ & $3.64 \mathrm{E}+01$ & $1.52 \mathrm{E}+01$ & $1.11 \mathrm{E}+01$ & $2.20 \mathrm{E}+01$ & $2.12 \mathrm{E}+01$ & $52 \%$ \\
\hline${ }^{239} \mathrm{Pu}$ (IM) & $2.59 \mathrm{E}+05$ & $<2.53 \mathrm{E}+05$ & $<1.30 \mathrm{E}+05$ & $<1.30 \mathrm{E}+05$ & $<1.93 \mathrm{E}+05$ & - \\
\hline${ }^{240} \mathrm{Pu}$ (IM) & $<4.49 \mathrm{E}+05$ & $<4.65 \mathrm{E}+05$ & $<9.59 \mathrm{E}+05$ & $<9.59 \mathrm{E}+05$ & $<7.08 \mathrm{E}+05$ & - \\
\hline
\end{tabular}

Values in italics were not used in calculating the average. Divide $\mathrm{mg} / \mathrm{kg}$ values by $1 \mathrm{E}+04$ to convert to wt $\%$ basis. 
Table 3-4. Composition of the Water Leached Solids from Tank 16H Annulus Sample HTF-16-S-2

\begin{tabular}{|c|c|c|c|c|c|c|}
\hline Analyte (Method) & $\begin{array}{c}\text { Aqua Regia } \\
\text { Replicate } 1 \\
\text { (mg/kg) }\end{array}$ & $\begin{array}{c}\text { Aqua Regia } \\
\text { Replicate } 2 \\
\text { (mg/kg) }\end{array}$ & $\begin{array}{c}\text { Per Fusion } \\
\text { Replicate } 1 \\
\text { (mg/kg) }\end{array}$ & $\begin{array}{c}\text { Per Fusion } \\
\text { Replicate } 2 \\
\text { (mg/kg) }\end{array}$ & $\begin{array}{c}\text { Average } \\
\text { (mg/kg) }\end{array}$ & $\%$ RSD \\
\hline $\mathrm{Ag}(\mathrm{IE})$ & $<1.11 \mathrm{E}+02$ & $<1.14 \mathrm{E}+02$ & $<1.19 E+03$ & $<1.06 E+03$ & $<1.13 \mathrm{E}+02$ & - \\
\hline $\mathrm{Al}$ (IE) & $7.71 \mathrm{E}+04$ & $8.75 \mathrm{E}+04$ & $9.14 \mathrm{E}+04$ & $6.61 \mathrm{E}+04$ & $8.05 \mathrm{E}+04$ & $14 \%$ \\
\hline B (IE) & $<1.55 \mathrm{E}+01$ & $<1.60 \mathrm{E}+01$ & $<1.66 E+02$ & $<1.49 E+02$ & $<1.58 \mathrm{E}+01$ & - \\
\hline $\mathrm{Ba}$ (IE) & $9.41 \mathrm{E}+01$ & $1.68 \mathrm{E}+02$ & $1.30 \mathrm{E}+02$ & $1.23 \mathrm{E}+02$ & $1.29 \mathrm{E}+02$ & $24 \%$ \\
\hline Be (IE) & $<4.62 \mathrm{E}-01$ & $<4.74 \mathrm{E}-01$ & $<6.58 E+00$ & $<5.90 E+00$ & $<4.68 \mathrm{E}-01$ & - \\
\hline $\mathrm{Ca}$ (IE) & $2.60 \mathrm{E}+03$ & $4.74 \mathrm{E}+03$ & $6.44 E+03$ & $5.87 E+03$ & $3.67 \mathrm{E}+03$ & $41 \%$ \\
\hline Cd (IE) & $4.31 \mathrm{E}+00$ & $6.32 \mathrm{E}+00$ & $<2.55 E+01$ & $<2.29 E+01$ & $5.32 \mathrm{E}+00$ & $27 \%$ \\
\hline $\mathrm{Ce}$ (IE) & $8.20 \mathrm{E}+01$ & $2.08 \mathrm{E}+02$ & $<5.82 E+02$ & $<5.22 E+02$ & $1.45 \mathrm{E}+02$ & $61 \%$ \\
\hline Co (IE) & $8.77 \mathrm{E}+00$ & $1.06 \mathrm{E}+01$ & $<7.98 E+01$ & $<7.16 E+01$ & $9.69 \mathrm{E}+00$ & $13 \%$ \\
\hline $\mathrm{Cr}$ (IE) & $1.77 \mathrm{E}+02$ & $2.70 \mathrm{E}+02$ & $2.98 \mathrm{E}+02$ & $1.71 \mathrm{E}+02$ & $2.29 \mathrm{E}+02$ & $28 \%$ \\
\hline $\mathrm{Cu}$ (IE) & $2.61 \mathrm{E}+02$ & $4.40 \mathrm{E}+02$ & $3.93 \mathrm{E}+02$ & $1.81 \mathrm{E}+02$ & $3.19 \mathrm{E}+02$ & $37 \%$ \\
\hline $\mathrm{Fe}(\mathrm{IE})$ & $3.19 \mathrm{E}+04$ & $2.70 \mathrm{E}+04$ & $2.92 \mathrm{E}+04$ & $2.53 \mathrm{E}+04$ & $2.84 \mathrm{E}+04$ & $10 \%$ \\
\hline Gd (IE) & $<1.65 \mathrm{E}+01$ & $<1.70 \mathrm{E}+01$ & $<1.77 E+02$ & $<1.59 E+02$ & $<1.68 \mathrm{E}+01$ & - \\
\hline $\mathrm{K}$ (IE) & $5.88 \mathrm{E}+02$ & $6.13 \mathrm{E}+02$ & $4.44 E+03$ & $3.75 E+03$ & $6.01 \mathrm{E}+02$ & $2.9 \%$ \\
\hline La (IE) & $3.08 \mathrm{E}+01$ & $8.43 \mathrm{E}+01$ & $5.84 \mathrm{E}+01$ & $<4.94 E+01$ & $5.78 \mathrm{E}+01$ & $46 \%$ \\
\hline Li (IE) & $<1.12 E+01$ & $<1.52 \mathrm{E}+01$ & $<1.20 E+02$ & $<1.08 E+02$ & $<1.52 \mathrm{E}+01$ & - \\
\hline $\mathrm{Mg}$ (IE) & $8.73 E+03$ & $6.94 \mathrm{E}+03$ & $4.30 \mathrm{E}+03$ & $9.00 \mathrm{E}+03$ & $7.24 \mathrm{E}+03$ & $30 \%$ \\
\hline Mn (IE) & $3.16 \mathrm{E}+02$ & $3.05 \mathrm{E}+02$ & $2.80 \mathrm{E}+02$ & $2.14 \mathrm{E}+02$ & $2.79 \mathrm{E}+02$ & $16 \%$ \\
\hline Mo (IE) & $<4.75 \mathrm{E}+01$ & $<4.88 \mathrm{E}+01$ & $<2.21 E+02$ & $<1.98 E+02$ & $<4.82 \mathrm{E}+01$ & - \\
\hline $\mathrm{Na}$ (IE) & $6.57 \mathrm{E}+04$ & $8.06 \mathrm{E}+04$ & - & - & $7.32 \mathrm{E}+04$ & $14 \%$ \\
\hline $\mathrm{Ni}$ (IE) & $1.11 \mathrm{E}+02$ & $1.55 \mathrm{E}+02$ & $<3.74 E+02$ & $<3.36 E+02$ & $1.33 \mathrm{E}+02$ & $23 \%$ \\
\hline $\mathrm{P}$ (IE) & $8.42 \mathrm{E}+01$ & $1.21 \mathrm{E}+02$ & $<7.13 E+02$ & $<6.39 E+02$ & $1.03 \mathrm{E}+02$ & $25 \%$ \\
\hline $\mathrm{Pb}$ (IE) & $8.18 \mathrm{E}+02$ & $7.34 \mathrm{E}+02$ & $8.38 \mathrm{E}+02$ & $2.41 \mathrm{E}+03$ & $1.20 \mathrm{E}+03$ & $67 \%$ \\
\hline S (IE) & $1.20 \mathrm{E}+03$ & $6.70 \mathrm{E}+02$ & $<6.17 E+03$ & $<5.54 E+03$ & $9.35 \mathrm{E}+02$ & $40 \%$ \\
\hline $\mathrm{Sb}$ (IE) & $<9.82 \mathrm{E}+01$ & $<1.01 \mathrm{E}+02$ & $<8.77 E+02$ & $<7.87 E+02$ & $<9.96 \mathrm{E}+01$ & - \\
\hline Si (IE) & - & - & $2.28 \mathrm{E}+05$ & $1.83 \mathrm{E}+05$ & $2.06 \mathrm{E}+05$ & $15 \%$ \\
\hline Sn (IE) & $<4.32 \mathrm{E}+01$ & $<4.43 \mathrm{E}+01$ & $<4.62 E+02$ & $<4.14 E+02$ & $<4.38 \mathrm{E}+01$ & - \\
\hline
\end{tabular}

Values in italics were not used in calculating the average. Divide $\mathrm{mg} / \mathrm{kg}$ values by $1 \mathrm{E}+04$ to convert to wt $\%$ basis. 
SRNL-STI-2012-00309

Revision 0

Table 3-4. Composition of the Water Leached Solids from Tank 16H Annulus Sample HTF-16-S-2 (continued)

\begin{tabular}{|l|c|c|c|c|c|c||}
\hline Analyte (Method) & $\begin{array}{c}\text { Aqua Regia } \\
\text { Replicate 1 } \\
\text { (mg/kg) }\end{array}$ & $\begin{array}{c}\text { Aqua Regia } \\
\text { Replicate 2 } \\
\text { (mg/kg) }\end{array}$ & $\begin{array}{c}\text { Per Fusion } \\
\text { Replicate 1 } \\
\text { (mg/kg) }\end{array}$ & $\begin{array}{c}\text { Per Fusion } \\
\text { Replicate 2 } \\
\text { (mg/kg) }\end{array}$ & $\begin{array}{c}\text { Average } \\
\text { (mg/kg) }\end{array}$ & \%RSD \\
\hline Sr (IE) & $3.62 \mathrm{E}+01$ & $6.96 \mathrm{E}+01$ & $8.56 E+01$ & $6.57 E+01$ & $5.29 \mathrm{E}+01$ & $45 \%$ \\
\hline Th (IE) & $<4.12 \mathrm{E}+01$ & $<4.23 \mathrm{E}+01$ & $<4.51 E+02$ & $<4.04 E+02$ & $<4.18 \mathrm{E}+01$ & - \\
\hline Ti (IE) & $9.54 E+01$ & $1.52 E+02$ & $2.00 \mathrm{E}+02$ & $1.51 \mathrm{E}+02$ & $1.76 \mathrm{E}+02$ & $20 \%$ \\
\hline $\mathrm{U}(\mathrm{IE})$ & $<2.51 E+02$ & $4.72 \mathrm{E}+02$ & $<2.69 E+03$ & $<2.41 E+03$ & $4.72 \mathrm{E}+02$ & - \\
\hline $\mathrm{V}(\mathrm{IE})$ & $3.49 \mathrm{E}+01$ & $1.07 \mathrm{E}+01$ & $<3.87 E+01$ & $<3.47 \mathrm{E}+01$ & $2.28 \mathrm{E}+01$ & $75 \%$ \\
\hline $\mathrm{Zn}(\mathrm{IE})$ & $4.20 \mathrm{E}+02$ & $5.44 \mathrm{E}+02$ & $6.71 \mathrm{E}+02$ & $5.78 \mathrm{E}+02$ & $5.53 \mathrm{E}+02$ & $19 \%$ \\
\hline $\mathrm{Zr}(\mathrm{IE})$ & $9.89 \mathrm{E}+01$ & $2.54 \mathrm{E}+02$ & - & - & $1.76 \mathrm{E}+02$ & $62 \%$ \\
\hline $\mathrm{Hg}(\mathrm{CV})$ & $2.47 \mathrm{E}+03$ & $2.92 \mathrm{E}+03$ & - & - & $2.70 \mathrm{E}+03$ & $12 \%$ \\
\hline${ }^{232} \mathrm{Th}(\mathrm{IM})$ & $1.58 \mathrm{E}+00$ & $3.25 \mathrm{E}+00$ & $2.92 \mathrm{E}+00$ & $2.42 \mathrm{E}+00$ & $2.54 \mathrm{E}+00$ & $29 \%$ \\
\hline${ }^{233} \mathrm{U}(\mathrm{IM})$ & $<9.62 \mathrm{E}-01$ & $<9.88 \mathrm{E}-01$ & $<1.03 \mathrm{E}+00$ & $<9.23 \mathrm{E}-01$ & $<9.75 \mathrm{E}-01$ & - \\
\hline${ }^{234} \mathrm{U}(\mathrm{IM})$ & $<9.62 \mathrm{E}-01$ & $<9.88 \mathrm{E}-01$ & $<1.03 \mathrm{E}+00$ & $<9.23 \mathrm{E}-01$ & $<9.75 \mathrm{E}-01$ & - \\
\hline${ }^{235} \mathrm{U}(\mathrm{IM})$ & $4.33 \mathrm{E}+00$ & $1.05 \mathrm{E}+01$ & $8.76 \mathrm{E}+00$ & $6.61 \mathrm{E}+00$ & $7.55 \mathrm{E}+00$ & $35 \%$ \\
\hline${ }^{236} \mathrm{U}(\mathrm{IM})$ & $<9.62 \mathrm{E}-01$ & $<9.88 \mathrm{E}-01$ & $<2.06 \mathrm{E}+00$ & $<1.85 \mathrm{E}+00$ & $<1.46 \mathrm{E}+00$ & - \\
\hline${ }^{238} \mathrm{U}(\mathrm{IM})$ & $9.51 \mathrm{E}+01$ & $2.57 \mathrm{E}+02$ & $2.24 \mathrm{E}+02$ & $1.53 \mathrm{E}+02$ & $1.82 \mathrm{E}+02$ & $40 \%$ \\
\hline $\mathrm{U}-\mathrm{total}(\mathrm{IM})$ & $9.94 \mathrm{E}+01$ & $2.67 \mathrm{E}+02$ & $2.33 \mathrm{E}+02$ & $1.59 \mathrm{E}+02$ & $1.90 \mathrm{E}+02$ & $40 \%$ \\
\hline${ }^{90} \mathrm{Sr}(\mathrm{BS})$ & $4.76 \mathrm{E}+00$ & $1.06 \mathrm{E}+01$ & $8.31 \mathrm{E}+00$ & $5.88 \mathrm{E}+00$ & $7.39 \mathrm{E}+00$ & $35 \%$ \\
\hline${ }^{137} \mathrm{Cs}(\mathrm{GS})$ & $1.43 \mathrm{E}+01$ & $1.55 \mathrm{E}+01$ & $1.43 \mathrm{E}+01$ & $1.24 \mathrm{E}+01$ & $1.41 \mathrm{E}+01$ & $9.1 \%$ \\
\hline${ }^{238} \mathrm{Pu}(\mathrm{SA})$ & $7.08 \mathrm{E}-02$ & $1.75 \mathrm{E}-01$ & $1.24 \mathrm{E}-01$ & $9.74 \mathrm{E}-02$ & $1.17 \mathrm{E}-01$ & $38 \%$ \\
\hline${ }^{99} \mathrm{Tc}(\mathrm{IM})$ & $1.32 \mathrm{E}+01$ & $1.88 \mathrm{E}+01$ & $2.01 \mathrm{E}+01$ & $1.29 \mathrm{E}+01$ & $1.63 \mathrm{E}+01$ & $23 \%$ \\
\hline${ }^{237} \mathrm{~Np}(\mathrm{IM})$ & $1.56 \mathrm{E}+00$ & $3.71 \mathrm{E}+00$ & $3.03 \mathrm{E}+00$ & $<2.31 \mathrm{E}+00$ & $2.76 \mathrm{E}+00$ & $40 \%$ \\
\hline${ }^{240} \mathrm{Pu}(\mathrm{IM})$ & $2.81 \mathrm{E}+00$ & $5.44 \mathrm{E}+00$ & $4.61 \mathrm{E}+00$ & $3.55 \mathrm{E}+00$ & $4.11 \mathrm{E}+00$ & $28 \%$ \\
\hline$<9.62 \mathrm{E}-01$ & $<9.88 \mathrm{E}-01$ & $<2.06 \mathrm{E}+00$ & $<1.85 \mathrm{E}+00$ & $<1.46 \mathrm{E}+00$ & - \\
\hline
\end{tabular}

Values in italics were not used in calculating the average. Divide $\mathrm{mg} / \mathrm{kg}$ values by $1 \mathrm{E}+04$ to convert to wt $\%$ basis. 
SRNL-STI-2012-00309

Revision 0

Table 3-4. Composition of the Water Leached Solids from Tank 16H Annulus Sample HTF-16-S-2 (continued)

\begin{tabular}{|c|c|c|c|c|c|c|}
\hline Analyte (Method) & $\begin{array}{c}\text { Aqua Regia } \\
\text { Replicate } 1 \\
\text { (dpm/g) } \\
\end{array}$ & $\begin{array}{c}\text { Aqua Regia } \\
\text { Replicate } 2 \\
\text { (dpm/g) }\end{array}$ & $\begin{array}{c}\text { Per Fusion } \\
\text { Replicate } 1 \\
\text { (dpm/g) }\end{array}$ & $\begin{array}{c}\text { Per Fusion } \\
\text { Replicate } 2 \\
\text { (dpm/g) }\end{array}$ & $\begin{array}{l}\text { Average } \\
\text { (dpm/g) }\end{array}$ & $\%$ RSD \\
\hline${ }^{90} \mathrm{Sr}$ (BS) & $1.45 \mathrm{E}+09$ & $3.23 \mathrm{E}+09$ & $2.53 \mathrm{E}+09$ & $1.79 \mathrm{E}+09$ & $2.25 \mathrm{E}+09$ & $35 \%$ \\
\hline${ }^{137} \mathrm{Cs}$ (GS) & $2.75 \mathrm{E}+09$ & $2.99 \mathrm{E}+09$ & $2.76 \mathrm{E}+09$ & $2.39 \mathrm{E}+09$ & $2.72 \mathrm{E}+09$ & $9.1 \%$ \\
\hline${ }^{238} \mathrm{Pu}(\mathrm{SA})$ & $2.69 \mathrm{E}+06$ & $6.66 \mathrm{E}+06$ & $4.71 \mathrm{E}+06$ & $3.70 \mathrm{E}+06$ & $4.44 \mathrm{E}+06$ & $38 \%$ \\
\hline${ }^{239 / 240} \mathrm{Pu}(\mathrm{SA})$ & $4.38 \mathrm{E}+05$ & $1.16 \mathrm{E}+06$ & $8.11 \mathrm{E}+05$ & $6.69 \mathrm{E}+05$ & $7.70 \mathrm{E}+05$ & $39 \%$ \\
\hline${ }^{99} \mathrm{Tc}$ (IM) & $4.97 \mathrm{E}+05$ & $7.08 \mathrm{E}+05$ & $7.58 \mathrm{E}+05$ & $4.86 \mathrm{E}+05$ & $6.13 \mathrm{E}+05$ & $23 \%$ \\
\hline${ }^{232} \mathrm{Th}$ (IM) & $3.86 \mathrm{E}-01$ & $7.93 \mathrm{E}-01$ & 7.13E-01 & 5.91E-01 & $6.21 \mathrm{E}-01$ & $29 \%$ \\
\hline${ }^{233} \mathrm{U}$ (IM) & $<2.06 \mathrm{E}+04$ & $<2.12 \mathrm{E}+04$ & $<2.20 \mathrm{E}+04$ & $<1.98 \mathrm{E}+04$ & $<2.09 \mathrm{E}+04$ & - \\
\hline${ }^{234} \mathrm{U}$ (IM) & $<1.34 \mathrm{E}+04$ & $<1.37 \mathrm{E}+04$ & $<1.43 \mathrm{E}+04$ & $<1.28 \mathrm{E}+04$ & $<1.36 \mathrm{E}+04$ & - \\
\hline${ }^{235} \mathrm{U}$ (IM) & $2.08 \mathrm{E}+01$ & $5.03 \mathrm{E}+01$ & $4.20 \mathrm{E}+01$ & $3.17 \mathrm{E}+01$ & $3.62 \mathrm{E}+01$ & $35 \%$ \\
\hline${ }^{236} \mathrm{U}$ (IM) & $<1.38 \mathrm{E}+02$ & $<1.42 \mathrm{E}+02$ & $<2.95 \mathrm{E}+02$ & $<2.65 \mathrm{E}+02$ & $<2.10 \mathrm{E}+02$ & \\
\hline${ }^{237} \mathrm{~Np}$ (IM) & $2.43 \mathrm{E}+03$ & $5.80 \mathrm{E}+03$ & $4.74 \mathrm{E}+03$ & $<3.61 \mathrm{E}+03$ & $4.33 \mathrm{E}+03$ & $40 \%$ \\
\hline${ }^{238} \mathrm{U}(\mathrm{IM})$ & $7.09 \mathrm{E}+01$ & $1.92 \mathrm{E}+02$ & $1.67 \mathrm{E}+02$ & $1.14 \mathrm{E}+02$ & $1.36 \mathrm{E}+02$ & $40 \%$ \\
\hline${ }^{239} \mathrm{Pu}$ (IM) & $3.83 \mathrm{E}+05$ & $7.41 \mathrm{E}+05$ & $6.28 \mathrm{E}+05$ & $4.84 \mathrm{E}+05$ & $5.59 \mathrm{E}+05$ & $28 \%$ \\
\hline${ }^{240} \mathrm{Pu}$ (IM) & $<4.82 \mathrm{E}+05$ & $<4.95 \mathrm{E}+05$ & $<1.03 \mathrm{E}+06$ & $<9.24 \mathrm{E}+05$ & $<7.33 \mathrm{E}+05$ & - \\
\hline
\end{tabular}

Values in italics were not used in calculating the average. Divide $\mathrm{mg} / \mathrm{kg}$ values by $1 \mathrm{E}+04$ to convert to wt $\%$ basis. 
SRNL-STI-2012-00309

Revision 0

Table 3-5. Composition of the Water Leached Solids from Tank 16H Annulus Sample HTF-16-W-1

\begin{tabular}{|c|c|c|c|c|c|c|}
\hline Analyte (Method) & $\begin{array}{c}\text { Aqua Regia } \\
\text { Replicate } 1 \\
\text { (mg/kg) }\end{array}$ & $\begin{array}{c}\text { Aqua Regia } \\
\text { Replicate } 2 \\
\text { (mg/kg) }\end{array}$ & $\begin{array}{c}\text { Per Fusion } \\
\text { Replicate } 1 \\
\text { (mg/kg) }\end{array}$ & $\begin{array}{c}\text { Per Fusion } \\
\text { Replicate } 2 \\
\text { (mg/kg) }\end{array}$ & $\begin{array}{c}\text { Average } \\
(\mathbf{m g} / \mathbf{k g})\end{array}$ & $\%$ RSD \\
\hline $\mathrm{Ag}$ (IE) & $<1.12 \mathrm{E}+02$ & $<9.90 \mathrm{E}+01$ & $<9.47 E+02$ & $<9.57 E+02$ & $<1.06 \mathrm{E}+02$ & - \\
\hline $\mathrm{Al}$ (IE) & $1.26 \mathrm{E}+05$ & $1.04 \mathrm{E}+05$ & $1.39 \mathrm{E}+05$ & $1.24 \mathrm{E}+05$ & $1.23 \mathrm{E}+05$ & $12 \%$ \\
\hline B (IE) & $<1.57 \mathrm{E}+01$ & $<1.39 \mathrm{E}+01$ & $<1.33 E+02$ & $<1.34 E+02$ & $<1.48 \mathrm{E}+01$ & - \\
\hline $\mathrm{Ba}$ (IE) & $1.54 \mathrm{E}+02$ & $1.46 \mathrm{E}+02$ & $1.29 \mathrm{E}+02$ & $1.25 \mathrm{E}+02$ & $1.39 \mathrm{E}+02$ & $10 \%$ \\
\hline $\mathrm{Be}$ (IE) & $<4.65 \mathrm{E}-01$ & $<4.12 \mathrm{E}-01$ & $<5.26 E+00$ & $<5.32 E+00$ & $<4.39 \mathrm{E}-01$ & - \\
\hline $\mathrm{Ca}$ (IE) & $1.41 \mathrm{E}+03$ & $2.23 \mathrm{E}+04$ & $3.14 E+03$ & $3.74 E+03$ & $1.19 \mathrm{E}+04$ & $125 \%$ \\
\hline $\mathrm{Cd}$ (IE) & $5.58 \mathrm{E}+00$ & $4.74 \mathrm{E}+00$ & $<2.04 E+01$ & $<2.06 E+01$ & $5.16 \mathrm{E}+00$ & $12 \%$ \\
\hline $\mathrm{Ce}$ (IE) & $2.23 \mathrm{E}+02$ & $2.25 \mathrm{E}+02$ & $<4.65 E+02$ & $<4.70 E+02$ & $2.24 \mathrm{E}+02$ & $0.6 \%$ \\
\hline Co (IE) & $<7.52 \mathrm{E}+00$ & $<6.67 \mathrm{E}+00$ & $<6.38 E+01$ & $<6.45 E+01$ & $<7.10 \mathrm{E}+00$ & - \\
\hline Cr (IE) & $1.38 \mathrm{E}+02$ & $2.04 \mathrm{E}+02$ & $9.77 \mathrm{E}+01$ & $1.07 \mathrm{E}+02$ & $1.37 \mathrm{E}+02$ & $35 \%$ \\
\hline $\mathrm{Cu}$ (IE) & $1.54 \mathrm{E}+02$ & $1.29 \mathrm{E}+02$ & $1.11 \mathrm{E}+02$ & $1.20 \mathrm{E}+02$ & $1.29 \mathrm{E}+02$ & $14 \%$ \\
\hline $\mathrm{Fe}(\mathrm{IE})$ & $3.48 \mathrm{E}+04$ & $2.76 \mathrm{E}+04$ & $1.55 \mathrm{E}+04$ & $2.04 \mathrm{E}+04$ & $2.46 \mathrm{E}+04$ & $34 \%$ \\
\hline Gd (IE) & $<1.67 \mathrm{E}+01$ & $<1.48 \mathrm{E}+01$ & $<1.41 E+02$ & $<1.43 E+02$ & $<1.58 \mathrm{E}+01$ & - \\
\hline $\mathrm{K}$ (IE) & $8.58 \mathrm{E}+02$ & $7.23 \mathrm{E}+02$ & $4.16 E+03$ & $3.77 E+03$ & $7.91 \mathrm{E}+02$ & $12 \%$ \\
\hline La (IE) & $8.97 \mathrm{E}+01$ & $8.82 \mathrm{E}+01$ & $7.17 \mathrm{E}+01$ & $7.51 \mathrm{E}+01$ & $8.12 \mathrm{E}+01$ & $11 \%$ \\
\hline Li (IE) & $<1.13 \mathrm{E}+01$ & $<1.00 \mathrm{E}+01$ & $<9.61 E+01$ & $<9.70 E+01$ & $<1.07 \mathrm{E}+01$ & - \\
\hline $\mathrm{Mg}$ (IE) & $2.80 \mathrm{E}+02$ & $6.95 \mathrm{E}+03$ & $2.38 \mathrm{E}+02$ & $2.68 \mathrm{E}+02$ & $1.93 \mathrm{E}+03$ & $173 \%$ \\
\hline Mn (IE) & $2.88 \mathrm{E}+02$ & $3.08 \mathrm{E}+02$ & $2.00 \mathrm{E}+02$ & $2.11 \mathrm{E}+02$ & $2.52 \mathrm{E}+02$ & $22 \%$ \\
\hline Mo (IE) & $<4.78 \mathrm{E}+01$ & $<4.24 \mathrm{E}+01$ & $<1.76 E+02$ & $<1.78 E+02$ & $<4.51 \mathrm{E}+01$ & - \\
\hline $\mathrm{Na}$ (IE) & $1.32 \mathrm{E}+05$ & $1.10 \mathrm{E}+05$ & - & - & $1.21 \mathrm{E}+05$ & $13 \%$ \\
\hline $\mathrm{Ni}$ (IE) & $1.49 \mathrm{E}+02$ & $1.82 \mathrm{E}+02$ & $<2.99 E+02$ & $<3.02 E+02$ & $1.66 \mathrm{E}+02$ & $14 \%$ \\
\hline $\mathrm{P}$ (IE) & $<6.71 \mathrm{E}+01$ & $<5.95 \mathrm{E}+01$ & $<5.70 E+02$ & $<5.75 E+02$ & $<6.33 \mathrm{E}+01$ & - \\
\hline $\mathrm{Pb}$ (IE) & $7.37 \mathrm{E}+02$ & $5.99 \mathrm{E}+02$ & $6.55 \mathrm{E}+02$ & $5.48 \mathrm{E}+02$ & $6.35 \mathrm{E}+02$ & $13 \%$ \\
\hline S (IE) & $<5.81 \mathrm{E}+02$ & $<5.15 \mathrm{E}+02$ & $<4.93 E+03$ & $<4.98 E+03$ & $<5.48 \mathrm{E}+02$ & - \\
\hline $\mathrm{Sb}$ (IE) & $<9.90 \mathrm{E}+01$ & $<8.78 \mathrm{E}+01$ & $<7.01 E+02$ & $<7.08 E+02$ & $<9.34 \mathrm{E}+01$ & - \\
\hline Si (IE) & - & - & $1.74 \mathrm{E}+05$ & $1.75 \mathrm{E}+05$ & $1.75 \mathrm{E}+05$ & $0.4 \%$ \\
\hline Sn (IE) & $<4.35 \mathrm{E}+01$ & $<3.86 \mathrm{E}+01$ & $<3.69 E+02$ & $<3.73 E+02$ & $<4.11 \mathrm{E}+01$ & - \\
\hline
\end{tabular}

Values in italics were not used in calculating the average. Divide $\mathrm{mg} / \mathrm{kg}$ values by $1 \mathrm{E}+04$ to convert to wt $\%$ basis. 
SRNL-STI-2012-00309

Revision 0

Table 3-5. Composition of the Water Leached Solids from Tank 16H Annulus Sample HTF-16-W-1 (continued)

\begin{tabular}{|c|c|c|c|c|c|c|}
\hline Analyte (Method) & $\begin{array}{c}\text { Aqua Regia } \\
\text { Replicate } 1 \\
\text { (mg/kg) }\end{array}$ & $\begin{array}{c}\text { Aqua Regia } \\
\text { Replicate } 2 \\
\text { (mg/kg) }\end{array}$ & $\begin{array}{c}\text { Per Fusion } \\
\text { Replicate } 1 \\
\text { (mg/kg) }\end{array}$ & $\begin{array}{c}\text { Per Fusion } \\
\text { Replicate } 2 \\
\text { (mg/kg) }\end{array}$ & $\begin{array}{c}\text { Average } \\
\text { (mg/kg) }\end{array}$ & $\%$ RSD \\
\hline $\mathrm{Sr}$ (IE) & $5.36 \mathrm{E}+01$ & $5.40 \mathrm{E}+01$ & $7.14 E+01$ & $7.08 E+01$ & $5.38 \mathrm{E}+01$ & $0.5 \%$ \\
\hline Th (IE) & $<4.15 \mathrm{E}+01$ & $<3.68 \mathrm{E}+01$ & $<3.61 E+02$ & $<3.64 E+02$ & $<3.92 \mathrm{E}+01$ & - \\
\hline $\mathrm{Ti}$ (IE) & $4.77 E+01$ & $8.12 E+01$ & $7.43 \mathrm{E}+01$ & $7.22 \mathrm{E}+02$ & $3.98 \mathrm{E}+02$ & $115 \%$ \\
\hline $\mathrm{U}$ (IE) & $3.39 \mathrm{E}+02$ & $1.08 \mathrm{E}+03$ & $<2.15 E+03$ & $<2.17 E+03$ & $7.10 \mathrm{E}+02$ & $74 \%$ \\
\hline V (IE) & $<3.64 \mathrm{E}+00$ & $<3.23 \mathrm{E}+00$ & $<3.09 E+01$ & $<3.12 E+01$ & $<3.44 \mathrm{E}+00$ & - \\
\hline Zn (IE) & $4.43 \mathrm{E}+02$ & $2.92 \mathrm{E}+02$ & $3.30 \mathrm{E}+02$ & $4.33 \mathrm{E}+02$ & $3.75 \mathrm{E}+02$ & $20 \%$ \\
\hline $\mathrm{Zr}$ (IE) & $2.65 \mathrm{E}+02$ & $2.58 \mathrm{E}+02$ & - & - & $2.62 \mathrm{E}+02$ & $1.9 \%$ \\
\hline $\mathrm{Hg}(\mathrm{CV})$ & $2.69 \mathrm{E}+03$ & $2.37 \mathrm{E}+03$ & - & - & $2.53 \mathrm{E}+03$ & $9 \%$ \\
\hline${ }^{232} \mathrm{Th}(\mathrm{IM})$ & $1.55 \mathrm{E}+00$ & $1.61 \mathrm{E}+00$ & $1.99 \mathrm{E}+00$ & $1.44 \mathrm{E}+00$ & $1.65 \mathrm{E}+00$ & $15 \%$ \\
\hline${ }^{233} \mathrm{U}(\mathrm{IM})$ & $<9.69 \mathrm{E}-01$ & $<8.59 \mathrm{E}-01$ & $<8.22 \mathrm{E}-01$ & $<8.31 \mathrm{E}-01$ & $<8.70 \mathrm{E}-01$ & - \\
\hline${ }^{234} \mathrm{U}(\mathrm{IM})$ & $<9.69 \mathrm{E}-01$ & $<8.59 \mathrm{E}-01$ & $<8.22 \mathrm{E}-01$ & $<8.31 \mathrm{E}-01$ & $<8.70 \mathrm{E}-01$ & - \\
\hline${ }^{235} \mathrm{U}(\mathrm{IM})$ & $1.07 \mathrm{E}+01$ & $9.85 \mathrm{E}+00$ & $1.21 \mathrm{E}+01$ & $1.05 \mathrm{E}+01$ & $1.08 \mathrm{E}+01$ & $8.8 \%$ \\
\hline${ }^{236} \mathrm{U}(\mathrm{IM})$ & $<9.69 \mathrm{E}-01$ & $<1.43 \mathrm{E}+00$ & $<1.64 \mathrm{E}+00$ & $<1.66 \mathrm{E}+00$ & $<1.43 \mathrm{E}+00$ & - \\
\hline${ }^{238} \mathrm{U}(\mathrm{IM})$ & $2.67 \mathrm{E}+02$ & $2.69 \mathrm{E}+02$ & $3.04 \mathrm{E}+02$ & $2.72 \mathrm{E}+02$ & $2.78 \mathrm{E}+02$ & $6.3 \%$ \\
\hline U-total (IM) & $2.77 \mathrm{E}+02$ & $2.78 \mathrm{E}+02$ & $3.16 \mathrm{E}+02$ & $2.83 \mathrm{E}+02$ & $2.89 \mathrm{E}+02$ & $6.4 \%$ \\
\hline${ }^{90} \mathrm{Sr}(\mathrm{BS})$ & $6.47 \mathrm{E}+00$ & $8.31 \mathrm{E}+00$ & $6.70 \mathrm{E}+00$ & $8.12 \mathrm{E}+00$ & $7.40 \mathrm{E}+00$ & $13 \%$ \\
\hline${ }^{137} \mathrm{Cs}$ (GS) & $2.13 \mathrm{E}+01$ & $1.79 \mathrm{E}+01$ & $2.37 \mathrm{E}+01$ & $2.18 \mathrm{E}+01$ & $2.11 \mathrm{E}+01$ & $11 \%$ \\
\hline${ }^{238} \mathrm{Pu}(\mathrm{SA})$ & $1.71 \mathrm{E}-01$ & $1.81 \mathrm{E}-01$ & $1.63 \mathrm{E}-01$ & $1.54 \mathrm{E}-01$ & $1.67 \mathrm{E}-01$ & $6.9 \%$ \\
\hline${ }^{99} \mathrm{Tc}(\mathrm{IM})$ & $2.46 \mathrm{E}+01$ & $2.18 \mathrm{E}+01$ & $2.46 \mathrm{E}+01$ & $2.24 \mathrm{E}+01$ & $2.33 \mathrm{E}+01$ & $6.3 \%$ \\
\hline${ }^{237} \mathrm{~Np}$ (IM) & $2.82 \mathrm{E}+00$ & $2.95 \mathrm{E}+00$ & $3.75 \mathrm{E}+00$ & $3.58 \mathrm{E}+00$ & $3.27 \mathrm{E}+00$ & $14 \%$ \\
\hline${ }^{239} \mathrm{Pu}$ (IM) & $6.39 \mathrm{E}+00$ & $6.66 \mathrm{E}+00$ & $6.88 \mathrm{E}+00$ & $6.56 \mathrm{E}+00$ & $6.62 \mathrm{E}+00$ & $3.1 \%$ \\
\hline${ }^{240} \mathrm{Pu}$ (IM) & $<9.69 \mathrm{E}-01$ & $<8.59 \mathrm{E}-01$ & $<1.64 \mathrm{E}+00$ & $<1.66 \mathrm{E}+00$ & $<1.28 \mathrm{E}+00$ & - \\
\hline
\end{tabular}

Values in italics were not used in calculating the average. Divide $\mathrm{mg} / \mathrm{kg}$ values by $1 \mathrm{E}+04$ to convert to wt $\%$ basis. 
SRNL-STI-2012-00309

Revision 0

Table 3-5. Composition of the Water Leached Solids from Tank 16H Annulus Sample HTF-16-W-1 (continued)

\begin{tabular}{|c|c|c|c|c|c|c|}
\hline Analyte (Method) & $\begin{array}{c}\text { Aqua Regia } \\
\text { Replicate } 1 \\
\text { (dpm/g) }\end{array}$ & $\begin{array}{c}\text { Aqua Regia } \\
\text { Replicate } 2 \\
\text { (dpm/g) }\end{array}$ & $\begin{array}{c}\text { Per Fusion } \\
\text { Replicate } 1 \\
\text { (dpm/g) }\end{array}$ & $\begin{array}{c}\text { Per Fusion } \\
\text { Replicate } 2 \\
\text { (dpm/g) }\end{array}$ & $\begin{array}{l}\text { Average } \\
(\mathrm{dpm} / \mathrm{g})\end{array}$ & $\%$ RSD \\
\hline${ }^{90} \mathrm{Sr}(\mathrm{BS})$ & $1.97 \mathrm{E}+09$ & $2.53 \mathrm{E}+09$ & $2.04 \mathrm{E}+09$ & $2.47 \mathrm{E}+09$ & $2.25 \mathrm{E}+09$ & $13 \%$ \\
\hline${ }^{137} \mathrm{Cs}$ (GS) & $4.10 \mathrm{E}+09$ & $3.44 \mathrm{E}+09$ & $4.56 \mathrm{E}+09$ & $4.19 \mathrm{E}+09$ & $4.07 \mathrm{E}+09$ & $11 \%$ \\
\hline${ }^{238} \mathrm{Pu}(\mathrm{SA})$ & $6.50 \mathrm{E}+06$ & $6.88 \mathrm{E}+06$ & $6.21 \mathrm{E}+06$ & $5.85 \mathrm{E}+06$ & $6.36 \mathrm{E}+06$ & $6.9 \%$ \\
\hline${ }^{239 / 240} \mathrm{Pu}$ (SA) & $1.23 \mathrm{E}+06$ & $1.28 \mathrm{E}+06$ & $1.15 \mathrm{E}+06$ & $1.03 \mathrm{E}+06$ & $1.17 \mathrm{E}+06$ & $9.3 \%$ \\
\hline${ }^{99}$ Tc (IM) & $9.25 \mathrm{E}+05$ & $8.22 \mathrm{E}+05$ & $9.28 \mathrm{E}+05$ & $8.42 \mathrm{E}+05$ & $8.79 \mathrm{E}+05$ & $6.3 \%$ \\
\hline${ }^{232} \mathrm{Th}$ (IM) & $3.78 \mathrm{E}-01$ & $3.94 \mathrm{E}-01$ & $4.87 \mathrm{E}-01$ & $3.50 \mathrm{E}-01$ & $4.02 \mathrm{E}-01$ & $15 \%$ \\
\hline${ }^{233} \mathrm{U}(\mathrm{IM})$ & $<2.08 \mathrm{E}+04$ & $<1.84 \mathrm{E}+04$ & $<1.76 \mathrm{E}+04$ & $<1.78 \mathrm{E}+04$ & $<1.86 \mathrm{E}+04$ & - \\
\hline${ }^{234} \mathrm{U}(\mathrm{IM})$ & $<1.35 \mathrm{E}+04$ & $<1.19 \mathrm{E}+04$ & $<1.14 \mathrm{E}+04$ & $<1.15 \mathrm{E}+04$ & $<1.21 \mathrm{E}+04$ & - \\
\hline${ }^{235} \mathrm{U}$ (IM) & $5.14 \mathrm{E}+01$ & $4.72 \mathrm{E}+01$ & $5.81 \mathrm{E}+01$ & $5.04 \mathrm{E}+01$ & $5.18 \mathrm{E}+01$ & $8.8 \%$ \\
\hline${ }^{236} \mathrm{U}$ (IM) & $<1.39 \mathrm{E}+02$ & $<2.05 \mathrm{E}+02$ & $<2.36 \mathrm{E}+02$ & $<2.38 \mathrm{E}+02$ & $<2.05 \mathrm{E}+02$ & - \\
\hline${ }^{237} \mathrm{~Np}$ (IM) & $4.41 \mathrm{E}+03$ & $4.62 \mathrm{E}+03$ & $5.87 \mathrm{E}+03$ & $5.60 \mathrm{E}+03$ & $5.12 \mathrm{E}+03$ & $14 \%$ \\
\hline${ }^{238} \mathrm{U}(\mathrm{IM})$ & $1.99 \mathrm{E}+02$ & $2.00 \mathrm{E}+02$ & $2.27 \mathrm{E}+02$ & $2.03 \mathrm{E}+02$ & $2.07 \mathrm{E}+02$ & $6.3 \%$ \\
\hline${ }^{239} \mathrm{Pu}$ (IM) & $8.70 \mathrm{E}+05$ & $9.06 \mathrm{E}+05$ & $9.37 \mathrm{E}+05$ & $8.93 \mathrm{E}+05$ & $9.02 \mathrm{E}+05$ & $3.1 \%$ \\
\hline${ }^{240} \mathrm{Pu}$ (IM) & $<4.85 \mathrm{E}+05$ & $<4.30 \mathrm{E}+05$ & $<8.24 \mathrm{E}+05$ & $<8.32 \mathrm{E}+05$ & $<6.43 \mathrm{E}+05$ & - \\
\hline
\end{tabular}

Values in italics were not used in calculating the average. Divide $\mathrm{mg} / \mathrm{kg}$ values by $1 \mathrm{E}+04$ to convert to wt $\%$ basis. 
Table 3-6. Composition of the Oxalic Acid Leachates from Tank 16H Annulus Sample HTF-16-E-2 from Three Leaching Cycles

\begin{tabular}{|c|c|c|c|c|c|}
\hline Analyte (Method) & $\begin{array}{c}\text { Decant } 1 \\
(\mathrm{mg} / \mathrm{L})\end{array}$ & $\begin{array}{c}\text { Decant } 2 \\
(\mathrm{mg} / \mathrm{L}) \\
\end{array}$ & $\begin{array}{c}\text { Decant } 3 \\
(\mathrm{mg} / \mathrm{L}) \\
\end{array}$ & $\frac{\text { Decant } 2}{\text { Decant } 1}$ & $\frac{\text { Decant 3 }}{\text { Decant } 2}$ \\
\hline $\mathrm{F}^{-}(\mathrm{IC})$ & $<1.00 \mathrm{E}+02$ & $<1.00 \mathrm{E}+02$ & $<1.00 \mathrm{E}+02$ & - & - \\
\hline $\mathrm{CH}_{2} \mathrm{O}^{-}$(IC) & $<1.00 \mathrm{E}+02$ & $<1.00 \mathrm{E}+02$ & $<1.00 \mathrm{E}+02$ & - & - \\
\hline $\mathrm{Cl}^{-}$(IC) & $4.17 \mathrm{E}+02$ & $1.75 \mathrm{E}+02$ & $<1.00 \mathrm{E}+02$ & 0.42 & - \\
\hline $\mathrm{NO}_{2}^{-}$(IC) & $5.60 \mathrm{E}+03$ & $<1.00 \mathrm{E}+02$ & $<1.00 \mathrm{E}+02$ & - & - \\
\hline $\mathrm{Br}^{-}$(IC) & $<1.00 \mathrm{E}+03$ & $<1.00 \mathrm{E}+03$ & $<1.00 \mathrm{E}+03$ & - & - \\
\hline $\mathrm{NO}_{3}^{-}(\mathrm{IC})$ & $1.24 \mathrm{E}+04$ & $9.68 \mathrm{E}+03$ & $3.20 \mathrm{E}+03$ & 0.78 & 0.33 \\
\hline $\mathrm{PO}_{4}^{3-}(\mathrm{IC})$ & $<1.00 \mathrm{E}+02$ & $<1.00 \mathrm{E}+02$ & $<1.00 \mathrm{E}+02$ & - & - \\
\hline $\mathrm{SO}_{4}^{2-}(\mathrm{IC})$ & $<1.20 \mathrm{E}+03$ & $<1.20 \mathrm{E}+03$ & $<1.20 \mathrm{E}+03$ & - & - \\
\hline $\mathrm{C}_{2} \mathrm{O}_{4}{ }^{2-}$ (IC) & $3.89 \mathrm{E}+04$ & $3.83 \mathrm{E}+04$ & $3.67 \mathrm{E}+04$ & 0.98 & 0.96 \\
\hline $\operatorname{Ag}(\mathrm{IE})$ & $<1.46 \mathrm{E}+00$ & $<1.46 \mathrm{E}+00$ & $<1.46 \mathrm{E}+00$ & - & - \\
\hline $\mathrm{Al}$ (IE) & $4.27 \mathrm{E}+03$ & $4.39 \mathrm{E}+03$ & $2.16 \mathrm{E}+03$ & 1.03 & 0.49 \\
\hline B (IE) & $<2.02 \mathrm{E}+00$ & $<2.02 \mathrm{E}+00$ & $<2.02 \mathrm{E}+00$ & - & - \\
\hline $\mathrm{Ba}$ (IE) & $1.50 \mathrm{E}+00$ & $2.44 \mathrm{E}+00$ & $2.35 \mathrm{E}+00$ & 1.63 & 0.96 \\
\hline $\mathrm{Be}$ (IE) & $<6.00 \mathrm{E}-02$ & $<6.00 \mathrm{E}-02$ & $<6.00 \mathrm{E}-02$ & - & - \\
\hline $\mathrm{Ca}$ (IE) & $3.52 \mathrm{E}+01$ & $6.08 \mathrm{E}+01$ & $4.65 \mathrm{E}+01$ & 1.73 & 0.76 \\
\hline $\mathrm{Cd}(\mathrm{IE})$ & $<6.40 \mathrm{E}-01$ & $<6.40 \mathrm{E}-01$ & $<6.40 \mathrm{E}-01$ & - & - \\
\hline $\mathrm{Ce}$ (IE) & $<7.07 \mathrm{E}+00$ & $<7.07 \mathrm{E}+00$ & $<7.07 \mathrm{E}+00$ & - & - \\
\hline Co (IE) & $<9.70 \mathrm{E}-01$ & $<9.70 \mathrm{E}-01$ & $<9.70 \mathrm{E}-01$ & - & - \\
\hline Cr (IE) & $1.48 \mathrm{E}+01$ & $2.73 \mathrm{E}+01$ & $1.97 \mathrm{E}+01$ & 1.84 & 0.72 \\
\hline $\mathrm{Cu}$ (IE) & $8.59 \mathrm{E}+00$ & $8.57 \mathrm{E}+00$ & $4.66 \mathrm{E}+00$ & 1.00 & 0.54 \\
\hline $\mathrm{Fe}(\mathrm{IE})$ & $1.38 \mathrm{E}+02$ & $1.87 \mathrm{E}+02$ & $3.97 \mathrm{E}+02$ & 1.36 & 2.12 \\
\hline Gd (IE) & $<7.80 \mathrm{E}-01$ & $<7.80 \mathrm{E}-01$ & $<7.80 \mathrm{E}-01$ & - & - \\
\hline K (IE) & $1.64 \mathrm{E}+02$ & $1.40 \mathrm{E}+02$ & $7.41 \mathrm{E}+01$ & 0.85 & 0.53 \\
\hline La (IE) & $<6.70 \mathrm{E}-01$ & $<6.70 \mathrm{E}-01$ & $<6.70 \mathrm{E}-01$ & - & - \\
\hline Li (IE) & $1.77 \mathrm{E}+00$ & $<1.46 \mathrm{E}+00$ & $<1.46 \mathrm{E}+00$ & - & - \\
\hline $\mathrm{Mg}$ (IE) & $3.41 \mathrm{E}+01$ & $3.94 \mathrm{E}+01$ & $3.02 \mathrm{E}+01$ & 1.16 & 0.77 \\
\hline Mn (IE) & $5.50 \mathrm{E}+00$ & $6.64 \mathrm{E}+00$ & $5.70 \mathrm{E}+00$ & 1.21 & 0.86 \\
\hline Mo (IE) & $<6.17 \mathrm{E}+00$ & $<6.17 \mathrm{E}+00$ & $<6.17 \mathrm{E}+00$ & - & - \\
\hline
\end{tabular}


Table 3-6. Composition of the Oxalic Acid Leachates from Tank 16H Annulus Sample HTF-16-E-2 from Three Leaching Cycles (continued)

\begin{tabular}{|c|c|c|c|c|c|}
\hline Analyte (Method) & $\begin{array}{c}\text { Decant } 1 \\
(\mathrm{mg} / \mathrm{L})\end{array}$ & $\begin{array}{c}\text { Decant } 2 \\
(\mathrm{mg} / \mathrm{L}) \\
\end{array}$ & $\begin{array}{c}\text { Decant } 3 \\
(\mathrm{mg} / \mathrm{L}) \\
\end{array}$ & $\frac{\text { Decant } 2}{\text { Decant } 1}$ & $\begin{array}{l}\text { Decant } 3 \\
\text { Decant } 2 \\
\end{array}$ \\
\hline $\mathrm{Na}$ (IE) & $1.71 \mathrm{E}+04$ & $1.19 \mathrm{E}+04$ & $5.69 \mathrm{E}+03$ & 0.70 & 0.48 \\
\hline Ni (IE) & $<4.55 \mathrm{E}+00$ & $<4.55 \mathrm{E}+00$ & $<4.55 \mathrm{E}+00$ & - & - \\
\hline P (IE) & $<8.66 \mathrm{E}+00$ & $<8.66 \mathrm{E}+00$ & $<8.66 \mathrm{E}+00$ & - & - \\
\hline $\mathrm{Pb}$ (IE) & $<7.16 \mathrm{E}+00$ & $<7.16 \mathrm{E}+00$ & $<7.16 \mathrm{E}+00$ & - & - \\
\hline S (IE) & $3.33 \mathrm{E}+02$ & $2.04 \mathrm{E}+02$ & $1.18 \mathrm{E}+02$ & 0.61 & 0.58 \\
\hline $\mathrm{Sb}$ (IE) & $<1.07 \mathrm{E}+01$ & $<1.07 \mathrm{E}+01$ & $<1.07 \mathrm{E}+01$ & - & - \\
\hline Si (IE) & $9.39 \mathrm{E}+01$ & $5.64 \mathrm{E}+02$ & $2.72 \mathrm{E}+02$ & 6.01 & 0.48 \\
\hline Sn (IE) & $<5.61 \mathrm{E}+00$ & $<5.61 \mathrm{E}+00$ & $<5.61 \mathrm{E}+00$ & - & - \\
\hline Sr (IE) & $7.50 \mathrm{E}-01$ & $6.00 \mathrm{E}-01$ & $6.20 \mathrm{E}-01$ & 0.80 & 1.03 \\
\hline Th (IE) & $<2.68 \mathrm{E}+00$ & $<2.68 \mathrm{E}+00$ & $<2.68 \mathrm{E}+00$ & - & - \\
\hline Ti (IE) & $<1.30 \mathrm{E}-01$ & $1.60 \mathrm{E}-01$ & $8.10 \mathrm{E}-01$ & - & - \\
\hline U (IE) & $<3.27 \mathrm{E}+01$ & $<3.27 \mathrm{E}+01$ & $<3.27 \mathrm{E}+01$ & - & - \\
\hline V (IE) & $<4.70 \mathrm{E}-01$ & $<4.70 \mathrm{E}-01$ & $<4.70 \mathrm{E}-01$ & - & - \\
\hline Zn (IE) & $4.58 \mathrm{E}+01$ & $4.58 \mathrm{E}+01$ & $2.39 \mathrm{E}+01$ & 1.00 & 0.52 \\
\hline Zr (IE) & 8.80E-01 & $1.36 \mathrm{E}+00$ & $2.27 \mathrm{E}+00$ & 1.55 & 1.67 \\
\hline $\mathrm{Hg}(\mathrm{CV})$ & $3.37 \mathrm{E}+01$ & $4.11 \mathrm{E}+01$ & $2.36 \mathrm{E}+01$ & 1.22 & 0.57 \\
\hline${ }^{232} \mathrm{Th}$ (IM) & $7.50 \mathrm{E}-02$ & $7.50 \mathrm{E}-02$ & $3.00 \mathrm{E}-02$ & 1.00 & 0.40 \\
\hline${ }^{233} \mathrm{U}(\mathrm{IM})$ & $<5.00 \mathrm{E}-02$ & $<5.00 \mathrm{E}-02$ & $<2.00 \mathrm{E}-02$ & - & - \\
\hline${ }^{234} \mathrm{U}(\mathrm{IM})$ & $<5.00 \mathrm{E}-02$ & $<5.00 \mathrm{E}-02$ & $<2.00 \mathrm{E}-02$ & - & - \\
\hline${ }^{235} \mathrm{U}(\mathrm{IM})$ & $1.13 \mathrm{E}+00$ & $8.58 \mathrm{E}-01$ & $3.35 \mathrm{E}-01$ & 0.76 & 0.39 \\
\hline${ }^{236} \mathrm{U}(\mathrm{IM})$ & $1.07 \mathrm{E}-01$ & 8.17E-02 & $3.65 \mathrm{E}-02$ & 0.76 & 0.45 \\
\hline${ }^{238} \mathrm{U}$ (IM) & $2.97 \mathrm{E}+01$ & $2.22 \mathrm{E}+01$ & $8.62 \mathrm{E}+00$ & 0.75 & 0.39 \\
\hline U-total (IM) & $3.09 \mathrm{E}+01$ & $2.31 \mathrm{E}+01$ & $9.00 \mathrm{E}+00$ & 0.75 & 0.39 \\
\hline${ }^{137} \mathrm{Cs}$ (GS) & $3.17 \mathrm{E}-01$ & 4.89E-01 & 4.73E-01 & 1.54 & 0.97 \\
\hline${ }^{99}$ Tc (IM) & $9.49 \mathrm{E}-01$ & $6.19 \mathrm{E}-01$ & $2.33 \mathrm{E}-01$ & 0.65 & 0.38 \\
\hline${ }^{237} \mathrm{~Np}$ (IM) & $1.51 \mathrm{E}-01$ & $1.48 \mathrm{E}-01$ & 4.12E-02 & 0.98 & 0.28 \\
\hline${ }^{239} \mathrm{Pu}$ (IM) & $7.18 \mathrm{E}-02$ & 7.91E-02 & $5.75 \mathrm{E}-02$ & 1.10 & 0.73 \\
\hline${ }^{240} \mathrm{Pu}$ (IM) & $<5.00 \mathrm{E}-02$ & $<5.00 \mathrm{E}-02$ & $<2.00 \mathrm{E}-02$ & - & - \\
\hline $\mathrm{pH}$ (pH units) & 5.06 & 4.38 & 1.29 & - & - \\
\hline
\end{tabular}


Table 3-6. Composition of the Oxalic Acid Leachates from Tank 16H Annulus Sample HTF-16-E-2 from Three Leaching Cycles (continued)

\begin{tabular}{|l|c|c|c|c|c||}
\hline Analyte (Method) & $\begin{array}{c}\text { Decant 1 } \\
(\mathbf{d p m} / \mathbf{m l})\end{array}$ & $\begin{array}{c}\text { Decant 2 } \\
(\mathbf{d p m} / \mathbf{m l})\end{array}$ & $\begin{array}{c}\text { Decant 3 } \\
(\mathbf{d p m} / \mathbf{m l})\end{array}$ & $\begin{array}{c}\text { Decant 2 } \\
\text { Decant 1 }\end{array}$ & $\begin{array}{c}\text { Decant 3 } \\
\text { Decant 2 }\end{array}$ \\
\hline${ }^{137} \mathrm{Cs}(\mathrm{GS})$ & $6.10 \mathrm{E}+07$ & $9.42 \mathrm{E}+07$ & $9.11 \mathrm{E}+07$ & 1.54 & 0.97 \\
\hline${ }^{99} \mathrm{Tc}(\mathrm{IM})$ & $3.58 \mathrm{E}+04$ & $2.33 \mathrm{E}+04$ & $8.77 \mathrm{E}+03$ & 0.65 & 0.38 \\
\hline${ }^{232} \mathrm{Th}(\mathrm{IM})$ & $1.83 \mathrm{E}-02$ & $1.83 \mathrm{E}-02$ & $7.33 \mathrm{E}-03$ & 1.00 & 0.40 \\
\hline${ }^{233} \mathrm{U}(\mathrm{IM})$ & $<1.07 \mathrm{E}+03$ & $<1.07 \mathrm{E}+03$ & $<4.28 \mathrm{E}+02$ & - & - \\
\hline${ }^{234} \mathrm{U}(\mathrm{IM})$ & $<6.95 \mathrm{E}+02$ & $<6.95 \mathrm{E}+02$ & $<2.78 \mathrm{E}+02$ & - & - \\
\hline${ }^{235} \mathrm{U}(\mathrm{IM})$ & $5.43 \mathrm{E}+00$ & $4.11 \mathrm{E}+00$ & $1.61 \mathrm{E}+00$ & 0.76 & 0.39 \\
\hline${ }^{237} \mathrm{~Np}(\mathrm{IM})$ & $1.53 \mathrm{E}+01$ & $1.17 \mathrm{E}+01$ & $5.24 \mathrm{E}+00$ & 0.76 & 0.45 \\
\hline${ }^{238} \mathrm{U}(\mathrm{IM})$ & $2.36 \mathrm{E}+02$ & $2.32 \mathrm{E}+02$ & $6.44 \mathrm{E}+01$ & 0.98 & 0.28 \\
\hline${ }^{239} \mathrm{Pu}(\mathrm{IM})$ & $2.21 \mathrm{E}+01$ & $1.66 \mathrm{E}+01$ & $6.43 \mathrm{E}+00$ & 0.75 & 0.39 \\
\hline${ }^{240} \mathrm{Pu}(\mathrm{IM})$ & $9.77 \mathrm{E}+03$ & $1.08 \mathrm{E}+04$ & $7.83 \mathrm{E}+03$ & 1.10 & 0.73 \\
\hline
\end{tabular}


Table 3-7. Composition of the Oxalic Acid Leachates from Tank 16H Annulus Sample HTF-16-N-1 from Three Leaching Cycles

\begin{tabular}{|c|c|c|c|c|c|}
\hline Analyte (Method) & $\begin{array}{c}\text { Decant } 1 \\
\text { (mg/L) }\end{array}$ & $\begin{array}{c}\text { Decant } 2 \\
\text { (mg/L) }\end{array}$ & $\begin{array}{c}\text { Decant } 3 \\
(\mathrm{mg} / \mathrm{L})\end{array}$ & $\frac{\text { Decant } 2}{\text { Decant } 1}$ & $\frac{\text { Decant 3 }}{\text { Decant 2 }}$ \\
\hline $\mathrm{F}^{-}(\mathrm{IC})$ & $<1.00 \mathrm{E}+02$ & $<1.00 \mathrm{E}+02$ & $<1.00 \mathrm{E}+02$ & - & - \\
\hline $\mathrm{CH}_{2} \mathrm{O}^{-}$(IC) & $<1.00 \mathrm{E}+02$ & $<1.00 \mathrm{E}+02$ & $<1.00 \mathrm{E}+02$ & - & - \\
\hline $\mathrm{Cl}^{-}$(IC) & $1.97 \mathrm{E}+02$ & $<1.00 \mathrm{E}+02$ & $<1.00 \mathrm{E}+02$ & - & - \\
\hline $\mathrm{NO}_{2}^{-}(\mathrm{IC})$ & $1.09 \mathrm{E}+04$ & $<1.00 \mathrm{E}+02$ & $<1.00 \mathrm{E}+02$ & - & - \\
\hline $\mathrm{Br}^{-}$(IC) & $<1.00 \mathrm{E}+03$ & $<1.00 \mathrm{E}+03$ & $<1.00 \mathrm{E}+03$ & - & - \\
\hline $\mathrm{NO}_{3}^{-}(\mathrm{IC})$ & $1.27 \mathrm{E}+04$ & $8.08 \mathrm{E}+03$ & $4.56 \mathrm{E}+03$ & 0.64 & 0.56 \\
\hline $\mathrm{PO}_{4}^{3-}(\mathrm{IC})$ & $<1.00 \mathrm{E}+02$ & $<1.00 \mathrm{E}+02$ & $<1.00 \mathrm{E}+02$ & - & - \\
\hline $\mathrm{SO}_{4}^{2-}(\mathrm{IC})$ & $<1.20 \mathrm{E}+03$ & $<1.20 \mathrm{E}+03$ & $<1.20 \mathrm{E}+03$ & - & - \\
\hline $\mathrm{C}_{2} \mathrm{O}_{4}{ }^{2-}$ (IC) & $3.59 \mathrm{E}+04$ & $3.46 \mathrm{E}+04$ & $3.82 \mathrm{E}+04$ & 0.96 & 1.10 \\
\hline $\mathrm{Ag}(\mathrm{IE})$ & $<1.46 \mathrm{E}+00$ & $<1.46 \mathrm{E}+00$ & $<1.46 \mathrm{E}+00$ & - & - \\
\hline $\mathrm{Al}$ (IE) & $3.80 \mathrm{E}+03$ & $2.94 \mathrm{E}+03$ & $3.59 \mathrm{E}+03$ & 0.77 & 1.22 \\
\hline B (IE) & $<2.02 \mathrm{E}+00$ & $<2.02 \mathrm{E}+00$ & $<2.02 \mathrm{E}+00$ & - & - \\
\hline $\mathrm{Ba}$ (IE) & $8.90 \mathrm{E}-01$ & $1.41 \mathrm{E}+00$ & $1.82 \mathrm{E}+00$ & 1.58 & 1.29 \\
\hline $\mathrm{Be}$ (IE) & $<6.00 \mathrm{E}-02$ & $<6.00 \mathrm{E}-02$ & $<6.00 \mathrm{E}-02$ & - & - \\
\hline $\mathrm{Ca}$ (IE) & $9.68 \mathrm{E}+00$ & $2.56 \mathrm{E}+01$ & $6.67 \mathrm{E}+01$ & 2.64 & 2.61 \\
\hline $\mathrm{Cd}$ (IE) & $<6.40 \mathrm{E}-01$ & $<6.40 \mathrm{E}-01$ & $<6.40 \mathrm{E}-01$ & - & - \\
\hline $\mathrm{Ce}$ (IE) & $<7.07 \mathrm{E}+00$ & $<7.07 \mathrm{E}+00$ & $<7.07 \mathrm{E}+00$ & - & - \\
\hline Co (IE) & $<9.70 \mathrm{E}-01$ & $<9.70 \mathrm{E}-01$ & $<9.70 \mathrm{E}-01$ & - & - \\
\hline $\mathrm{Cr}$ (IE) & $5.08 \mathrm{E}+00$ & $7.52 \mathrm{E}+00$ & $5.67 \mathrm{E}+00$ & 1.48 & 0.75 \\
\hline $\mathrm{Cu}$ (IE) & $3.78 \mathrm{E}+01$ & $2.74 \mathrm{E}+01$ & $1.48 \mathrm{E}+01$ & 0.72 & 0.54 \\
\hline $\mathrm{Fe}$ (IE) & $3.40 \mathrm{E}+01$ & $7.99 \mathrm{E}+01$ & $1.46 \mathrm{E}+02$ & 2.35 & 1.83 \\
\hline Gd (IE) & $<7.80 \mathrm{E}-01$ & $<7.80 \mathrm{E}-01$ & $<7.80 \mathrm{E}-01$ & - & - \\
\hline K (IE) & $1.08 \mathrm{E}+02$ & $5.28 \mathrm{E}+01$ & $3.36 \mathrm{E}+01$ & 0.49 & 0.64 \\
\hline La (IE) & $<6.70 \mathrm{E}-01$ & $<6.70 \mathrm{E}-01$ & $<6.70 \mathrm{E}-01$ & - & - \\
\hline Li (IE) & $<1.46 \mathrm{E}+00$ & $<1.46 \mathrm{E}+00$ & $<1.46 \mathrm{E}+00$ & - & - \\
\hline $\mathrm{Mg}$ (IE) & $1.78 \mathrm{E}+01$ & $2.22 \mathrm{E}+01$ & $3.77 \mathrm{E}+01$ & 1.25 & 1.70 \\
\hline Mn (IE) & $1.35 \mathrm{E}+00$ & $1.80 \mathrm{E}+00$ & $2.31 \mathrm{E}+00$ & 1.33 & 1.28 \\
\hline Mo (IE) & $<6.17 \mathrm{E}+00$ & $<6.17 \mathrm{E}+00$ & $<6.17 \mathrm{E}+00$ & - & - \\
\hline
\end{tabular}


Table 3-7. Composition of the Oxalic Acid Leachates from Tank 16H Annulus Sample HTF-16-N-1 from Three Leaching Cycles (continued)

\begin{tabular}{|c|c|c|c|c|c|}
\hline Analyte (Method) & $\begin{array}{c}\text { Decant } 1 \\
(\mathrm{mg} / \mathrm{L})\end{array}$ & $\begin{array}{c}\text { Decant } 2 \\
(\mathrm{mg} / \mathrm{L}) \\
\end{array}$ & $\begin{array}{c}\text { Decant } 3 \\
(\mathrm{mg} / \mathrm{L}) \\
\end{array}$ & $\frac{\text { Decant } 2}{\text { Decant } 1}$ & $\frac{\text { Decant } 3}{\text { Decant } 2}$ \\
\hline $\mathrm{Na}$ (IE) & $2.30 \mathrm{E}+04$ & $1.08 \mathrm{E}+04$ & $7.42 \mathrm{E}+03$ & 0.47 & 0.69 \\
\hline Ni (IE) & $<4.55 \mathrm{E}+00$ & $<4.55 \mathrm{E}+00$ & $<4.55 \mathrm{E}+00$ & - & - \\
\hline $\mathrm{P}$ (IE) & $<8.66 \mathrm{E}+00$ & $<8.66 \mathrm{E}+00$ & $<8.66 \mathrm{E}+00$ & - & - \\
\hline $\mathrm{Pb}$ (IE) & $<7.16 \mathrm{E}+00$ & $1.16 \mathrm{E}+01$ & $1.25 \mathrm{E}+01$ & - & - \\
\hline S (IE) & $3.95 \mathrm{E}+02$ & $1.61 \mathrm{E}+02$ & $1.10 \mathrm{E}+02$ & 0.41 & 0.68 \\
\hline $\mathrm{Sb}$ (IE) & $<1.07 \mathrm{E}+01$ & $<1.07 \mathrm{E}+01$ & $<1.07 \mathrm{E}+01$ & - & - \\
\hline Si (IE) & $8.09 \mathrm{E}+01$ & $8.03 \mathrm{E}+02$ & $7.49 \mathrm{E}+02$ & 9.93 & 0.93 \\
\hline Sn (IE) & $<5.61 \mathrm{E}+00$ & $<5.61 \mathrm{E}+00$ & $<5.61 \mathrm{E}+00$ & - & - \\
\hline Sr (IE) & $2.80 \mathrm{E}-01$ & $2.50 \mathrm{E}-01$ & $4.00 \mathrm{E}-01$ & 0.89 & 1.60 \\
\hline Th (IE) & $<2.68 \mathrm{E}+00$ & $<2.68 \mathrm{E}+00$ & $<2.68 \mathrm{E}+00$ & - & - \\
\hline Ti (IE) & $3.40 \mathrm{E}-01$ & $6.10 \mathrm{E}-01$ & $7.60 \mathrm{E}-01$ & - & - \\
\hline U (IE) & $<3.27 \mathrm{E}+01$ & $<3.27 \mathrm{E}+01$ & $<3.27 \mathrm{E}+01$ & - & - \\
\hline V (IE) & $<4.70 \mathrm{E}-01$ & $<4.70 \mathrm{E}-01$ & $<4.70 \mathrm{E}-01$ & - & - \\
\hline Zn (IE) & $7.97 \mathrm{E}+01$ & $3.10 \mathrm{E}+01$ & $3.18 \mathrm{E}+01$ & 0.39 & 1.03 \\
\hline Zr (IE) & $4.50 \mathrm{E}-01$ & $8.30 \mathrm{E}-01$ & $9.10 \mathrm{E}-01$ & 1.84 & 1.10 \\
\hline $\mathrm{Hg}(\mathrm{CV})$ & $1.39 \mathrm{E}+01$ & $8.74 \mathrm{E}+00$ & $1.31 \mathrm{E}+01$ & 0.63 & 1.50 \\
\hline${ }^{232} \mathrm{Th}$ (IM) & $3.00 \mathrm{E}-02$ & $2.91 \mathrm{E}-02$ & $2.60 \mathrm{E}-02$ & 0.97 & 0.89 \\
\hline${ }^{233} \mathrm{U}(\mathrm{IM})$ & $<2.00 \mathrm{E}-02$ & $<1.00 \mathrm{E}-02$ & $<1.00 \mathrm{E}-02$ & - & - \\
\hline${ }^{234} \mathrm{U}(\mathrm{IM})$ & $<2.00 \mathrm{E}-02$ & $1.01 \mathrm{E}-02$ & $<1.00 \mathrm{E}-02$ & - & - \\
\hline${ }^{235} \mathrm{U}(\mathrm{IM})$ & 4.37E-01 & $2.30 \mathrm{E}-01$ & $1.19 \mathrm{E}-01$ & 0.53 & 0.52 \\
\hline${ }^{236} \mathrm{U}(\mathrm{IM})$ & $5.40 \mathrm{E}-02$ & $2.53 \mathrm{E}-02$ & $1.65 \mathrm{E}-02$ & 0.47 & 0.65 \\
\hline${ }^{238} \mathrm{U}$ (IM) & $1.04 \mathrm{E}+01$ & $5.72 \mathrm{E}+00$ & $2.86 \mathrm{E}+00$ & 0.55 & 0.50 \\
\hline U-total (IM) & $1.09 \mathrm{E}+01$ & $5.98 \mathrm{E}+00$ & $3.00 \mathrm{E}+00$ & 0.55 & 0.50 \\
\hline${ }^{137} \mathrm{Cs}$ (GS) & $3.07 \mathrm{E}-01$ & 5.19E-01 & $6.49 \mathrm{E}-01$ & 1.69 & 1.25 \\
\hline${ }^{99} \mathrm{Tc}(\mathrm{IM})$ & $8.38 \mathrm{E}-01$ & 4.34E-01 & $2.53 \mathrm{E}-01$ & 0.52 & 0.58 \\
\hline${ }^{237} \mathrm{~Np}$ (IM) & $1.10 \mathrm{E}-01$ & $6.13 \mathrm{E}-02$ & $3.12 \mathrm{E}-02$ & 0.56 & 0.51 \\
\hline${ }^{239} \mathrm{Pu}$ (IM) & $8.50 \mathrm{E}-02$ & $5.90 \mathrm{E}-02$ & 4.01E-02 & 0.69 & 0.68 \\
\hline${ }^{240} \mathrm{Pu}$ (IM) & $2.25 \mathrm{E}-02$ & $1.15 \mathrm{E}-02$ & $<1.00 \mathrm{E}-02$ & 0.51 & - \\
\hline $\mathrm{pH}$ (pH units) & 6.85 & 1.82 & 1.36 & - & - \\
\hline
\end{tabular}


SRNL-STI-2012-00309

Revision 0

Table 3-7. Composition of the Oxalic Acid Leachates from Tank 16H Annulus Sample HTF-16-N-1 from Three Leaching Cycles (continued)

\begin{tabular}{|l|c|c|c|c|c||}
\hline Analyte (Method) & $\begin{array}{c}\text { Decant 1 } \\
(\mathbf{d p m} / \mathbf{m l})\end{array}$ & $\begin{array}{c}\text { Decant 2 } \\
(\mathbf{d p m} / \mathbf{m l})\end{array}$ & $\begin{array}{c}\text { Decant 3 } \\
(\mathbf{d p m} / \mathbf{m l})\end{array}$ & $\begin{array}{c}\text { Decant 2 } \\
\text { Decant 1 }\end{array}$ & $\begin{array}{c}\text { Decant 3 } \\
\text { Decant 2 }\end{array}$ \\
\hline${ }^{137} \mathrm{Cs}(\mathrm{GS})$ & $5.91 \mathrm{E}+07$ & $1.00 \mathrm{E}+08$ & $1.25 \mathrm{E}+08$ & 1.69 & 1.25 \\
\hline${ }^{99} \mathrm{Tc}(\mathrm{IM})$ & $3.16 \mathrm{E}+04$ & $1.63 \mathrm{E}+04$ & $9.51 \mathrm{E}+03$ & 0.52 & 0.58 \\
\hline${ }^{232} \mathrm{Th}(\mathrm{IM})$ & $7.33 \mathrm{E}-03$ & $7.12 \mathrm{E}-03$ & $6.35 \mathrm{E}-03$ & 0.97 & 0.89 \\
\hline${ }^{233} \mathrm{U}(\mathrm{IM})$ & $<4.28 \mathrm{E}+02$ & $<2.14 \mathrm{E}+02$ & $<2.14 \mathrm{E}+02$ & - & - \\
\hline${ }^{234} \mathrm{U}(\mathrm{IM})$ & $<2.78 \mathrm{E}+02$ & $1.40 \mathrm{E}+02$ & $<1.39 \mathrm{E}+02$ & - & - \\
\hline${ }^{235} \mathrm{U}(\mathrm{IM})$ & $2.10 \mathrm{E}+00$ & $1.10 \mathrm{E}+00$ & $5.70 \mathrm{E}-01$ & 0.53 & 0.52 \\
\hline${ }^{237} \mathrm{~Np}(\mathrm{IM})$ & $7.76 \mathrm{E}+00$ & $3.63 \mathrm{E}+00$ & $2.37 \mathrm{E}+00$ & 0.47 & 0.65 \\
\hline${ }^{238} \mathrm{U}(\mathrm{IM})$ & $1.72 \mathrm{E}+02$ & $9.59 \mathrm{E}+01$ & $4.88 \mathrm{E}+01$ & 0.56 & 0.51 \\
\hline${ }^{239} \mathrm{Pu}(\mathrm{IM})$ & $7.79 \mathrm{E}+00$ & $4.26 \mathrm{E}+00$ & $2.13 \mathrm{E}+00$ & 0.55 & 0.50 \\
\hline${ }^{240} \mathrm{Pu}(\mathrm{IM})$ & $1.16 \mathrm{E}+04$ & $8.03 \mathrm{E}+03$ & $5.45 \mathrm{E}+03$ & 0.69 & 0.68 \\
\hline \hline
\end{tabular}


Table 3-8. Composition of the Oxalic Acid Leachates from Tank 16H Annulus Sample HTF-16-S-2 from Three Leaching Cycles

\begin{tabular}{|c|c|c|c|c|c|}
\hline Analyte (Method) & $\begin{array}{c}\text { Decant } 1 \\
(\mathrm{mg} / \mathrm{L})\end{array}$ & $\begin{array}{c}\text { Decant } 2 \\
(\mathrm{mg} / \mathrm{L})\end{array}$ & $\begin{array}{c}\text { Decant } 3 \\
(\mathrm{mg} / \mathrm{L}) \\
\end{array}$ & $\frac{\text { Decant } 2}{\text { Decant } 1}$ & $\frac{\text { Decant } 3}{\text { Decant } 2}$ \\
\hline $\mathrm{F}^{-}(\mathrm{IC})$ & $<1.00 \mathrm{E}+02$ & $<1.00 \mathrm{E}+02$ & $<1.00 \mathrm{E}+02$ & - & - \\
\hline $\mathrm{CH}_{2} \mathrm{O}^{-}$(IC) & $<1.00 \mathrm{E}+02$ & $<1.00 \mathrm{E}+02$ & $<1.00 \mathrm{E}+02$ & - & - \\
\hline $\mathrm{Cl}^{-}$(IC) & $<1.00 \mathrm{E}+02$ & $<1.00 \mathrm{E}+02$ & $<1.00 \mathrm{E}+02$ & - & - \\
\hline $\mathrm{NO}_{2}^{-}$(IC) & $1.96 \mathrm{E}+04$ & $<1.00 \mathrm{E}+02$ & $<1.00 \mathrm{E}+02$ & - & - \\
\hline $\mathrm{Br}^{-}$(IC) & $<1.00 \mathrm{E}+03$ & $<1.00 \mathrm{E}+03$ & $<1.00 \mathrm{E}+03$ & - & - \\
\hline $\mathrm{NO}_{3}^{-}$(IC) & $1.94 \mathrm{E}+04$ & $1.18 \mathrm{E}+04$ & $4.09 \mathrm{E}+03$ & 0.61 & 0.35 \\
\hline $\mathrm{PO}_{4}^{3-}(\mathrm{IC})$ & $<1.00 \mathrm{E}+02$ & $<1.00 \mathrm{E}+02$ & $<1.00 \mathrm{E}+02$ & - & - \\
\hline $\mathrm{SO}_{4}^{2-}(\mathrm{IC})$ & $<1.20 \mathrm{E}+03$ & $<1.20 \mathrm{E}+03$ & $<1.20 \mathrm{E}+03$ & - & - \\
\hline $\mathrm{C}_{2} \mathrm{O}_{4}{ }^{2-}$ (IC) & $3.59 \mathrm{E}+04$ & $3.70 \mathrm{E}+04$ & $3.69 \mathrm{E}+04$ & 1.03 & 1.00 \\
\hline $\mathrm{Ag}(\mathrm{IE})$ & $<1.46 \mathrm{E}+00$ & $<1.46 \mathrm{E}+00$ & $<1.46 \mathrm{E}+00$ & - & - \\
\hline $\mathrm{Al}$ (IE) & $2.71 \mathrm{E}+03$ & $3.40 \mathrm{E}+03$ & $1.67 \mathrm{E}+03$ & 1.25 & 0.49 \\
\hline B (IE) & $<2.02 \mathrm{E}+00$ & $<2.02 \mathrm{E}+00$ & $<2.02 \mathrm{E}+00$ & - & - \\
\hline $\mathrm{Ba}$ (IE) & $1.22 \mathrm{E}+00$ & $3.50 \mathrm{E}+00$ & $2.57 \mathrm{E}+00$ & 2.87 & 0.73 \\
\hline $\mathrm{Be}$ (IE) & $<6.00 \mathrm{E}-02$ & $<6.00 \mathrm{E}-02$ & $<6.00 \mathrm{E}-02$ & - & - \\
\hline $\mathrm{Ca}$ (IE) & $3.85 \mathrm{E}+00$ & $4.65 \mathrm{E}+00$ & $3.59 \mathrm{E}+01$ & 1.21 & 7.72 \\
\hline $\mathrm{Cd}$ (IE) & $<6.40 \mathrm{E}-01$ & $<6.40 \mathrm{E}-01$ & $<6.40 \mathrm{E}-01$ & - & - \\
\hline $\mathrm{Ce}$ (IE) & $<7.07 \mathrm{E}+00$ & $<7.07 \mathrm{E}+00$ & $<7.07 \mathrm{E}+00$ & - & - \\
\hline Co (IE) & $<9.70 \mathrm{E}-01$ & $<9.70 \mathrm{E}-01$ & $<9.70 \mathrm{E}-01$ & - & - \\
\hline $\mathrm{Cr}$ (IE) & $3.53 \mathrm{E}+00$ & $8.52 \mathrm{E}+00$ & $5.11 \mathrm{E}+00$ & 2.41 & 0.60 \\
\hline $\mathrm{Cu}$ (IE) & $2.25 \mathrm{E}+01$ & $1.59 \mathrm{E}+01$ & $7.94 \mathrm{E}+00$ & 0.71 & 0.50 \\
\hline $\mathrm{Fe}$ (IE) & $3.80 \mathrm{E}+01$ & $1.77 \mathrm{E}+02$ & $1.89 \mathrm{E}+02$ & 4.66 & 1.07 \\
\hline Gd (IE) & $<7.80 \mathrm{E}-01$ & $<7.80 \mathrm{E}-01$ & $<7.80 \mathrm{E}-01$ & - & - \\
\hline K (IE) & $2.12 \mathrm{E}+02$ & $1.12 \mathrm{E}+02$ & $4.06 \mathrm{E}+01$ & 0.53 & 0.36 \\
\hline La (IE) & $<6.70 \mathrm{E}-01$ & $<6.70 \mathrm{E}-01$ & $<6.70 \mathrm{E}-01$ & - & - \\
\hline Li (IE) & $<1.46 \mathrm{E}+00$ & $<1.46 \mathrm{E}+00$ & $<1.46 \mathrm{E}+00$ & - & - \\
\hline Mg (IE) & $2.20 \mathrm{E}+01$ & $3.39 \mathrm{E}+01$ & $4.45 \mathrm{E}+01$ & 1.54 & 1.31 \\
\hline Mn (IE) & $2.20 \mathrm{E}+00$ & $4.41 \mathrm{E}+00$ & $3.95 \mathrm{E}+00$ & 2.00 & 0.90 \\
\hline Mo (IE) & $<6.17 \mathrm{E}+00$ & $<6.17 \mathrm{E}+00$ & $<6.17 \mathrm{E}+00$ & - & - \\
\hline
\end{tabular}


Table 3-8. Composition of the Oxalic Acid Leachates from Tank 16H Annulus Sample HTF-16-S-2 from Three Leaching Cycles (continued)

\begin{tabular}{|c|c|c|c|c|c|}
\hline Analyte (Method) & $\begin{array}{c}\text { Decant } 1 \\
(\mathrm{mg} / \mathrm{L})\end{array}$ & $\begin{array}{c}\text { Decant } 2 \\
(\mathrm{mg} / \mathrm{L}) \\
\end{array}$ & $\begin{array}{c}\text { Decant } 3 \\
(\mathrm{mg} / \mathrm{L}) \\
\end{array}$ & $\frac{\text { Decant } 2}{\text { Decant } 1}$ & $\frac{\text { Decant } 3}{\text { Decant } 2}$ \\
\hline $\mathrm{Na}$ (IE) & $2.94 \mathrm{E}+04$ & $1.40 \mathrm{E}+04$ & $6.05 \mathrm{E}+03$ & 0.48 & 0.43 \\
\hline Ni (IE) & $<4.55 \mathrm{E}+00$ & $<4.55 \mathrm{E}+00$ & $<4.55 \mathrm{E}+00$ & - & - \\
\hline $\mathrm{P}$ (IE) & $<8.66 \mathrm{E}+00$ & $<8.66 \mathrm{E}+00$ & $<8.66 \mathrm{E}+00$ & - & - \\
\hline $\mathrm{Pb}$ (IE) & $9.43 \mathrm{E}+00$ & $2.19 \mathrm{E}+01$ & $1.99 \mathrm{E}+01$ & - & - \\
\hline S (IE) & $5.50 \mathrm{E}+02$ & $2.44 \mathrm{E}+02$ & $1.13 \mathrm{E}+02$ & 0.44 & 0.46 \\
\hline $\mathrm{Sb}$ (IE) & $<1.07 \mathrm{E}+01$ & $<1.07 \mathrm{E}+01$ & $<1.07 \mathrm{E}+01$ & - & - \\
\hline Si (IE) & $5.86 \mathrm{E}+01$ & $1.67 \mathrm{E}+03$ & $3.83 \mathrm{E}+02$ & 28.5 & 0.23 \\
\hline Sn (IE) & $<5.61 \mathrm{E}+00$ & $<5.61 \mathrm{E}+00$ & $<5.61 \mathrm{E}+00$ & - & - \\
\hline Sr (IE) & $2.10 \mathrm{E}-01$ & $6.10 \mathrm{E}-01$ & $6.20 \mathrm{E}-01$ & 2.90 & 1.02 \\
\hline Th (IE) & $<2.68 \mathrm{E}+00$ & $<2.68 \mathrm{E}+00$ & $<2.68 \mathrm{E}+00$ & - & - \\
\hline Ti (IE) & $2.40 \mathrm{E}-01$ & $7.10 \mathrm{E}-01$ & $7.50 \mathrm{E}-01$ & 2.96 & 1.06 \\
\hline U (IE) & $<3.27 \mathrm{E}+01$ & $<3.27 \mathrm{E}+01$ & $<3.27 \mathrm{E}+01$ & - & - \\
\hline V (IE) & $<4.70 \mathrm{E}-01$ & $<4.70 \mathrm{E}-01$ & $<4.70 \mathrm{E}-01$ & - & - \\
\hline Zn (IE) & $1.42 \mathrm{E}+01$ & $1.81 \mathrm{E}+01$ & $1.12 \mathrm{E}+01$ & 1.27 & 0.62 \\
\hline Zr (IE) & $1.15 \mathrm{E}+00$ & $3.32 \mathrm{E}+00$ & $3.32 \mathrm{E}+00$ & 2.89 & 1.00 \\
\hline $\mathrm{Hg}(\mathrm{CV})$ & $1.13 \mathrm{E}+01$ & $5.80 \mathrm{E}+00$ & $5.29 \mathrm{E}+00$ & 0.51 & 0.91 \\
\hline${ }^{232} \mathrm{Th}$ (IM) & $3.00 \mathrm{E}-02$ & $3.00 \mathrm{E}-02$ & $2.12 \mathrm{E}-02$ & 1.00 & 0.71 \\
\hline${ }^{233} \mathrm{U}(\mathrm{IM})$ & $<2.00 \mathrm{E}-02$ & $<2.00 \mathrm{E}-02$ & $<1.00 \mathrm{E}-02$ & - & - \\
\hline${ }^{234} \mathrm{U}(\mathrm{IM})$ & $2.12 \mathrm{E}-02$ & $<2.00 \mathrm{E}-02$ & $<1.00 \mathrm{E}-02$ & - & - \\
\hline${ }^{235} \mathrm{U}(\mathrm{IM})$ & $6.65 \mathrm{E}-01$ & $4.50 \mathrm{E}-01$ & $1.65 \mathrm{E}-01$ & 0.68 & 0.37 \\
\hline${ }^{236} \mathrm{U}(\mathrm{IM})$ & $6.34 \mathrm{E}-02$ & $3.64 \mathrm{E}-02$ & $1.52 \mathrm{E}-02$ & 0.57 & 0.42 \\
\hline${ }^{238} \mathrm{U}$ (IM) & $1.62 \mathrm{E}+01$ & $1.16 \mathrm{E}+01$ & $4.35 \mathrm{E}+00$ & 0.72 & 0.38 \\
\hline U-total (IM) & $1.69 \mathrm{E}+01$ & $1.21 \mathrm{E}+01$ & $4.53 \mathrm{E}+00$ & 0.71 & 0.38 \\
\hline${ }^{137} \mathrm{Cs}$ (GS) & 5.19E-01 & $7.48 \mathrm{E}-01$ & $3.96 \mathrm{E}-01$ & 1.44 & 0.53 \\
\hline${ }^{99} \mathrm{Tc}(\mathrm{IM})$ & $1.75 \mathrm{E}+00$ & $7.48 \mathrm{E}-01$ & $2.65 \mathrm{E}-01$ & 0.43 & 0.35 \\
\hline${ }^{237} \mathrm{~Np}$ (IM) & $1.62 \mathrm{E}-01$ & $1.04 \mathrm{E}-01$ & 4.91E-02 & 0.64 & 0.47 \\
\hline${ }^{239} \mathrm{Pu}$ (IM) & $1.05 \mathrm{E}-01$ & $1.16 \mathrm{E}-01$ & $6.87 \mathrm{E}-02$ & 1.10 & 0.59 \\
\hline${ }^{240} \mathrm{Pu}$ (IM) & $<2.00 \mathrm{E}-02$ & $<2.00 \mathrm{E}-02$ & $1.14 \mathrm{E}-02$ & - & - \\
\hline $\mathrm{pH}$ (pH units) & 7.23 & 2.90 & 1.26 & - & - \\
\hline
\end{tabular}


Table 3-8. Composition of the Oxalic Acid Leachates from Tank 16H Annulus Sample HTF-16-S-2 from Three Leaching Cycles (continued)

\begin{tabular}{|l|c|c|c|c|c||}
\hline Analyte (Method) & $\begin{array}{c}\text { Decant 1 } \\
(\mathbf{d p m} / \mathbf{m l})\end{array}$ & $\begin{array}{c}\text { Decant 2 } \\
(\mathbf{d p m} / \mathbf{m l})\end{array}$ & $\begin{array}{c}\text { Decant 3 } \\
(\mathbf{d p m} / \mathbf{m l})\end{array}$ & $\begin{array}{c}\text { Decant 2 } \\
\text { Decant 1 }\end{array}$ & $\begin{array}{c}\text { Decant 3 } \\
\text { Decant 2 }\end{array}$ \\
\hline${ }^{137} \mathrm{Cs}(\mathrm{GS})$ & $9.99 \mathrm{E}+07$ & $1.44 \mathrm{E}+08$ & $7.62 \mathrm{E}+07$ & 1.44 & 0.53 \\
\hline${ }^{99} \mathrm{Tc}(\mathrm{IM})$ & $6.60 \mathrm{E}+04$ & $2.82 \mathrm{E}+04$ & $9.98 \mathrm{E}+03$ & 0.43 & 0.35 \\
\hline${ }^{232} \mathrm{Th}(\mathrm{IM})$ & $7.33 \mathrm{E}-03$ & $7.33 \mathrm{E}-03$ & $5.17 \mathrm{E}-03$ & 1.00 & 0.71 \\
\hline${ }^{233} \mathrm{U}(\mathrm{IM})$ & $<4.28 \mathrm{E}+02$ & $<4.28 \mathrm{E}+02$ & $<2.14 \mathrm{E}+02$ & - & - \\
\hline${ }^{234} \mathrm{U}(\mathrm{IM})$ & $2.95 \mathrm{E}+02$ & $<2.78 \mathrm{E}+02$ & $<1.39 \mathrm{E}+02$ & - & - \\
\hline${ }^{235} \mathrm{U}(\mathrm{IM})$ & $3.19 \mathrm{E}+00$ & $2.16 \mathrm{E}+00$ & $7.93 \mathrm{E}-01$ & 0.68 & 0.37 \\
\hline${ }^{237} \mathrm{~Np}(\mathrm{IM})$ & $9.11 \mathrm{E}+00$ & $5.23 \mathrm{E}+00$ & $2.18 \mathrm{E}+00$ & 0.57 & 0.42 \\
\hline${ }^{238} \mathrm{U}(\mathrm{IM})$ & $2.53 \mathrm{E}+02$ & $1.62 \mathrm{E}+02$ & $7.69 \mathrm{E}+01$ & 0.64 & 0.47 \\
\hline${ }^{239} \mathrm{Pu}(\mathrm{IM})$ & $1.20 \mathrm{E}+01$ & $8.65 \mathrm{E}+00$ & $3.24 \mathrm{E}+00$ & 0.72 & 0.38 \\
\hline${ }^{240} \mathrm{Pu}(\mathrm{IM})$ & $1.43 \mathrm{E}+04$ & $1.57 \mathrm{E}+04$ & $9.36 \mathrm{E}+03$ & 1.10 & 0.59 \\
\hline \hline
\end{tabular}


Table 3-9. Composition of the Oxalic Acid Leachates from Tank 16H Annulus Sample HTF-16-W-1 from Three Leaching Cycles

\begin{tabular}{|c|c|c|c|c|c|}
\hline Analyte (Method) & $\begin{array}{c}\text { Decant } 1 \\
\text { (mg/L) }\end{array}$ & $\begin{array}{c}\text { Decant } 2 \\
\text { (mg/L) }\end{array}$ & $\begin{array}{c}\text { Decant } 3 \\
(\mathrm{mg} / \mathrm{L})\end{array}$ & $\frac{\text { Decant } 2}{\text { Decant } 1}$ & $\frac{\text { Decant 3 }}{\text { Decant 2 }}$ \\
\hline $\mathrm{F}^{-}(\mathrm{IC})$ & $<1.00 \mathrm{E}+02$ & $<1.00 \mathrm{E}+02$ & $<1.00 \mathrm{E}+02$ & - & - \\
\hline $\mathrm{CH}_{2} \mathrm{O}^{-}$(IC) & $<1.00 \mathrm{E}+02$ & $<1.00 \mathrm{E}+02$ & $<1.00 \mathrm{E}+02$ & - & - \\
\hline $\mathrm{Cl}^{-}$(IC) & $1.64 \mathrm{E}+02$ & $<1.00 \mathrm{E}+02$ & $<1.00 \mathrm{E}+02$ & - & - \\
\hline $\mathrm{NO}_{2}^{-}(\mathrm{IC})$ & $1.92 \mathrm{E}+04$ & $<1.00 \mathrm{E}+02$ & $<1.00 \mathrm{E}+02$ & - & - \\
\hline $\mathrm{Br}^{-}$(IC) & $<1.00 \mathrm{E}+03$ & $<1.00 \mathrm{E}+03$ & $<1.00 \mathrm{E}+03$ & - & - \\
\hline $\mathrm{NO}_{3}^{-}(\mathrm{IC})$ & $1.84 \mathrm{E}+04$ & $1.23 \mathrm{E}+04$ & $5.09 \mathrm{E}+03$ & 0.67 & 0.41 \\
\hline $\mathrm{PO}_{4}^{3-}(\mathrm{IC})$ & $<1.00 \mathrm{E}+02$ & $<1.00 \mathrm{E}+02$ & $<1.00 \mathrm{E}+02$ & - & - \\
\hline $\mathrm{SO}_{4}^{2-}(\mathrm{IC})$ & $<1.20 \mathrm{E}+03$ & $<1.20 \mathrm{E}+03$ & $<1.20 \mathrm{E}+03$ & - & - \\
\hline $\mathrm{C}_{2} \mathrm{O}_{4}{ }^{2-}$ (IC) & $3.63 \mathrm{E}+04$ & $3.60 \mathrm{E}+04$ & $3.63 \mathrm{E}+04$ & 0.99 & 1.01 \\
\hline $\mathrm{Ag}(\mathrm{IE})$ & $<1.46 \mathrm{E}+00$ & $<1.46 \mathrm{E}+00$ & $<1.46 \mathrm{E}+00$ & - & - \\
\hline $\mathrm{Al}$ (IE) & $3.77 \mathrm{E}+03$ & $3.67 \mathrm{E}+03$ & $2.33 \mathrm{E}+03$ & 0.97 & 0.63 \\
\hline B (IE) & $<2.02 \mathrm{E}+00$ & $<2.02 \mathrm{E}+00$ & $<2.02 \mathrm{E}+00$ & - & - \\
\hline $\mathrm{Ba}$ (IE) & $2.62 \mathrm{E}+00$ & $3.05 \mathrm{E}+00$ & $2.55 \mathrm{E}+00$ & 1.16 & 0.84 \\
\hline $\mathrm{Be}$ (IE) & $<6.00 \mathrm{E}-02$ & $<6.00 \mathrm{E}-02$ & $<6.00 \mathrm{E}-02$ & - & - \\
\hline $\mathrm{Ca}$ (IE) & $2.73 \mathrm{E}+00$ & $9.60 \mathrm{E}+00$ & $2.50 \mathrm{E}+01$ & 3.52 & 2.60 \\
\hline $\mathrm{Cd}$ (IE) & $<6.40 \mathrm{E}-01$ & $<6.40 \mathrm{E}-01$ & $<6.40 \mathrm{E}-01$ & - & - \\
\hline $\mathrm{Ce}$ (IE) & $<7.07 \mathrm{E}+00$ & $<7.07 \mathrm{E}+00$ & $<7.07 \mathrm{E}+00$ & - & - \\
\hline Co (IE) & $<9.70 \mathrm{E}-01$ & $<9.70 \mathrm{E}-01$ & $<9.70 \mathrm{E}-01$ & - & - \\
\hline $\mathrm{Cr}$ (IE) & $3.42 \mathrm{E}+00$ & $4.76 \mathrm{E}+00$ & $3.12 \mathrm{E}+00$ & 1.39 & 0.66 \\
\hline $\mathrm{Cu}$ (IE) & $2.91 \mathrm{E}+00$ & $3.56 \mathrm{E}+00$ & $2.29 \mathrm{E}+00$ & 1.22 & 0.64 \\
\hline $\mathrm{Fe}$ (IE) & $3.82 \mathrm{E}+01$ & $1.29 \mathrm{E}+02$ & $1.44 \mathrm{E}+02$ & 3.38 & 1.12 \\
\hline Gd (IE) & $<7.80 \mathrm{E}-01$ & $<7.80 \mathrm{E}-01$ & $<7.80 \mathrm{E}-01$ & - & - \\
\hline K (IE) & $2.90 \mathrm{E}+02$ & $1.54 \mathrm{E}+02$ & $6.74 \mathrm{E}+01$ & 0.53 & 0.44 \\
\hline La (IE) & $7.20 \mathrm{E}-01$ & $<6.70 \mathrm{E}-01$ & $1.78 \mathrm{E}+00$ & - & - \\
\hline Li (IE) & $<1.46 \mathrm{E}+00$ & $<1.46 \mathrm{E}+00$ & $<1.46 \mathrm{E}+00$ & - & - \\
\hline Mg (IE) & $1.25 \mathrm{E}+01$ & $1.12 \mathrm{E}+01$ & $6.78 \mathrm{E}+00$ & 0.90 & 0.61 \\
\hline Mn (IE) & $3.35 \mathrm{E}+00$ & $4.32 \mathrm{E}+00$ & $3.89 \mathrm{E}+00$ & 1.29 & 0.90 \\
\hline Mo (IE) & $<6.17 \mathrm{E}+00$ & $<6.17 \mathrm{E}+00$ & $<6.17 \mathrm{E}+00$ & - & - \\
\hline
\end{tabular}


Table 3-9. Composition of the Oxalic Acid Leachates from Tank 16H Annulus Sample HTF-16-W-1 from Three Leaching Cycles (continued)

\begin{tabular}{|c|c|c|c|c|c|}
\hline Analyte (Method) & $\begin{array}{c}\text { Decant } 1 \\
(\mathrm{mg} / \mathrm{L})\end{array}$ & $\begin{array}{c}\text { Decant } 2 \\
(\mathrm{mg} / \mathrm{L}) \\
\end{array}$ & $\begin{array}{c}\text { Decant } 3 \\
(\mathrm{mg} / \mathrm{L}) \\
\end{array}$ & $\frac{\text { Decant } 2}{\text { Decant } 1}$ & $\frac{\text { Decant } 3}{\text { Decant } 2}$ \\
\hline $\mathrm{Na}$ (IE) & $2.65 \mathrm{E}+04$ & $1.39 \mathrm{E}+04$ & $7.32 \mathrm{E}+03$ & 0.52 & 0.53 \\
\hline Ni (IE) & $<4.55 \mathrm{E}+00$ & $<4.55 \mathrm{E}+00$ & $<4.55 \mathrm{E}+00$ & - & - \\
\hline $\mathrm{P}$ (IE) & $<8.66 \mathrm{E}+00$ & $<8.66 \mathrm{E}+00$ & $<8.66 \mathrm{E}+00$ & - & - \\
\hline $\mathrm{Pb}$ (IE) & $<7.16 \mathrm{E}+00$ & $<7.16 \mathrm{E}+00$ & $9.59 \mathrm{E}+00$ & - & - \\
\hline S (IE) & $3.80 \mathrm{E}+02$ & $1.82 \mathrm{E}+02$ & $1.38 \mathrm{E}+02$ & 0.48 & 0.76 \\
\hline $\mathrm{Sb}$ (IE) & $<1.07 \mathrm{E}+01$ & $<1.07 \mathrm{E}+01$ & $<1.07 \mathrm{E}+01$ & - & - \\
\hline Si (IE) & $7.21 \mathrm{E}+01$ & $1.25 \mathrm{E}+03$ & $3.71 \mathrm{E}+02$ & 17.34 & 0.30 \\
\hline Sn (IE) & $<5.61 \mathrm{E}+00$ & $<5.61 \mathrm{E}+00$ & $<5.61 \mathrm{E}+00$ & - & - \\
\hline Sr (IE) & $1.96 \mathrm{E}+00$ & $1.43 \mathrm{E}+00$ & $1.19 \mathrm{E}+00$ & 0.73 & 0.83 \\
\hline Th (IE) & $<2.68 \mathrm{E}+00$ & $<2.68 \mathrm{E}+00$ & $<2.68 \mathrm{E}+00$ & - & - \\
\hline Ti (IE) & $<1.30 \mathrm{E}-01$ & $1.50 \mathrm{E}-01$ & $3.20 \mathrm{E}-01$ & - & - \\
\hline $\mathrm{U}(\mathrm{IE})$ & $<3.27 \mathrm{E}+01$ & $<3.27 \mathrm{E}+01$ & $<3.27 \mathrm{E}+01$ & - & - \\
\hline V (IE) & $<4.70 \mathrm{E}-01$ & $<4.70 \mathrm{E}-01$ & $<4.70 \mathrm{E}-01$ & - & - \\
\hline Zn (IE) & $1.71 \mathrm{E}+01$ & $1.43 \mathrm{E}+01$ & $8.14 \mathrm{E}+00$ & 0.84 & 0.57 \\
\hline Zr (IE) & $2.01 \mathrm{E}+00$ & $3.46 \mathrm{E}+00$ & $3.80 \mathrm{E}+00$ & 1.72 & 1.10 \\
\hline $\mathrm{Hg}(\mathrm{CV})$ & $1.78 \mathrm{E}+01$ & $1.11 \mathrm{E}+01$ & $1.05 \mathrm{E}+01$ & 0.62 & 0.95 \\
\hline${ }^{232} \mathrm{Th}$ (IM) & $7.50 \mathrm{E}-02$ & $7.50 \mathrm{E}-02$ & $3.00 \mathrm{E}-02$ & 1.00 & 0.40 \\
\hline${ }^{233} \mathrm{U}(\mathrm{IM})$ & $<5.00 \mathrm{E}-02$ & $<5.00 \mathrm{E}-02$ & $<2.00 \mathrm{E}-02$ & - & - \\
\hline${ }^{234} \mathrm{U}(\mathrm{IM})$ & $<5.00 \mathrm{E}-02$ & $<5.00 \mathrm{E}-02$ & $<2.00 \mathrm{E}-02$ & - & - \\
\hline${ }^{235} \mathrm{U}(\mathrm{IM})$ & $1.00 \mathrm{E}+00$ & $7.85 \mathrm{E}-01$ & $3.27 \mathrm{E}-01$ & 0.78 & 0.42 \\
\hline${ }^{236} \mathrm{U}(\mathrm{IM})$ & 7.98E-02 & $6.92 \mathrm{E}-02$ & $3.22 \mathrm{E}-02$ & 0.87 & 0.47 \\
\hline${ }^{238} \mathrm{U}$ (IM) & $2.48 \mathrm{E}+01$ & $1.97 \mathrm{E}+01$ & $8.32 \mathrm{E}+00$ & 0.79 & 0.42 \\
\hline U-total (IM) & $2.59 \mathrm{E}+01$ & $2.05 \mathrm{E}+01$ & $8.67 \mathrm{E}+00$ & 0.79 & 0.42 \\
\hline${ }^{137} \mathrm{Cs}$ (GS) & 4.42E-01 & $6.85 \mathrm{E}-01$ & $4.45 \mathrm{E}-01$ & 1.55 & 0.65 \\
\hline${ }^{99} \mathrm{Tc}(\mathrm{IM})$ & $2.44 \mathrm{E}+00$ & $9.88 \mathrm{E}-01$ & $4.08 \mathrm{E}-01$ & 0.41 & 0.41 \\
\hline${ }^{237} \mathrm{~Np}$ (IM) & $2.05 \mathrm{E}-01$ & $1.71 \mathrm{E}-01$ & $9.23 \mathrm{E}-02$ & 0.83 & 0.54 \\
\hline${ }^{239} \mathrm{Pu}$ (IM) & $1.60 \mathrm{E}-01$ & $1.41 \mathrm{E}-01$ & $8.51 \mathrm{E}-02$ & 0.88 & 0.60 \\
\hline${ }^{240} \mathrm{Pu}$ (IM) & $<5.00 \mathrm{E}-02$ & $<5.00 \mathrm{E}-02$ & $<2.00 \mathrm{E}-02$ & - & - \\
\hline $\mathrm{pH}$ (pH units) & 6.43 & 2.71 & 1.30 & - & - \\
\hline
\end{tabular}


Table 3-9. Composition of the Oxalic Acid Leachates from Tank 16H Annulus Sample HTF-16-W-1 from Three Leaching Cycles (continued)

\begin{tabular}{|l|c|c|c|c|c||}
\hline Analyte (Method) & $\begin{array}{c}\text { Decant 1 } \\
(\mathbf{d p m} / \mathbf{m l})\end{array}$ & $\begin{array}{c}\text { Decant 2 } \\
(\mathbf{d p m} / \mathbf{m l})\end{array}$ & $\begin{array}{c}\text { Decant 3 } \\
(\mathbf{d p m} / \mathbf{m l})\end{array}$ & $\begin{array}{c}\text { Decant 2 } \\
\text { Decant 1 }\end{array}$ & $\begin{array}{c}\text { Decant 3 } \\
\text { Decant 2 }\end{array}$ \\
\hline${ }^{137} \mathrm{Cs}(\mathrm{GS})$ & $8.52 \mathrm{E}+07$ & $1.32 \mathrm{E}+08$ & $8.57 \mathrm{E}+07$ & 1.55 & 0.65 \\
\hline${ }^{99} \mathrm{Tc}(\mathrm{IM})$ & $9.18 \mathrm{E}+04$ & $3.72 \mathrm{E}+04$ & $1.54 \mathrm{E}+04$ & 0.41 & 0.41 \\
\hline${ }^{232} \mathrm{Th}(\mathrm{IM})$ & $1.83 \mathrm{E}-02$ & $1.83 \mathrm{E}-02$ & $7.33 \mathrm{E}-03$ & 1.00 & 0.40 \\
\hline${ }^{233} \mathrm{U}(\mathrm{IM})$ & $<1.07 \mathrm{E}+03$ & $<1.07 \mathrm{E}+03$ & $<4.28 \mathrm{E}+02$ & - & - \\
\hline${ }^{234} \mathrm{U}(\mathrm{IM})$ & $<6.95 \mathrm{E}+02$ & $<6.95 \mathrm{E}+02$ & $<2.78 \mathrm{E}+02$ & - & - \\
\hline${ }^{235} \mathrm{U}(\mathrm{IM})$ & $4.80 \mathrm{E}+00$ & $3.76 \mathrm{E}+00$ & $1.57 \mathrm{E}+00$ & 0.78 & 0.42 \\
\hline${ }^{237} \mathrm{~Np}(\mathrm{IM})$ & $1.15 \mathrm{E}+01$ & $9.93 \mathrm{E}+00$ & $4.62 \mathrm{E}+00$ & 0.87 & 0.47 \\
\hline${ }^{238} \mathrm{U}(\mathrm{IM})$ & $3.21 \mathrm{E}+02$ & $2.67 \mathrm{E}+02$ & $1.44 \mathrm{E}+02$ & 0.83 & 0.54 \\
\hline${ }^{239} \mathrm{Pu}(\mathrm{IM})$ & $1.85 \mathrm{E}+01$ & $1.47 \mathrm{E}+01$ & $6.20 \mathrm{E}+00$ & 0.79 & 0.42 \\
\hline${ }^{240} \mathrm{Pu}(\mathrm{IM})$ & $2.17 \mathrm{E}+04$ & $1.92 \mathrm{E}+04$ & $1.16 \mathrm{E}+04$ & 0.88 & 0.60 \\
\hline \hline
\end{tabular}


SRNL-STI-2012-00309

Revision 0

Table 3-10. Composition of the Oxalic Acid Leached Solids from Tank 16H Annulus Sample HTF-16-E-2

\begin{tabular}{|c|c|c|c|c|c|c|}
\hline Analyte (Method) & $\begin{array}{c}\text { Aqua Regia } \\
\text { Replicate } 1 \\
\text { (mg/kg) }\end{array}$ & $\begin{array}{c}\text { Aqua Regia } \\
\text { Replicate } 2 \\
\text { (mg/kg) }\end{array}$ & $\begin{array}{c}\text { Per Fusion } \\
\text { Replicate } 1 \\
\text { (mg/kg) }\end{array}$ & $\begin{array}{c}\text { Per Fusion } \\
\text { Replicate } 2 \\
\text { (mg/kg) }\end{array}$ & $\begin{array}{c}\text { Average } \\
\text { (mg/kg) }\end{array}$ & $\%$ RSD \\
\hline $\mathrm{Ag}(\mathrm{IE})$ & $<1.13 \mathrm{E}+02$ & $<1.10 \mathrm{E}+02$ & $<1.04 E+03$ & $<9.63 E+02$ & $<1.12 \mathrm{E}+02$ & - \\
\hline $\mathrm{Al}$ (IE) & $6.32 \mathrm{E}+04$ & $6.42 \mathrm{E}+04$ & $7.47 \mathrm{E}+04$ & $7.08 \mathrm{E}+04$ & $6.82 \mathrm{E}+04$ & $8.0 \%$ \\
\hline B (IE) & $<1.58 \mathrm{E}+01$ & $<1.54 \mathrm{E}+01$ & $<1.46 E+02$ & $<1.35 E+02$ & $<1.56 \mathrm{E}+01$ & - \\
\hline $\mathrm{Ba}$ (IE) & $5.51 \mathrm{E}+01$ & $6.60 \mathrm{E}+01$ & $1.38 E+02$ & $7.29 E+01$ & $6.06 \mathrm{E}+01$ & $13 \%$ \\
\hline Be (IE) & $<4.71 \mathrm{E}-01$ & $<4.58 \mathrm{E}-01$ & $<5.78 E+00$ & $<5.35 E+00$ & $<4.65 \mathrm{E}-01$ & - \\
\hline $\mathrm{Ca}$ (IE) & $1.41 \mathrm{E}+04$ & $1.74 \mathrm{E}+04$ & $1.93 E+04$ & $1.12 E+04$ & $1.58 \mathrm{E}+04$ & $15 \%$ \\
\hline Cd (IE) & $3.14 \mathrm{E}+00$ & $3.59 \mathrm{E}+00$ & $<2.24 E+01$ & $<2.07 E+01$ & $3.37 \mathrm{E}+00$ & $9.5 \%$ \\
\hline Ce (IE) & $6.43 \mathrm{E}+01$ & $<5.40 E+01$ & $<5.10 E+02$ & $<4.73 E+02$ & $6.43 \mathrm{E}+01$ & - \\
\hline Co (IE) & $<7.61 \mathrm{E}+00$ & $<7.40 \mathrm{E}+00$ & $<6.14 E+01$ & $<5.69 E+01$ & $<7.51 \mathrm{E}+00$ & - \\
\hline Cr (IE) & $2.62 \mathrm{E}+02$ & $2.49 \mathrm{E}+02$ & $3.15 \mathrm{E}+02$ & $2.27 \mathrm{E}+02$ & $2.63 \mathrm{E}+02$ & $14 \%$ \\
\hline $\mathrm{Cu}$ (IE) & $7.77 \mathrm{E}+01$ & $7.47 \mathrm{E}+01$ & $1.00 \mathrm{E}+02$ & $1.02 \mathrm{E}+02$ & $8.86 \mathrm{E}+01$ & $16 \%$ \\
\hline $\mathrm{Fe}$ (IE) & $3.22 \mathrm{E}+04$ & $2.54 \mathrm{E}+04$ & $4.01 \mathrm{E}+04$ & $4.12 \mathrm{E}+04$ & $3.47 \mathrm{E}+04$ & $21 \%$ \\
\hline Gd (IE) & $<1.69 \mathrm{E}+01$ & $<1.64 \mathrm{E}+01$ & $<1.55 E+02$ & $<1.44 E+02$ & $<1.67 \mathrm{E}+01$ & - \\
\hline K (IE) & $8.93 \mathrm{E}+02$ & $8.24 \mathrm{E}+02$ & $3.50 E+03$ & $3.93 E+03$ & $8.59 \mathrm{E}+02$ & $5.7 \%$ \\
\hline La (IE) & $2.22 \mathrm{E}+01$ & $2.47 \mathrm{E}+01$ & $7.51 \mathrm{E}+01$ & $<4.48 E+01$ & $4.07 \mathrm{E}+01$ & $73 \%$ \\
\hline Li (IE) & $<1.15 \mathrm{E}+01$ & $<1.11 \mathrm{E}+01$ & $<1.05 E+02$ & $<9.77 E+01$ & $<1.13 \mathrm{E}+01$ & - \\
\hline $\mathrm{Mg}$ (IE) & $5.72 \mathrm{E}+02$ & $8.10 \mathrm{E}+02$ & $1.15 \mathrm{E}+03$ & $2.10 \mathrm{E}+03$ & $1.16 \mathrm{E}+03$ & $58 \%$ \\
\hline Mn (IE) & $2.25 \mathrm{E}+02$ & $2.10 \mathrm{E}+02$ & $3.00 \mathrm{E}+02$ & $8.26 \mathrm{E}+02$ & $3.90 \mathrm{E}+02$ & $75 \%$ \\
\hline Mo (IE) & $<4.84 \mathrm{E}+01$ & $<4.71 \mathrm{E}+01$ & $<1.94 E+02$ & $<1.79 E+02$ & $<4.78 \mathrm{E}+01$ & - \\
\hline $\mathrm{Na}$ (IE) & $4.90 \mathrm{E}+04$ & $4.66 \mathrm{E}+04$ & - & - & $4.78 \mathrm{E}+04$ & $3.6 \%$ \\
\hline Ni (IE) & $4.70 \mathrm{E}+01$ & $6.30 \mathrm{E}+01$ & $<3.29 E+02$ & $<3.04 E+02$ & $5.50 \mathrm{E}+01$ & $21 \%$ \\
\hline P (IE) & $9.20 \mathrm{E}+01$ & $1.06 \mathrm{E}+02$ & $<1.18 E+03$ & $<1.10 E+03$ & $9.90 \mathrm{E}+01$ & $10 \%$ \\
\hline $\mathrm{Pb}$ (IE) & $3.06 \mathrm{E}+02$ & $3.20 \mathrm{E}+02$ & $<5.17 E+02$ & $<4.79 E+02$ & $3.13 \mathrm{E}+02$ & $3.2 \%$ \\
\hline S (IE) & $<5.88 E+02$ & $6.30 \mathrm{E}+02$ & $<5.42 E+03$ & $<5.02 E+03$ & $6.30 \mathrm{E}+02$ & - \\
\hline $\mathrm{Sb}$ (IE) & $<1.00 \mathrm{E}+02$ & $<9.75 \mathrm{E}+01$ & $<7.70 E+02$ & $<7.13 E+02$ & $<9.88 \mathrm{E}+01$ & - \\
\hline Si (IE) & - & - & $2.27 \mathrm{E}+05$ & $2.43 \mathrm{E}+05$ & $2.35 \mathrm{E}+05$ & $4.8 \%$ \\
\hline Sn (IE) & $<4.40 \mathrm{E}+01$ & $<4.28 \mathrm{E}+01$ & $<4.05 E+02$ & $<3.75 E+02$ & $<4.34 \mathrm{E}+01$ & - \\
\hline
\end{tabular}

Values in italics were not used in calculating the average. Divide $\mathrm{mg} / \mathrm{kg}$ values by $1 \mathrm{E}+04$ to convert to wt $\%$ basis. 
SRNL-STI-2012-00309

Revision 0

Table 3-10. Composition of the Oxalic Acid Leached Solids from Tank 16H Annulus Sample HTF-16-E-2 (continued)

\begin{tabular}{|c|c|c|c|c|c|c|}
\hline Analyte (Method) & $\begin{array}{c}\text { Aqua Regia } \\
\text { Replicate } 1 \\
\text { (mg/kg) }\end{array}$ & $\begin{array}{c}\text { Aqua Regia } \\
\text { Replicate } 2 \\
\text { (mg/kg) }\end{array}$ & $\begin{array}{c}\text { Per Fusion } \\
\text { Replicate } 1 \\
\text { (mg/kg) }\end{array}$ & $\begin{array}{c}\text { Per Fusion } \\
\text { Replicate } 2 \\
\text { (mg/kg) }\end{array}$ & $\begin{array}{l}\text { Average } \\
\text { (mg/kg) }\end{array}$ & $\%$ RSD \\
\hline $\operatorname{Sr}(\mathrm{IE})$ & $4.66 \mathrm{E}+01$ & $5.15 \mathrm{E}+01$ & $1.06 E+02$ & $6.76 E+01$ & $4.91 \mathrm{E}+01$ & $7.1 \%$ \\
\hline Th (IE) & $<4.20 \mathrm{E}+01$ & $<4.08 \mathrm{E}+01$ & $<1.94 E+02$ & $<1.79 E+02$ & $<4.14 \mathrm{E}+01$ & - \\
\hline $\mathrm{Ti}$ (IE) & $7.76 E+01$ & $9.53 E+01$ & $2.04 \mathrm{E}+02$ & $2.01 \mathrm{E}+02$ & $2.03 \mathrm{E}+02$ & $1.0 \%$ \\
\hline $\mathrm{U}$ (IE) & $6.41 \mathrm{E}+02$ & $6.94 \mathrm{E}+02$ & $<2.36 E+03$ & $<2.18 E+03$ & $6.68 \mathrm{E}+02$ & $5.6 \%$ \\
\hline V (IE) & $<3.69 \mathrm{E}+00$ & $<3.59 \mathrm{E}+00$ & $<3.39 E+01$ & $<3.14 E+01$ & $<3.64 \mathrm{E}+00$ & - \\
\hline Zn (IE) & $3.79 \mathrm{E}+02$ & $3.60 \mathrm{E}+02$ & $5.99 \mathrm{E}+02$ & $6.07 \mathrm{E}+02$ & $4.86 \mathrm{E}+02$ & $28 \%$ \\
\hline $\mathrm{Zr}$ (IE) & $5.27 \mathrm{E}+01$ & $6.38 \mathrm{E}+01$ & - & - & $5.83 \mathrm{E}+01$ & $13 \%$ \\
\hline $\mathrm{Hg}(\mathrm{CV})$ & $1.39 \mathrm{E}+03$ & $1.14 \mathrm{E}+03$ & - & - & $1.27 \mathrm{E}+03$ & $14 \%$ \\
\hline${ }^{232} \mathrm{Th}$ (IM) & $3.28 \mathrm{E}-01$ & $3.44 \mathrm{E}-01$ & $8.90 \mathrm{E}-01$ & 4.42E-01 & $5.01 \mathrm{E}-01$ & $53 \%$ \\
\hline${ }^{233} \mathrm{U}(\mathrm{IM})$ & $<1.96 \mathrm{E}-01$ & $<1.91 \mathrm{E}-01$ & $<1.81 \mathrm{E}+00$ & $<6.69 \mathrm{E}-01$ & $<7.15 \mathrm{E}-01$ & - \\
\hline${ }^{234} \mathrm{U}$ (IM) & $<1.96 \mathrm{E}-01$ & $<1.91 \mathrm{E}-01$ & $<1.81 \mathrm{E}+00$ & $<6.69 \mathrm{E}-01$ & $<7.15 \mathrm{E}-01$ & - \\
\hline${ }^{235} \mathrm{U}(\mathrm{IM})$ & $2.95 \mathrm{E}+00$ & $3.31 \mathrm{E}+00$ & $1.08 \mathrm{E}+01$ & $3.33 \mathrm{E}+00$ & $5.09 \mathrm{E}+00$ & $74 \%$ \\
\hline${ }^{236} \mathrm{U}(\mathrm{IM})$ & $4.02 \mathrm{E}-01$ & $4.06 \mathrm{E}-01$ & $<1.81 \mathrm{E}+00$ & $<6.69 \mathrm{E}-01$ & 4.04E-01 & $0.7 \%$ \\
\hline${ }^{238} \mathrm{U}(\mathrm{IM})$ & $7.76 \mathrm{E}+01$ & $8.43 \mathrm{E}+01$ & $2.95 \mathrm{E}+02$ & $7.48 \mathrm{E}+01$ & $1.33 \mathrm{E}+02$ & $81 \%$ \\
\hline U-total (IM) & $8.09 \mathrm{E}+01$ & $8.80 \mathrm{E}+01$ & $3.06 \mathrm{E}+02$ & $7.81 \mathrm{E}+01$ & $1.38 \mathrm{E}+02$ & $81 \%$ \\
\hline${ }^{90} \mathrm{Sr}(\mathrm{BS})$ & $2.88 \mathrm{E}+00$ & $3.03 \mathrm{E}+00$ & $7.33 \mathrm{E}+00$ & $2.95 \mathrm{E}+00$ & $4.05 \mathrm{E}+00$ & $54 \%$ \\
\hline${ }^{137} \mathrm{Cs}$ (GS) & $1.18 \mathrm{E}+01$ & $1.14 \mathrm{E}+01$ & $1.44 \mathrm{E}+01$ & $1.21 \mathrm{E}+01$ & $1.24 \mathrm{E}+01$ & $11 \%$ \\
\hline${ }^{238} \mathrm{Pu}$ (SA) & $9.81 \mathrm{E}-02$ & $1.11 \mathrm{E}-01$ & $1.91 \mathrm{E}-01$ & $6.66 \mathrm{E}-02$ & $1.17 \mathrm{E}-01$ & $45 \%$ \\
\hline${ }^{99}$ Tc (IM) & $7.20 \mathrm{E}+00$ & $7.87 \mathrm{E}+00$ & $1.91 \mathrm{E}+01$ & $7.51 \mathrm{E}+00$ & $1.04 \mathrm{E}+01$ & $56 \%$ \\
\hline${ }^{237} \mathrm{~Np}$ (IM) & $1.53 \mathrm{E}+00$ & $1.86 \mathrm{E}+00$ & $4.20 \mathrm{E}+00$ & $1.45 \mathrm{E}+00$ & $2.26 \mathrm{E}+00$ & $58 \%$ \\
\hline${ }^{239} \mathrm{Pu}$ (IM) & $3.08 \mathrm{E}+00$ & $3.65 \mathrm{E}+00$ & $<4.51 \mathrm{E}+00$ & $<1.67 \mathrm{E}+00$ & $3.37 \mathrm{E}+00$ & $12 \%$ \\
\hline${ }^{240} \mathrm{Pu}$ (IM) & $4.71 \mathrm{E}-01$ & $4.88 \mathrm{E}-01$ & $<1.81 \mathrm{E}+00$ & $<6.69 \mathrm{E}-01$ & $4.80 \mathrm{E}-01$ & $2.5 \%$ \\
\hline
\end{tabular}

Values in italics were not used in calculating the average. Divide $\mathrm{mg} / \mathrm{kg}$ values by $1 \mathrm{E}+04$ to convert to wt $\%$ basis. 
SRNL-STI-2012-00309

Revision 0

Table 3-10. Composition of the Oxalic Acid Leached Solids from Tank 16H Annulus Sample HTF-16-E-2 (continued)

\begin{tabular}{|c|c|c|c|c|c|c|}
\hline Analyte (Method) & $\begin{array}{c}\text { Aqua Regia } \\
\text { Replicate } 1 \\
\text { (dpm/g) } \\
\end{array}$ & $\begin{array}{c}\text { Aqua Regia } \\
\text { Replicate } 2 \\
\text { (dpm/g) }\end{array}$ & $\begin{array}{c}\text { Per Fusion } \\
\text { Replicate } 1 \\
\text { (dpm/g) }\end{array}$ & $\begin{array}{c}\text { Per Fusion } \\
\text { Replicate } 2 \\
\text { (dpm/g) }\end{array}$ & $\begin{array}{l}\text { Average } \\
(\mathrm{dpm} / \mathrm{g})\end{array}$ & $\%$ RSD \\
\hline${ }^{90} \mathrm{Sr}$ (BS) & $8.78 \mathrm{E}+08$ & $9.23 \mathrm{E}+08$ & $2.23 \mathrm{E}+09$ & $8.99 \mathrm{E}+08$ & $1.23 \mathrm{E}+09$ & $54 \%$ \\
\hline${ }^{137} \mathrm{Cs}$ (GS) & $2.27 \mathrm{E}+09$ & $2.19 \mathrm{E}+09$ & $2.78 \mathrm{E}+09$ & $2.33 \mathrm{E}+09$ & $2.39 \mathrm{E}+09$ & $11 \%$ \\
\hline${ }^{238} \mathrm{Pu}(\mathrm{SA})$ & $3.73 \mathrm{E}+06$ & $4.23 \mathrm{E}+06$ & $7.25 \mathrm{E}+06$ & $2.53 \mathrm{E}+06$ & $4.44 \mathrm{E}+06$ & $45 \%$ \\
\hline${ }^{239 / 240} \mathrm{Pu}(\mathrm{SA})$ & $5.95 \mathrm{E}+05$ & $7.58 \mathrm{E}+05$ & $1.24 \mathrm{E}+06$ & $4.43 \mathrm{E}+05$ & $7.59 \mathrm{E}+05$ & $46 \%$ \\
\hline${ }^{99} \mathrm{Tc}$ (IM) & $2.71 \mathrm{E}+05$ & $2.96 \mathrm{E}+05$ & $7.19 \mathrm{E}+05$ & $2.83 \mathrm{E}+05$ & $3.92 \mathrm{E}+05$ & $56 \%$ \\
\hline${ }^{232} \mathrm{Th}$ (IM) & $3.28 \mathrm{E}-01$ & $3.44 \mathrm{E}-01$ & $8.90 \mathrm{E}-01$ & 4.42E-01 & $5.01 \mathrm{E}-01$ & $53 \%$ \\
\hline${ }^{233} \mathrm{U}$ (IM) & $<4.20 \mathrm{E}+03$ & $<4.09 \mathrm{E}+03$ & $<3.87 \mathrm{E}+04$ & $<1.43 \mathrm{E}+04$ & $<1.53 \mathrm{E}+04$ & - \\
\hline${ }^{234} \mathrm{U}$ (IM) & $<2.72 \mathrm{E}+03$ & $<2.65 \mathrm{E}+03$ & $<2.51 \mathrm{E}+04$ & $<9.30 \mathrm{E}+03$ & $<9.94 \mathrm{E}+03$ & - \\
\hline${ }^{235} \mathrm{U}$ (IM) & $1.42 \mathrm{E}+01$ & $1.59 \mathrm{E}+01$ & $5.16 \mathrm{E}+01$ & $1.60 \mathrm{E}+01$ & $2.44 \mathrm{E}+01$ & $74 \%$ \\
\hline${ }^{236} \mathrm{U}$ (IM) & $5.77 \mathrm{E}+01$ & $5.83 \mathrm{E}+01$ & $<2.59 \mathrm{E}+02$ & $<9.60 \mathrm{E}+01$ & $5.80 \mathrm{E}+01$ & $0.7 \%$ \\
\hline${ }^{237} \mathrm{~Np}$ (IM) & $2.39 \mathrm{E}+03$ & $2.91 \mathrm{E}+03$ & $6.56 \mathrm{E}+03$ & $2.27 \mathrm{E}+03$ & $3.54 \mathrm{E}+03$ & $58 \%$ \\
\hline${ }^{238} \mathrm{U}$ (IM) & $5.79 \mathrm{E}+01$ & $6.29 \mathrm{E}+01$ & $2.20 \mathrm{E}+02$ & $5.58 \mathrm{E}+01$ & $9.92 \mathrm{E}+01$ & $81 \%$ \\
\hline${ }^{239} \mathrm{Pu}$ (IM) & $4.20 \mathrm{E}+05$ & $4.97 \mathrm{E}+05$ & $<6.14 \mathrm{E}+05$ & $<2.28 \mathrm{E}+05$ & $4.58 \mathrm{E}+05$ & $12 \%$ \\
\hline${ }^{240} \mathrm{Pu}$ (IM) & $2.36 \mathrm{E}+05$ & $2.44 \mathrm{E}+05$ & $<9.04 \mathrm{E}+05$ & $<3.35 \mathrm{E}+05$ & $2.40 \mathrm{E}+05$ & $2.5 \%$ \\
\hline
\end{tabular}

Values in italics were not used in calculating the average. Divide $\mathrm{mg} / \mathrm{kg}$ values by $1 \mathrm{E}+04$ to convert to wt $\%$ basis. 
Table 3-11. Composition of the Oxalic Acid Leached Solids from Tank 16H Annulus Sample HTF-16-N-1

\begin{tabular}{|c|c|c|c|c|c|c|}
\hline Analyte (Method) & $\begin{array}{c}\text { Aqua Regia } \\
\text { Replicate } 1 \\
\text { (mg/kg) }\end{array}$ & $\begin{array}{c}\text { Aqua Regia } \\
\text { Replicate } 2 \\
\text { (mg/kg) }\end{array}$ & $\begin{array}{c}\text { Per Fusion } \\
\text { Replicate } 1 \\
\text { (mg/kg) }\end{array}$ & $\begin{array}{c}\text { Per Fusion } \\
\text { Replicate } 2 \\
\text { (mg/kg) }\end{array}$ & $\begin{array}{c}\text { Average } \\
(\mathbf{m g} / \mathbf{k g})\end{array}$ & $\%$ RSD \\
\hline $\mathrm{Ag}$ (IE) & $<1.08 \mathrm{E}+02$ & $<1.06 \mathrm{E}+02$ & $<1.05 E+03$ & $<1.13 E+03$ & $<1.07 \mathrm{E}+02$ & - \\
\hline $\mathrm{Al}$ (IE) & $6.62 \mathrm{E}+04$ & $8.20 \mathrm{E}+04$ & $1.19 \mathrm{E}+05$ & $1.14 \mathrm{E}+05$ & $9.53 \mathrm{E}+04$ & $27 \%$ \\
\hline B (IE) & $<1.51 \mathrm{E}+01$ & $<1.49 \mathrm{E}+01$ & $<1.47 E+02$ & $<1.58 E+02$ & $<1.50 \mathrm{E}+01$ & - \\
\hline $\mathrm{Ba}$ (IE) & $3.71 \mathrm{E}+01$ & $4.09 \mathrm{E}+01$ & $1.41 E+02$ & $7.58 E+01$ & $3.90 \mathrm{E}+01$ & $6.9 \%$ \\
\hline $\mathrm{Be}$ (IE) & $<4.49 \mathrm{E}-01$ & $<4.41 \mathrm{E}-01$ & $<5.84 E+00$ & $<6.25 E+00$ & $<4.45 \mathrm{E}-01$ & - \\
\hline $\mathrm{Ca}$ (IE) & $3.07 \mathrm{E}+03$ & $4.23 \mathrm{E}+03$ & $6.66 E+03$ & $5.06 E+03$ & $3.65 \mathrm{E}+03$ & $22 \%$ \\
\hline $\mathrm{Cd}$ (IE) & $<2.32 \mathrm{E}+00$ & $<2.28 \mathrm{E}+00$ & $<2.26 E+01$ & $<2.42 E+01$ & $<2.30 \mathrm{E}+00$ & - \\
\hline $\mathrm{Ce}$ (IE) & $<5.30 \mathrm{E}+01$ & $<5.20 \mathrm{E}+01$ & $<5.16 E+02$ & $<5.52 E+02$ & $<5.25 \mathrm{E}+01$ & - \\
\hline Co (IE) & $<7.27 \mathrm{E}+00$ & $<7.13 \mathrm{E}+00$ & $<6.20 E+01$ & $<6.64 E+01$ & $<7.20 \mathrm{E}+00$ & - \\
\hline Cr (IE) & $8.85 \mathrm{E}+01$ & $1.51 \mathrm{E}+02$ & $8.76 \mathrm{E}+01$ & $<6.41 E+01$ & $1.09 \mathrm{E}+02$ & $33 \%$ \\
\hline $\mathrm{Cu}$ (IE) & $9.01 \mathrm{E}+01$ & $1.15 \mathrm{E}+02$ & $7.52 \mathrm{E}+01$ & $6.80 \mathrm{E}+01$ & $8.71 \mathrm{E}+01$ & $24 \%$ \\
\hline $\mathrm{Fe}(\mathrm{IE})$ & $1.09 \mathrm{E}+04$ & $1.15 \mathrm{E}+04$ & $2.50 \mathrm{E}+04$ & $4.84 \mathrm{E}+03$ & $1.31 \mathrm{E}+04$ & $65 \%$ \\
\hline Gd (IE) & $<1.61 \mathrm{E}+01$ & $<1.58 \mathrm{E}+01$ & $<1.57 E+02$ & $<1.68 E+02$ & $<1.60 \mathrm{E}+01$ & - \\
\hline $\mathrm{K}$ (IE) & $<1.92 \mathrm{E}+02$ & $2.48 \mathrm{E}+02$ & $3.89 E+03$ & $4.26 E+03$ & $2.20 \mathrm{E}+02$ & $18 \%$ \\
\hline La (IE) & $1.39 \mathrm{E}+01$ & $1.67 \mathrm{E}+01$ & $<4.89 E+01$ & $<5.23 E+01$ & $1.53 \mathrm{E}+01$ & $13 \%$ \\
\hline Li (IE) & $<1.09 \mathrm{E}+01$ & $<1.07 \mathrm{E}+01$ & $<1.07 E+02$ & $<1.14 E+02$ & $<1.08 \mathrm{E}+01$ & - \\
\hline $\mathrm{Mg}$ (IE) & $2.53 \mathrm{E}+02$ & $4.25 \mathrm{E}+02$ & $2.18 \mathrm{E}+02$ & $2.48 \mathrm{E}+02$ & $2.86 \mathrm{E}+02$ & $33 \%$ \\
\hline Mn (IE) & $6.79 \mathrm{E}+01$ & $9.29 \mathrm{E}+01$ & $1.23 \mathrm{E}+02$ & $4.77 \mathrm{E}+01$ & $8.29 \mathrm{E}+01$ & $39 \%$ \\
\hline Mo (IE) & $<4.62 \mathrm{E}+01$ & $<4.54 \mathrm{E}+01$ & $<1.96 E+02$ & $<2.09 E+02$ & $<4.58 \mathrm{E}+01$ & - \\
\hline $\mathrm{Na}$ (IE) & $4.84 \mathrm{E}+04$ & $5.41 \mathrm{E}+04$ & - & - & $5.13 \mathrm{E}+04$ & $7.9 \%$ \\
\hline $\mathrm{Ni}$ (IE) & $<3.41 \mathrm{E}+01$ & $<3.35 \mathrm{E}+01$ & $<3.32 E+02$ & $<3.55 E+02$ & $<3.38 \mathrm{E}+01$ & - \\
\hline $\mathrm{P}$ (IE) & $<6.49 \mathrm{E}+01$ & $<6.37 \mathrm{E}+01$ & $<1.20 E+03$ & $<1.28 E+03$ & $<6.43 \mathrm{E}+01$ & - \\
\hline $\mathrm{Pb}$ (IE) & $3.55 \mathrm{E}+02$ & $3.63 \mathrm{E}+02$ & $5.52 \mathrm{E}+02$ & $6.32 \mathrm{E}+02$ & $4.76 \mathrm{E}+02$ & $29 \%$ \\
\hline S (IE) & $<5.62 \mathrm{E}+02$ & $5.78 \mathrm{E}+02$ & $<5.47 E+03$ & $<5.86 E+03$ & $5.70 \mathrm{E}+02$ & $2.0 \%$ \\
\hline $\mathrm{Sb}$ (IE) & $<9.57 \mathrm{E}+01$ & $<9.39 \mathrm{E}+01$ & $<7.78 E+02$ & $<8.33 E+02$ & $<9.48 \mathrm{E}+01$ & - \\
\hline Si (IE) & - & - & $2.19 \mathrm{E}+05$ & $2.11 \mathrm{E}+05$ & $2.15 \mathrm{E}+05$ & $2.6 \%$ \\
\hline Sn (IE) & $<4.20 \mathrm{E}+01$ & $<4.13 \mathrm{E}+01$ & $<4.09 E+02$ & $<4.38 E+02$ & $<4.17 \mathrm{E}+01$ & - \\
\hline
\end{tabular}

Values in italics were not used in calculating the average. Divide $\mathrm{mg} / \mathrm{kg}$ values by $1 \mathrm{E}+04$ to convert to wt $\%$ basis. 
SRNL-STI-2012-00309

Revision 0

Table 3-11. Composition of the Oxalic Acid Leached Solids from Tank 16H Annulus Sample HTF-16-N-1 (continued)

\begin{tabular}{|c|c|c|c|c|c|c|}
\hline Analyte (Method) & $\begin{array}{c}\text { Aqua Regia } \\
\text { Replicate } 1 \\
\text { (mg/kg) }\end{array}$ & $\begin{array}{c}\text { Aqua Regia } \\
\text { Replicate } 2 \\
\text { (mg/kg) }\end{array}$ & $\begin{array}{c}\text { Per Fusion } \\
\text { Replicate } 1 \\
\text { (mg/kg) }\end{array}$ & $\begin{array}{c}\text { Per Fusion } \\
\text { Replicate } 2 \\
\text { (mg/kg) }\end{array}$ & $\begin{array}{c}\text { Average } \\
\text { (mg/kg) }\end{array}$ & $\%$ RSD \\
\hline $\mathrm{Sr}$ (IE) & $1.23 \mathrm{E}+01$ & $1.30 \mathrm{E}+01$ & $3.94 E+01$ & $3.75 E+01$ & $1.27 \mathrm{E}+01$ & $3.9 \%$ \\
\hline Th (IE) & $<4.01 \mathrm{E}+01$ & $<3.93 \mathrm{E}+01$ & $<1.96 E+02$ & $<2.09 E+02$ & $<3.97 \mathrm{E}+01$ & - \\
\hline $\mathrm{Ti}$ (IE) & $9.78 E+01$ & $1.53 E+02$ & $4.33 \mathrm{E}+02$ & $3.21 \mathrm{E}+02$ & $3.77 \mathrm{E}+02$ & $21 \%$ \\
\hline $\mathrm{U}$ (IE) & $<2.45 \mathrm{E}+02$ & $2.56 \mathrm{E}+02$ & $<2.38 E+03$ & $<2.55 E+03$ & $2.56 \mathrm{E}+02$ & - \\
\hline V (IE) & $6.44 \mathrm{E}+00$ & $8.82 \mathrm{E}+00$ & $<3.43 E+01$ & $<3.67 E+01$ & $7.63 \mathrm{E}+00$ & $22 \%$ \\
\hline Zn (IE) & $3.07 \mathrm{E}+02$ & $3.37 \mathrm{E}+02$ & $6.16 \mathrm{E}+02$ & $3.90 \mathrm{E}+02$ & $4.13 \mathrm{E}+02$ & $34 \%$ \\
\hline Zr (IE) & $2.80 \mathrm{E}+01$ & $3.51 \mathrm{E}+01$ & - & - & $3.16 \mathrm{E}+01$ & $16 \%$ \\
\hline $\mathrm{Hg}(\mathrm{CV})$ & $1.27 \mathrm{E}+03$ & $1.73 \mathrm{E}+03$ & - & - & $1.50 \mathrm{E}+03$ & $22 \%$ \\
\hline${ }^{232} \mathrm{Th}(\mathrm{IM})$ & $2.05 \mathrm{E}+00$ & $1.94 \mathrm{E}+00$ & $2.71 \mathrm{E}+00$ & $1.40 \mathrm{E}+00$ & $2.03 \mathrm{E}+00$ & $26 \%$ \\
\hline${ }^{233} \mathrm{U}(\mathrm{IM})$ & $<1.87 \mathrm{E}-01$ & $<1.84 \mathrm{E}-01$ & $<7.30 \mathrm{E}-01$ & $<7.81 \mathrm{E}-01$ & $<4.71 \mathrm{E}-01$ & - \\
\hline${ }^{234} \mathrm{U}(\mathrm{IM})$ & $<1.87 \mathrm{E}-01$ & $<1.84 \mathrm{E}-01$ & $<7.30 \mathrm{E}-01$ & $<7.81 \mathrm{E}-01$ & $<4.71 \mathrm{E}-01$ & - \\
\hline${ }^{235} \mathrm{U}(\mathrm{IM})$ & $1.04 \mathrm{E}+00$ & $1.01 \mathrm{E}+00$ & $1.53 \mathrm{E}+00$ & $1.91 \mathrm{E}+00$ & $1.37 \mathrm{E}+00$ & $31 \%$ \\
\hline${ }^{236} \mathrm{U}(\mathrm{IM})$ & $<1.87 \mathrm{E}-01$ & $<1.84 \mathrm{E}-01$ & $<7.30 \mathrm{E}-01$ & $<7.81 \mathrm{E}-01$ & $<4.71 \mathrm{E}-01$ & - \\
\hline${ }^{238} \mathrm{U}(\mathrm{IM})$ & $2.19 \mathrm{E}+01$ & $2.20 \mathrm{E}+01$ & $2.36 \mathrm{E}+01$ & $2.63 \mathrm{E}+01$ & $2.34 \mathrm{E}+01$ & $8.8 \%$ \\
\hline U-total (IM) & $2.29 \mathrm{E}+01$ & $2.30 \mathrm{E}+01$ & $2.51 \mathrm{E}+01$ & $2.82 \mathrm{E}+01$ & $2.48 \mathrm{E}+01$ & $10 \%$ \\
\hline${ }^{90} \mathrm{Sr}(\mathrm{BS})$ & $1.44 \mathrm{E}+00$ & $1.40 \mathrm{E}+00$ & $1.17 \mathrm{E}+00$ & 8.94E-01 & $1.23 \mathrm{E}+00$ & $21 \%$ \\
\hline${ }^{137} \mathrm{Cs}$ (GS) & $1.37 \mathrm{E}+01$ & $1.76 \mathrm{E}+01$ & $2.31 \mathrm{E}+01$ & $2.29 \mathrm{E}+01$ & $1.93 \mathrm{E}+01$ & $24 \%$ \\
\hline${ }^{238} \mathrm{Pu}(\mathrm{SA})$ & $3.37 \mathrm{E}-02$ & 4.13E-02 & $3.63 \mathrm{E}-02$ & $3.05 \mathrm{E}-02$ & $3.55 \mathrm{E}-02$ & $13 \%$ \\
\hline${ }^{99} \mathrm{Tc}(\mathrm{IM})$ & $7.96 \mathrm{E}+00$ & $1.17 \mathrm{E}+01$ & $7.34 \mathrm{E}+00$ & $6.18 \mathrm{E}+00$ & $8.28 \mathrm{E}+00$ & $29 \%$ \\
\hline${ }^{237} \mathrm{~Np}$ (IM) & $3.45 \mathrm{E}-01$ & 4.14E-01 & $<7.30 \mathrm{E}-01$ & $<7.81 \mathrm{E}-01$ & $3.80 \mathrm{E}-01$ & $13 \%$ \\
\hline${ }^{239} \mathrm{Pu}$ (IM) & $9.50 \mathrm{E}-01$ & $1.20 \mathrm{E}+00$ & $<1.82 \mathrm{E}+00$ & $<1.95 \mathrm{E}+00$ & $1.08 \mathrm{E}+00$ & $17 \%$ \\
\hline${ }^{240} \mathrm{Pu}$ (IM) & $<1.87 \mathrm{E}-01$ & $<1.84 \mathrm{E}-01$ & $<7.30 \mathrm{E}-01$ & $<7.81 \mathrm{E}-01$ & $<4.71 \mathrm{E}-01$ & - \\
\hline
\end{tabular}

Values in italics were not used in calculating the average. Divide $\mathrm{mg} / \mathrm{kg}$ values by $1 \mathrm{E}+04$ to convert to wt $\%$ basis. 
SRNL-STI-2012-00309

Revision 0

Table 3-11. Composition of the Oxalic Acid Leached Solids from Tank 16H Annulus Sample HTF-16-N-1 (continued)

\begin{tabular}{|c|c|c|c|c|c|c|}
\hline Analyte (Method) & $\begin{array}{c}\text { Aqua Regia } \\
\text { Replicate } 1 \\
\text { (dpm/g) }\end{array}$ & $\begin{array}{c}\text { Aqua Regia } \\
\text { Replicate } 2 \\
\text { (dpm/g) }\end{array}$ & $\begin{array}{c}\text { Per Fusion } \\
\text { Replicate } 1 \\
\text { (dpm/g) }\end{array}$ & $\begin{array}{c}\text { Per Fusion } \\
\text { Replicate } 2 \\
\text { (dpm/g) }\end{array}$ & $\begin{array}{l}\text { Average } \\
\text { (dpm/g) }\end{array}$ & $\%$ RSD \\
\hline${ }^{90} \mathrm{Sr}(\mathrm{BS})$ & $4.39 \mathrm{E}+08$ & $4.27 \mathrm{E}+08$ & $3.57 \mathrm{E}+08$ & $2.72 \mathrm{E}+08$ & $3.74 \mathrm{E}+08$ & $21 \%$ \\
\hline${ }^{137} \mathrm{Cs}$ (GS) & $2.63 \mathrm{E}+09$ & $3.39 \mathrm{E}+09$ & $4.44 \mathrm{E}+09$ & $4.41 \mathrm{E}+09$ & $3.72 \mathrm{E}+09$ & $24 \%$ \\
\hline${ }^{238} \mathrm{Pu}(\mathrm{SA})$ & $1.28 \mathrm{E}+06$ & $1.57 \mathrm{E}+06$ & $1.38 \mathrm{E}+06$ & $1.16 \mathrm{E}+06$ & $1.35 \mathrm{E}+06$ & $13 \%$ \\
\hline${ }^{239 / 240} \mathrm{Pu}(\mathrm{SA})$ & $1.76 \mathrm{E}+05$ & $2.35 \mathrm{E}+05$ & $2.05 \mathrm{E}+05$ & $1.77 \mathrm{E}+05$ & $1.98 \mathrm{E}+05$ & $14 \%$ \\
\hline${ }^{99} \mathrm{Tc}$ (IM) & $3.00 \mathrm{E}+05$ & $4.39 \mathrm{E}+05$ & $2.76 \mathrm{E}+05$ & $2.33 \mathrm{E}+05$ & $3.12 \mathrm{E}+05$ & $29 \%$ \\
\hline${ }^{232} \mathrm{Th}$ (IM) & $5.02 \mathrm{E}-01$ & 4.74E-01 & $6.62 \mathrm{E}-01$ & $3.43 \mathrm{E}-01$ & 4.95E-01 & $26 \%$ \\
\hline${ }^{233} \mathrm{U}$ (IM) & $<4.01 \mathrm{E}+03$ & $<3.94 \mathrm{E}+03$ & $<1.56 \mathrm{E}+04$ & $<1.67 \mathrm{E}+04$ & $<1.01 \mathrm{E}+04$ & - \\
\hline${ }^{234} \mathrm{U}$ (IM) & $<2.60 \mathrm{E}+03$ & $<2.55 \mathrm{E}+03$ & $<1.01 \mathrm{E}+04$ & $<1.09 \mathrm{E}+04$ & $<6.54 \mathrm{E}+03$ & - \\
\hline${ }^{235} \mathrm{U}$ (IM) & $4.97 \mathrm{E}+00$ & $4.86 \mathrm{E}+00$ & $7.36 \mathrm{E}+00$ & $9.15 \mathrm{E}+00$ & $6.58 \mathrm{E}+00$ & $31 \%$ \\
\hline${ }^{236} \mathrm{U}$ (IM) & $<2.69 \mathrm{E}+01$ & $<2.64 \mathrm{E}+01$ & $<1.05 \mathrm{E}+02$ & $<1.12 \mathrm{E}+02$ & $<6.76 \mathrm{E}+01$ & - \\
\hline${ }^{237} \mathrm{~Np}$ (IM) & $5.40 \mathrm{E}+02$ & $6.48 \mathrm{E}+02$ & $<1.14 \mathrm{E}+03$ & $<1.22 \mathrm{E}+03$ & $5.94 \mathrm{E}+02$ & $13 \%$ \\
\hline${ }^{238} \mathrm{U}$ (IM) & $1.63 \mathrm{E}+01$ & $1.64 \mathrm{E}+01$ & $1.76 \mathrm{E}+01$ & $1.96 \mathrm{E}+01$ & $1.75 \mathrm{E}+01$ & $8.8 \%$ \\
\hline${ }^{239} \mathrm{Pu}$ (IM) & $1.29 \mathrm{E}+05$ & $1.63 \mathrm{E}+05$ & $<2.48 \mathrm{E}+05$ & $<2.66 \mathrm{E}+05$ & $1.46 \mathrm{E}+05$ & $17 \%$ \\
\hline${ }^{240} \mathrm{Pu}$ (IM) & $<9.38 \mathrm{E}+04$ & $<9.21 \mathrm{E}+04$ & $<3.66 \mathrm{E}+05$ & $<3.91 \mathrm{E}+05$ & $<2.36 \mathrm{E}+05$ & - \\
\hline
\end{tabular}

Values in italics were not used in calculating the average. Divide $\mathrm{mg} / \mathrm{kg}$ values by $1 \mathrm{E}+04$ to convert to wt $\%$ basis. 
SRNL-STI-2012-00309

Revision 0

Table 3-12. Composition of the Oxalic Acid Leached Solids from Tank 16H Annulus Sample HTF-16-S-2

\begin{tabular}{|c|c|c|c|c|c|c|}
\hline Analyte (Method) & $\begin{array}{c}\text { Aqua Regia } \\
\text { Replicate } 1 \\
\text { (mg/kg) }\end{array}$ & $\begin{array}{c}\text { Aqua Regia } \\
\text { Replicate } 2 \\
\text { (mg/kg) }\end{array}$ & $\begin{array}{c}\text { Per Fusion } \\
\text { Replicate } 1 \\
\text { (mg/kg) }\end{array}$ & $\begin{array}{c}\text { Per Fusion } \\
\text { Replicate } 2 \\
\text { (mg/kg) }\end{array}$ & $\begin{array}{c}\text { Average } \\
(\mathbf{m g} / \mathbf{k g})\end{array}$ & $\%$ RSD \\
\hline $\mathrm{Ag}$ (IE) & $<9.93 \mathrm{E}+01$ & $<1.04 \mathrm{E}+02$ & $<1.04 E+03$ & $<1.06 E+03$ & $<1.02 \mathrm{E}+02$ & - \\
\hline $\mathrm{Al}$ (IE) & $4.03 \mathrm{E}+04$ & $7.18 \mathrm{E}+04$ & $4.41 \mathrm{E}+04$ & $5.15 \mathrm{E}+04$ & $5.19 \mathrm{E}+04$ & $27 \%$ \\
\hline B (IE) & $<1.39 \mathrm{E}+01$ & $<1.45 \mathrm{E}+01$ & $<1.46 E+02$ & $<1.49 E+02$ & $<1.42 \mathrm{E}+01$ & - \\
\hline $\mathrm{Ba}$ (IE) & $7.89 \mathrm{E}+01$ & $1.11 \mathrm{E}+02$ & $1.08 E+02$ & $1.76 E+02$ & $9.50 \mathrm{E}+01$ & $24 \%$ \\
\hline $\mathrm{Be}$ (IE) & $<4.14 \mathrm{E}-01$ & $<4.32 \mathrm{E}-01$ & $<5.80 E+00$ & $<5.90 E+00$ & $<4.23 \mathrm{E}-01$ & - \\
\hline $\mathrm{Ca}$ (IE) & $3.79 \mathrm{E}+03$ & $3.95 \mathrm{E}+03$ & $5.78 E+03$ & $8.18 E+03$ & $3.87 \mathrm{E}+03$ & $2.9 \%$ \\
\hline $\mathrm{Cd}$ (IE) & $4.69 \mathrm{E}+00$ & $3.60 \mathrm{E}+00$ & $<2.25 E+01$ & $<2.29 E+01$ & $4.15 \mathrm{E}+00$ & $19 \%$ \\
\hline $\mathrm{Ce}$ (IE) & $1.13 \mathrm{E}+02$ & $1.09 \mathrm{E}+02$ & $<5.12 E+02$ & $<5.22 E+02$ & $1.11 \mathrm{E}+02$ & $2.5 \%$ \\
\hline Co (IE) & $<6.69 \mathrm{E}+00$ & $<6.98 \mathrm{E}+00$ & $<6.16 E+01$ & $<6.27 E+01$ & $<6.84 \mathrm{E}+00$ & - \\
\hline Cr (IE) & $1.16 \mathrm{E}+02$ & $2.04 \mathrm{E}+02$ & $1.75 \mathrm{E}+02$ & $2.44 \mathrm{E}+02$ & $1.85 \mathrm{E}+02$ & $29 \%$ \\
\hline $\mathrm{Cu}$ (IE) & $1.27 \mathrm{E}+02$ & $1.77 \mathrm{E}+02$ & $1.02 \mathrm{E}+02$ & $1.05 \mathrm{E}+02$ & $1.28 \mathrm{E}+02$ & $27 \%$ \\
\hline $\mathrm{Fe}(\mathrm{IE})$ & $2.41 \mathrm{E}+04$ & $3.42 \mathrm{E}+04$ & $1.51 \mathrm{E}+04$ & $1.77 \mathrm{E}+04$ & $2.28 \mathrm{E}+04$ & $37 \%$ \\
\hline Gd (IE) & $<1.48 \mathrm{E}+01$ & $<1.55 \mathrm{E}+01$ & $<1.56 E+02$ & $<1.59 E+02$ & $<1.52 \mathrm{E}+01$ & - \\
\hline $\mathrm{K}$ (IE) & $2.30 \mathrm{E}+02$ & $2.21 \mathrm{E}+02$ & $4.23 E+03$ & $5.40 E+03$ & $2.26 \mathrm{E}+02$ & $2.8 \%$ \\
\hline La (IE) & $4.69 \mathrm{E}+01$ & $4.71 \mathrm{E}+01$ & $4.93 \mathrm{E}+01$ & $9.59 \mathrm{E}+01$ & $5.98 \mathrm{E}+01$ & $40 \%$ \\
\hline Li (IE) & $<1.01 \mathrm{E}+01$ & $<1.05 \mathrm{E}+01$ & $1.07 E+02$ & $<1.08 E+02$ & $<1.03 \mathrm{E}+01$ & - \\
\hline $\mathrm{Mg}$ (IE) & $1.42 \mathrm{E}+03$ & $1.51 \mathrm{E}+03$ & $4.23 \mathrm{E}+03$ & $8.00 \mathrm{E}+03$ & $3.79 \mathrm{E}+03$ & $82 \%$ \\
\hline Mn (IE) & $1.56 \mathrm{E}+02$ & $2.39 \mathrm{E}+02$ & $1.25 \mathrm{E}+02$ & $1.73 \mathrm{E}+02$ & $1.73 \mathrm{E}+02$ & $28 \%$ \\
\hline Mo (IE) & $<4.26 \mathrm{E}+01$ & $<4.44 \mathrm{E}+01$ & $<1.94 E+02$ & $<1.98 E+02$ & $<4.35 \mathrm{E}+01$ & - \\
\hline $\mathrm{Na}$ (IE) & $3.33 \mathrm{E}+04$ & $3.29 \mathrm{E}+04$ & - & - & $3.31 \mathrm{E}+04$ & $0.9 \%$ \\
\hline $\mathrm{Ni}$ (IE) & $5.79 \mathrm{E}+01$ & $8.17 \mathrm{E}+01$ & $<3.30 E+02$ & $<3.36 E+02$ & $6.98 \mathrm{E}+01$ & $24 \%$ \\
\hline $\mathrm{P}$ (IE) & $<5.97 \mathrm{E}+01$ & $6.69 \mathrm{E}+01$ & $<1.19 E+03$ & $<1.21 E+03$ & $6.69 \mathrm{E}+01$ & - \\
\hline $\mathrm{Pb}$ (IE) & $8.25 \mathrm{E}+02$ & $2.89 \mathrm{E}+03$ & $1.27 \mathrm{E}+03$ & $6.61 \mathrm{E}+03$ & $2.90 \mathrm{E}+03$ & $91 \%$ \\
\hline S (IE) & $1.10 \mathrm{E}+03$ & $3.70 \mathrm{E}+03$ & $<5.43 E+03$ & $<5.54 E+03$ & $2.40 \mathrm{E}+03$ & $77 \%$ \\
\hline $\mathrm{Sb}$ (IE) & $<8.81 \mathrm{E}+01$ & $<9.19 \mathrm{E}+01$ & $<7.72 E+02$ & $<7.87 E+02$ & $<9.00 \mathrm{E}+01$ & - \\
\hline Si (IE) & - & - & $1.97 \mathrm{E}+05$ & $2.07 \mathrm{E}+05$ & $2.02 \mathrm{E}+05$ & $3.5 \%$ \\
\hline Sn (IE) & $<3.87 \mathrm{E}+01$ & $<4.04 \mathrm{E}+01$ & $<4.07 E+02$ & $<4.14 E+02$ & $<3.96 \mathrm{E}+01$ & - \\
\hline
\end{tabular}

Values in italics were not used in calculating the average. Divide $\mathrm{mg} / \mathrm{kg}$ values by $1 \mathrm{E}+04$ to convert to wt $\%$ basis. 
SRNL-STI-2012-00309

Revision 0

Table 3-12. Composition of the Oxalic Acid Leached Solids from Tank 16H Annulus Sample HTF-16-S-2 (continued)

\begin{tabular}{|c|c|c|c|c|c|c|}
\hline Analyte (Method) & $\begin{array}{c}\text { Aqua Regia } \\
\text { Replicate } 1 \\
\text { (mg/kg) }\end{array}$ & $\begin{array}{c}\text { Aqua Regia } \\
\text { Replicate } 2 \\
\text { (mg/kg) }\end{array}$ & $\begin{array}{c}\text { Per Fusion } \\
\text { Replicate } 1 \\
\text { (mg/kg) }\end{array}$ & $\begin{array}{c}\text { Per Fusion } \\
\text { Replicate } 2 \\
\text { (mg/kg) }\end{array}$ & $\begin{array}{c}\text { Average } \\
(\mathbf{m g} / \mathbf{k g})\end{array}$ & $\%$ RSD \\
\hline $\operatorname{Sr}(\mathrm{IE})$ & $3.87 \mathrm{E}+01$ & $3.98 \mathrm{E}+01$ & $5.94 E+01$ & $6.79 E+01$ & $3.93 \mathrm{E}+01$ & $2.0 \%$ \\
\hline Th (IE) & $<3.69 \mathrm{E}+01$ & $<3.85 \mathrm{E}+01$ & $<1.94 E+02$ & $<1.98 E+02$ & $<3.77 \mathrm{E}+01$ & - \\
\hline Ti (IE) & $6.37 E+01$ & $7.47 E+01$ & $1.75 \mathrm{E}+02$ & $3.16 \mathrm{E}+02$ & $2.46 \mathrm{E}+02$ & $41 \%$ \\
\hline $\mathrm{U}$ (IE) & $<2.25 \mathrm{E}+02$ & $<2.35 \mathrm{E}+02$ & $<2.37 E+03$ & $<2.41 E+03$ & $<2.30 \mathrm{E}+02$ & - \\
\hline V (IE) & $7.17 \mathrm{E}+00$ & $8.27 \mathrm{E}+00$ & $<3.41 E+01$ & $<3.47 E+01$ & $7.72 \mathrm{E}+00$ & $10 \%$ \\
\hline Zn (IE) & $2.77 \mathrm{E}+02$ & $4.59 \mathrm{E}+02$ & $3.94 \mathrm{E}+02$ & $4.47 \mathrm{E}+02$ & $3.94 \mathrm{E}+02$ & $21 \%$ \\
\hline Zr (IE) & $1.24 \mathrm{E}+02$ & $1.29 \mathrm{E}+02$ & - & - & $1.27 \mathrm{E}+02$ & $2.8 \%$ \\
\hline $\mathrm{Hg}(\mathrm{CV})$ & $2.41 \mathrm{E}+03$ & $5.03 \mathrm{E}+03$ & - & - & $3.72 \mathrm{E}+03$ & $50 \%$ \\
\hline${ }^{232} \mathrm{Th}$ (IM) & $1.34 \mathrm{E}+00$ & $1.92 \mathrm{E}+00$ & $3.24 \mathrm{E}+00$ & $3.24 \mathrm{E}+00$ & $2.43 \mathrm{E}+00$ & $39 \%$ \\
\hline${ }^{233} \mathrm{U}(\mathrm{IM})$ & $<1.72 \mathrm{E}-01$ & $<1.80 \mathrm{E}-01$ & $<7.25 \mathrm{E}-01$ & $<1.85 \mathrm{E}+00$ & $<7.30 \mathrm{E}-01$ & - \\
\hline${ }^{234} \mathrm{U}$ (IM) & $<1.72 \mathrm{E}-01$ & $<1.80 \mathrm{E}-01$ & $<7.25 \mathrm{E}-01$ & $<1.85 \mathrm{E}+00$ & $<7.30 \mathrm{E}-01$ & - \\
\hline${ }^{235} \mathrm{U}$ (IM) & $1.44 \mathrm{E}+00$ & $1.85 \mathrm{E}+00$ & $4.67 \mathrm{E}+00$ & $3.93 \mathrm{E}+00$ & $2.97 \mathrm{E}+00$ & $53 \%$ \\
\hline${ }^{236} \mathrm{U}(\mathrm{IM})$ & $<1.72 \mathrm{E}-01$ & $2.59 \mathrm{E}-01$ & $<7.25 \mathrm{E}-01$ & $<1.85 \mathrm{E}+00$ & $<7.50 \mathrm{E}-01$ & - \\
\hline${ }^{238} \mathrm{U}(\mathrm{IM})$ & $4.01 \mathrm{E}+01$ & $4.92 \mathrm{E}+01$ & $9.08 \mathrm{E}+01$ & $1.02 \mathrm{E}+02$ & $7.05 \mathrm{E}+01$ & $43 \%$ \\
\hline U-total (IM) & $4.15 \mathrm{E}+01$ & $5.13 \mathrm{E}+01$ & $9.54 \mathrm{E}+01$ & $1.06 \mathrm{E}+02$ & $7.35 \mathrm{E}+01$ & $43 \%$ \\
\hline${ }^{90} \mathrm{Sr}$ (BS) & $5.55 \mathrm{E}+00$ & $5.22 \mathrm{E}+00$ & $5.42 \mathrm{E}+00$ & $4.99 \mathrm{E}+00$ & $5.30 \mathrm{E}+00$ & $4.6 \%$ \\
\hline${ }^{137} \mathrm{Cs}$ (GS) & $1.12 \mathrm{E}+01$ & $1.22 \mathrm{E}+01$ & $1.14 \mathrm{E}+01$ & $8.98 \mathrm{E}+00$ & $1.09 \mathrm{E}+01$ & $12 \%$ \\
\hline${ }^{238} \mathrm{Pu}(\mathrm{SA})$ & 7.47E-02 & $8.79 \mathrm{E}-02$ & 7.81E-02 & $8.39 \mathrm{E}-02$ & $8.12 \mathrm{E}-02$ & $7.2 \%$ \\
\hline${ }^{99} \mathrm{Tc}$ (IM) & $1.65 \mathrm{E}+01$ & $1.79 \mathrm{E}+01$ & $2.53 \mathrm{E}+01$ & $2.09 \mathrm{E}+01$ & $2.01 \mathrm{E}+01$ & $19 \%$ \\
\hline${ }^{237} \mathrm{~Np}$ (IM) & $8.38 \mathrm{E}-01$ & $1.04 \mathrm{E}+00$ & $1.48 \mathrm{E}+00$ & $<1.85 \mathrm{E}+00$ & $1.30 \mathrm{E}+00$ & $35 \%$ \\
\hline${ }^{239} \mathrm{Pu}$ (IM) & $2.30 \mathrm{E}+00$ & $2.47 \mathrm{E}+00$ & $<1.81 \mathrm{E}+00$ & $<4.61 \mathrm{E}+00$ & $2.39 \mathrm{E}+00$ & $5.0 \%$ \\
\hline${ }^{240} \mathrm{Pu} \quad(\mathrm{IM})$ & $3.29 \mathrm{E}-01$ & $3.40 \mathrm{E}-01$ & $<7.25 \mathrm{E}-01$ & $<1.85 \mathrm{E}+00$ & $3.35 \mathrm{E}-01$ & $2.3 \%$ \\
\hline
\end{tabular}

Values in italics were not used in calculating the average. Divide $\mathrm{mg} / \mathrm{kg}$ values by $1 \mathrm{E}+04$ to convert to wt $\%$ basis. 
SRNL-STI-2012-00309

Revision 0

Table 3-12. Composition of the Oxalic Acid Leached Solids from Tank 16H Annulus Sample HTF-16-S-2 (continued)

\begin{tabular}{|c|c|c|c|c|c|c|}
\hline Analyte (Method) & $\begin{array}{c}\text { Aqua Regia } \\
\text { Replicate } 1 \\
\text { (dpm/g) }\end{array}$ & $\begin{array}{c}\text { Aqua Regia } \\
\text { Replicate } 2 \\
\text { (dpm/g) }\end{array}$ & $\begin{array}{c}\text { Per Fusion } \\
\text { Replicate } 1 \\
\text { (dpm/g) }\end{array}$ & $\begin{array}{c}\text { Per Fusion } \\
\text { Replicate } 2 \\
\text { (dpm/g) }\end{array}$ & $\begin{array}{l}\text { Average } \\
(\mathrm{dpm} / \mathrm{g})\end{array}$ & $\%$ RSD \\
\hline${ }^{90} \mathrm{Sr}(\mathrm{BS})$ & $1.69 \mathrm{E}+09$ & $1.59 \mathrm{E}+09$ & $1.65 \mathrm{E}+09$ & $1.52 \mathrm{E}+09$ & $1.61 \mathrm{E}+09$ & $4.6 \%$ \\
\hline${ }^{137} \mathrm{Cs}$ (GS) & $2.16 \mathrm{E}+09$ & $2.34 \mathrm{E}+09$ & $2.20 \mathrm{E}+09$ & $1.73 \mathrm{E}+09$ & $2.11 \mathrm{E}+09$ & $12 \%$ \\
\hline${ }^{238} \mathrm{Pu}(\mathrm{SA})$ & $2.84 \mathrm{E}+06$ & $3.34 \mathrm{E}+06$ & $2.97 \mathrm{E}+06$ & $3.19 \mathrm{E}+06$ & $3.09 \mathrm{E}+06$ & $7.2 \%$ \\
\hline${ }^{239 / 240} \mathrm{Pu}(\mathrm{SA})$ & $4.84 \mathrm{E}+05$ & $4.97 \mathrm{E}+05$ & $5.12 \mathrm{E}+05$ & $5.61 \mathrm{E}+05$ & $5.14 \mathrm{E}+05$ & $6.6 \%$ \\
\hline${ }^{99} \mathrm{Tc}$ (IM) & $6.20 \mathrm{E}+05$ & $6.75 \mathrm{E}+05$ & $9.54 \mathrm{E}+05$ & $7.85 \mathrm{E}+05$ & $7.59 \mathrm{E}+05$ & $19 \%$ \\
\hline${ }^{232} \mathrm{Th}$ (IM) & $3.28 \mathrm{E}-01$ & 4.68E-01 & 7.91E-01 & 7.91E-01 & $5.94 \mathrm{E}-01$ & $39 \%$ \\
\hline${ }^{233} \mathrm{U}$ (IM) & $<3.69 \mathrm{E}+03$ & $<3.85 \mathrm{E}+03$ & $<1.55 \mathrm{E}+04$ & $<3.95 \mathrm{E}+04$ & $<1.56 \mathrm{E}+04$ & - \\
\hline${ }^{234} \mathrm{U}$ (IM) & $<2.40 \mathrm{E}+03$ & $<2.50 \mathrm{E}+03$ & $<1.01 \mathrm{E}+04$ & $<2.56 \mathrm{E}+04$ & $<1.02 \mathrm{E}+04$ & - \\
\hline${ }^{235} \mathrm{U}$ (IM) & $6.91 \mathrm{E}+00$ & $8.87 \mathrm{E}+00$ & $2.24 \mathrm{E}+01$ & $1.89 \mathrm{E}+01$ & $1.43 \mathrm{E}+01$ & $53 \%$ \\
\hline${ }^{236} \mathrm{U}$ (IM) & $<2.48 \mathrm{E}+01$ & $3.72 \mathrm{E}+01$ & $<1.04 \mathrm{E}+02$ & $<2.65 \mathrm{E}+02$ & $<1.08 \mathrm{E}+02$ & - \\
\hline${ }^{237} \mathrm{~Np}$ (IM) & $1.31 \mathrm{E}+03$ & $1.62 \mathrm{E}+03$ & $2.32 \mathrm{E}+03$ & $<2.89 \mathrm{E}+03$ & $1.75 \mathrm{E}+03$ & $29 \%$ \\
\hline${ }^{238} \mathrm{U}$ (IM) & $2.99 \mathrm{E}+01$ & $3.67 \mathrm{E}+01$ & $6.77 \mathrm{E}+01$ & $7.59 \mathrm{E}+01$ & $5.26 \mathrm{E}+01$ & $43 \%$ \\
\hline${ }^{239} \mathrm{Pu}$ (IM) & $3.13 \mathrm{E}+05$ & $3.36 \mathrm{E}+05$ & $<2.47 \mathrm{E}+05$ & $<6.28 \mathrm{E}+05$ & $3.25 \mathrm{E}+05$ & $5.0 \%$ \\
\hline${ }^{240} \mathrm{Pu}$ (IM) & $1.65 \mathrm{E}+05$ & $1.70 \mathrm{E}+05$ & $<3.63 \mathrm{E}+05$ & $<9.24 \mathrm{E}+05$ & $1.68 \mathrm{E}+05$ & $2.3 \%$ \\
\hline
\end{tabular}

Values in italics were not used in calculating the average. Divide $\mathrm{mg} / \mathrm{kg}$ values by $1 \mathrm{E}+04$ to convert to wt $\%$ basis. 
SRNL-STI-2012-00309

Revision 0

Table 3-13. Composition of the Oxalic Acid Leached Solids from Tank 16H Annulus Sample HTF-16-W-1

\begin{tabular}{|c|c|c|c|c|c|c|}
\hline Analyte (Method) & $\begin{array}{c}\text { Aqua Regia } \\
\text { Replicate } 1 \\
\text { (mg/kg) }\end{array}$ & $\begin{array}{c}\text { Aqua Regia } \\
\text { Replicate } 2 \\
\text { (mg/kg) }\end{array}$ & $\begin{array}{c}\text { Per Fusion } \\
\text { Replicate } 1 \\
\text { (mg/kg) }\end{array}$ & $\begin{array}{c}\text { Per Fusion } \\
\text { Replicate } 2 \\
\text { (mg/kg) }\end{array}$ & $\begin{array}{c}\text { Average } \\
(\mathbf{m g} / \mathbf{k g})\end{array}$ & $\%$ RSD \\
\hline $\mathrm{Ag}$ (IE) & $<1.10 \mathrm{E}+02$ & $<1.10 \mathrm{E}+02$ & $<1.05 E+03$ & $<1.04 E+03$ & $<1.10 \mathrm{E}+02$ & - \\
\hline $\mathrm{Al}$ (IE) & $1.06 \mathrm{E}+05$ & $9.39 \mathrm{E}+04$ & $9.49 \mathrm{E}+04$ & $8.95 \mathrm{E}+04$ & $9.61 \mathrm{E}+04$ & $7.3 \%$ \\
\hline B (IE) & $<1.55 \mathrm{E}+01$ & $<1.54 \mathrm{E}+01$ & $<1.48 E+02$ & $<1.46 E+02$ & $<1.55 \mathrm{E}+01$ & - \\
\hline $\mathrm{Ba}$ (IE) & $1.20 \mathrm{E}+02$ & $1.05 \mathrm{E}+02$ & $1.61 E+02$ & $1.14 E+02$ & $1.13 \mathrm{E}+02$ & $9.4 \%$ \\
\hline $\mathrm{Be}$ (IE) & $<4.60 \mathrm{E}-01$ & $<4.56 \mathrm{E}-01$ & $<5.86 E+00$ & $<5.78 E+00$ & $<4.58 \mathrm{E}-01$ & - \\
\hline $\mathrm{Ca}$ (IE) & $4.80 \mathrm{E}+02$ & $1.39 \mathrm{E}+03$ & $3.33 E+03$ & $3.68 E+03$ & $9.35 \mathrm{E}+02$ & $69 \%$ \\
\hline $\mathrm{Cd}$ (IE) & $2.38 \mathrm{E}+00$ & $2.81 \mathrm{E}+00$ & $<2.27 \mathrm{E}+01$ & $<2.24 \mathrm{E}+01$ & $2.60 \mathrm{E}+00$ & $12 \%$ \\
\hline $\mathrm{Ce}$ (IE) & $2.03 \mathrm{E}+02$ & $1.66 \mathrm{E}+02$ & $<5.18 E+02$ & $<5.10 E+02$ & $1.85 \mathrm{E}+02$ & $14 \%$ \\
\hline Co (IE) & $<7.43 \mathrm{E}+00$ & $<7.38 \mathrm{E}+00$ & $<6.23 E+01$ & $<6.14 E+01$ & $<7.41 \mathrm{E}+00$ & - \\
\hline Cr (IE) & $8.17 \mathrm{E}+01$ & $7.95 \mathrm{E}+01$ & $1.59 \mathrm{E}+02$ & $1.74 \mathrm{E}+02$ & $1.24 \mathrm{E}+02$ & $40 \%$ \\
\hline $\mathrm{Cu}$ (IE) & $6.67 \mathrm{E}+01$ & $7.41 \mathrm{E}+01$ & $6.89 \mathrm{E}+01$ & $4.77 \mathrm{E}+01$ & $6.44 \mathrm{E}+01$ & $18 \%$ \\
\hline $\mathrm{Fe}(\mathrm{IE})$ & $1.07 \mathrm{E}+04$ & $1.19 \mathrm{E}+04$ & $2.65 \mathrm{E}+04$ & $2.00 \mathrm{E}+04$ & $1.73 \mathrm{E}+04$ & $43 \%$ \\
\hline Gd (IE) & $<1.65 \mathrm{E}+01$ & $<1.63 \mathrm{E}+01$ & $<1.58 E+02$ & $<1.55 E+02$ & $<1.64 \mathrm{E}+01$ & - \\
\hline $\mathrm{K}$ (IE) & $5.08 \mathrm{E}+02$ & $4.22 \mathrm{E}+02$ & $4.22 E+03$ & $4.02 E+03$ & $4.65 \mathrm{E}+02$ & $13 \%$ \\
\hline La (IE) & $7.46 \mathrm{E}+01$ & $6.75 \mathrm{E}+01$ & $9.38 \mathrm{E}+01$ & $6.71 \mathrm{E}+01$ & $7.58 \mathrm{E}+01$ & $17 \%$ \\
\hline Li (IE) & $<1.12 \mathrm{E}+01$ & $<1.11 \mathrm{E}+01$ & $<1.07 E+02$ & $<1.05 E+02$ & $<1.12 \mathrm{E}+01$ & - \\
\hline $\mathrm{Mg}$ (IE) & $1.41 \mathrm{E}+02$ & $1.39 \mathrm{E}+02$ & $1.88 \mathrm{E}+02$ & $1.33 \mathrm{E}+02$ & $1.50 \mathrm{E}+02$ & $17 \%$ \\
\hline Mn (IE) & $1.27 \mathrm{E}+02$ & $1.32 \mathrm{E}+02$ & $2.29 \mathrm{E}+02$ & $1.86 \mathrm{E}+02$ & $1.69 \mathrm{E}+02$ & $29 \%$ \\
\hline Mo (IE) & $<4.73 \mathrm{E}+01$ & $<4.69 \mathrm{E}+01$ & $<1.96 E+02$ & $<1.94 E+02$ & $<4.71 \mathrm{E}+01$ & - \\
\hline $\mathrm{Na}$ (IE) & $9.68 \mathrm{E}+04$ & $8.81 \mathrm{E}+04$ & - & - & $9.25 \mathrm{E}+04$ & $6.7 \%$ \\
\hline $\mathrm{Ni}$ (IE) & $9.95 \mathrm{E}+01$ & $1.32 \mathrm{E}+02$ & $<3.33 E+02$ & $<3.29 E+02$ & $1.16 \mathrm{E}+02$ & $20 \%$ \\
\hline $\mathrm{P}$ (IE) & $<6.64 \mathrm{E}+01$ & $<6.59 \mathrm{E}+01$ & $<1.20 E+03$ & $<1.18 E+03$ & $<6.62 \mathrm{E}+01$ & - \\
\hline $\mathrm{Pb}$ (IE) & $5.66 \mathrm{E}+02$ & $5.18 \mathrm{E}+02$ & $9.66 \mathrm{E}+02$ & $8.93 \mathrm{E}+02$ & $7.36 \mathrm{E}+02$ & $31 \%$ \\
\hline S (IE) & $8.46 \mathrm{E}+02$ & $<5.70 \mathrm{E}+02$ & $<5.49 E+03$ & $<5.42 E+03$ & $8.46 \mathrm{E}+02$ & - \\
\hline $\mathrm{Sb}$ (IE) & $<9.79 \mathrm{E}+01$ & $<9.71 \mathrm{E}+01$ & $<7.81 E+02$ & $<7.70 E+02$ & $<9.75 \mathrm{E}+01$ & - \\
\hline Si (IE) & - & - & $2.39 \mathrm{E}+05$ & $2.84 \mathrm{E}+05$ & $2.62 \mathrm{E}+05$ & $12 \%$ \\
\hline Sn (IE) & $<4.30 \mathrm{E}+01$ & $<4.27 \mathrm{E}+01$ & $<4.11 E+02$ & $<4.05 E+02$ & $<4.29 \mathrm{E}+01$ & - \\
\hline
\end{tabular}

Values in italics were not used in calculating the average. Divide $\mathrm{mg} / \mathrm{kg}$ values by $1 \mathrm{E}+04$ to convert to wt $\%$ basis. 
SRNL-STI-2012-00309

Revision 0

Table 3-13. Composition of the Oxalic Acid Leached Solids from Tank 16H Annulus Sample HTF-16-W-1 (continued)

\begin{tabular}{|l|c|c|c|c|c|c||}
\hline Analyte (Method) & $\begin{array}{c}\text { Aqua Regia } \\
\text { Replicate 1 } \\
\text { (mg/kg) }\end{array}$ & $\begin{array}{c}\text { Aqua Regia } \\
\text { Replicate 2 } \\
\text { (mg/kg) }\end{array}$ & $\begin{array}{c}\text { Per Fusion } \\
\text { Replicate 1 } \\
\text { (mg/kg) }\end{array}$ & $\begin{array}{c}\text { Per Fusion } \\
\text { Replicate 2 } \\
\text { (mg/kg) }\end{array}$ & $\begin{array}{c}\text { Average } \\
\text { (mg/kg) }\end{array}$ & \%RSD \\
\hline Sr (IE) & $2.87 \mathrm{E}+01$ & $2.83 \mathrm{E}+01$ & $5.86 E+01$ & $5.27 E+01$ & $2.85 \mathrm{E}+01$ & $1.0 \%$ \\
\hline Th (IE) & $<4.10 \mathrm{E}+01$ & $<4.07 \mathrm{E}+01$ & $<1.96 E+02$ & $<1.94 E+02$ & $<4.09 \mathrm{E}+01$ & - \\
\hline Ti (IE) & $3.72 E+01$ & $3.33 E+01$ & $1.07 \mathrm{E}+02$ & $7.36 \mathrm{E}+01$ & $9.03 \mathrm{E}+01$ & $26 \%$ \\
\hline $\mathrm{U}(\mathrm{IE})$ & $<2.50 \mathrm{E}+02$ & $<2.48 \mathrm{E}+02$ & $<2.39 E+03$ & $<2.36 E+03$ & $<2.49 \mathrm{E}+02$ & - \\
\hline $\mathrm{V}(\mathrm{IE})$ & $<3.60 \mathrm{E}+00$ & $<3.57 \mathrm{E}+00$ & $<3.44 E+01$ & $<3.39 E+01$ & $<3.59 \mathrm{E}+00$ & - \\
\hline $\mathrm{Zn}(\mathrm{IE})$ & $2.30 \mathrm{E}+02$ & $3.03 \mathrm{E}+02$ & $3.27 \mathrm{E}+02$ & $2.73 \mathrm{E}+02$ & $2.83 \mathrm{E}+02$ & $15 \%$ \\
\hline $\mathrm{Zr}(\mathrm{IE})$ & $2.47 \mathrm{E}+02$ & $2.06 \mathrm{E}+02$ & - & - & $2.27 \mathrm{E}+02$ & $13 \%$ \\
\hline $\mathrm{Hg}(\mathrm{CV})$ & $1.89 \mathrm{E}+03$ & $2.00 \mathrm{E}+03$ & - & - & $1.95 \mathrm{E}+03$ & $4.0 \%$ \\
\hline${ }^{232} \mathrm{Th}(\mathrm{IM})$ & $6.66 \mathrm{E}-01$ & $7.22 \mathrm{E}-01$ & $2.00 \mathrm{E}+00$ & $1.10 \mathrm{E}+00$ & $1.12 \mathrm{E}+00$ & $55 \%$ \\
\hline${ }^{233} \mathrm{U}(\mathrm{IM})$ & $<1.92 \mathrm{E}-01$ & $<1.90 \mathrm{E}-01$ & $<7.33 \mathrm{E}-01$ & $<7.22 \mathrm{E}-01$ & $<4.59 \mathrm{E}-01$ & - \\
\hline${ }^{234} \mathrm{U}(\mathrm{IM})$ & $<1.92 \mathrm{E}-01$ & $<1.90 \mathrm{E}-01$ & $<7.33 \mathrm{E}-01$ & $<7.22 \mathrm{E}-01$ & $<4.59 \mathrm{E}-01$ & - \\
\hline${ }^{235} \mathrm{U}(\mathrm{IM})$ & $3.99 \mathrm{E}+00$ & $3.32 \mathrm{E}+00$ & $4.91 \mathrm{E}+00$ & $3.29 \mathrm{E}+00$ & $3.88 \mathrm{E}+00$ & $20 \%$ \\
\hline${ }^{236} \mathrm{U}(\mathrm{IM})$ & $3.23 \mathrm{E}-01$ & $3.94 \mathrm{E}-01$ & $<7.33 \mathrm{E}-01$ & $<7.22 \mathrm{E}-01$ & $3.59 \mathrm{E}-01$ & $14 \%$ \\
\hline${ }^{238} \mathrm{U}(\mathrm{IM})$ & $1.02 \mathrm{E}+02$ & $8.68 \mathrm{E}+01$ & $1.09 \mathrm{E}+02$ & $7.19 \mathrm{E}+01$ & $9.24 \mathrm{E}+01$ & $18 \%$ \\
\hline $\mathrm{U}-\mathrm{total}(\mathrm{IM})$ & $1.07 \mathrm{E}+02$ & $9.05 \mathrm{E}+01$ & $1.14 \mathrm{E}+02$ & $7.52 \mathrm{E}+01$ & $9.65 \mathrm{E}+01$ & $18 \%$ \\
\hline${ }^{90} \mathrm{Sr}(\mathrm{BS})$ & $5.45 \mathrm{E}+00$ & $5.16 \mathrm{E}+00$ & $5.68 \mathrm{E}+00$ & $4.57 \mathrm{E}+00$ & $5.22 \mathrm{E}+00$ & $9.3 \%$ \\
\hline${ }^{137} \mathrm{Cs}(\mathrm{GS})$ & $2.09 \mathrm{E}+01$ & $1.92 \mathrm{E}+01$ & $2.17 \mathrm{E}+01$ & $1.82 \mathrm{E}+01$ & $2.00 \mathrm{E}+01$ & $7.9 \%$ \\
\hline${ }^{238} \mathrm{Pu}(\mathrm{SA})$ & $1.53 \mathrm{E}-01$ & $1.19 \mathrm{E}-01$ & $1.52 \mathrm{E}-01$ & $9.34 \mathrm{E}-02$ & $1.29 \mathrm{E}-01$ & $22 \%$ \\
\hline${ }^{99} \mathrm{Tc}(\mathrm{IM})$ & $1.74 \mathrm{E}+01$ & $1.96 \mathrm{E}+01$ & $3.36 \mathrm{E}+01$ & $2.42 \mathrm{E}+01$ & $2.37 \mathrm{E}+01$ & $30 \%$ \\
\hline${ }^{237} \mathrm{~Np}(\mathrm{IM})$ & $1.37 \mathrm{E}+00$ & $1.17 \mathrm{E}+00$ & $1.91 \mathrm{E}+00$ & $1.24 \mathrm{E}+00$ & $1.42 \mathrm{E}+00$ & $24 \%$ \\
\hline${ }^{239} \mathrm{Pu}(\mathrm{IM})$ & $4.66 \mathrm{E}+00$ & $3.94 \mathrm{E}+00$ & $<1.83 \mathrm{E}+00$ & $<1.81 \mathrm{E}+00$ & $4.30 \mathrm{E}+00$ & $12 \%$ \\
\hline$\left.{ }^{240} \mathrm{Pu}\right)$ & $6.12 \mathrm{E}-01$ & $5.05 \mathrm{E}-01$ & $8.13 \mathrm{E}-01$ & $<7.22 \mathrm{E}-01$ & $6.43 \mathrm{E}-01$ & $24 \%$ \\
\hline
\end{tabular}

Values in italics were not used in calculating the average. Divide $\mathrm{mg} / \mathrm{kg}$ values by $1 \mathrm{E}+04$ to convert to wt $\%$ basis. 
SRNL-STI-2012-00309

Revision 0

Table 3-13. Composition of the Oxalic Acid Leached Solids from Tank 16H Annulus Sample HTF-16-W-1 (continued)

\begin{tabular}{|c|c|c|c|c|c|c|}
\hline Analyte (Method) & $\begin{array}{c}\text { Aqua Regia } \\
\text { Replicate } 1 \\
\text { (dpm/g) } \\
\end{array}$ & $\begin{array}{c}\text { Aqua Regia } \\
\text { Replicate } 2 \\
\text { (dpm/g) }\end{array}$ & $\begin{array}{c}\text { Per Fusion } \\
\text { Replicate } 1 \\
\text { (dpm/g) }\end{array}$ & $\begin{array}{c}\text { Per Fusion } \\
\text { Replicate } 2 \\
\text { (dpm/g) }\end{array}$ & $\begin{array}{l}\text { Average } \\
\text { (dpm/g) }\end{array}$ & $\%$ RSD \\
\hline${ }^{90} \mathrm{Sr}$ (BS) & $1.66 \mathrm{E}+09$ & $1.57 \mathrm{E}+09$ & $1.73 \mathrm{E}+09$ & $1.39 \mathrm{E}+09$ & $1.59 \mathrm{E}+09$ & $9.3 \%$ \\
\hline${ }^{137} \mathrm{Cs}$ (GS) & $4.02 \mathrm{E}+09$ & $3.69 \mathrm{E}+09$ & $4.18 \mathrm{E}+09$ & $3.51 \mathrm{E}+09$ & $3.85 \mathrm{E}+09$ & $7.9 \%$ \\
\hline${ }^{238} \mathrm{Pu}(\mathrm{SA})$ & $5.80 \mathrm{E}+06$ & $4.53 \mathrm{E}+06$ & $5.78 \mathrm{E}+06$ & $3.55 \mathrm{E}+06$ & $4.92 \mathrm{E}+06$ & $22 \%$ \\
\hline${ }^{239 / 240} \mathrm{Pu}(\mathrm{SA})$ & $1.11 \mathrm{E}+06$ & $8.51 \mathrm{E}+05$ & $1.11 \mathrm{E}+06$ & $6.57 \mathrm{E}+05$ & $9.32 \mathrm{E}+05$ & $24 \%$ \\
\hline${ }^{99} \mathrm{Tc}$ (IM) & $6.57 \mathrm{E}+05$ & $7.38 \mathrm{E}+05$ & $1.26 \mathrm{E}+06$ & $9.11 \mathrm{E}+05$ & $8.93 \mathrm{E}+05$ & $30 \%$ \\
\hline${ }^{232} \mathrm{Th}$ (IM) & $1.63 \mathrm{E}-01$ & $1.76 \mathrm{E}-01$ & $4.88 \mathrm{E}-01$ & $2.69 \mathrm{E}-01$ & $2.74 \mathrm{E}-01$ & $55 \%$ \\
\hline${ }^{233} \mathrm{U}$ (IM) & $<4.10 \mathrm{E}+03$ & $<4.07 \mathrm{E}+03$ & $<1.57 \mathrm{E}+04$ & $<1.55 \mathrm{E}+04$ & $<9.83 \mathrm{E}+03$ & - \\
\hline${ }^{234} \mathrm{U}$ (IM) & $<2.66 \mathrm{E}+03$ & $<2.64 \mathrm{E}+03$ & $<1.02 \mathrm{E}+04$ & $<1.00 \mathrm{E}+04$ & $<6.38 \mathrm{E}+03$ & - \\
\hline${ }^{235} \mathrm{U}$ (IM) & $1.91 \mathrm{E}+01$ & $1.59 \mathrm{E}+01$ & $2.36 \mathrm{E}+01$ & $1.58 \mathrm{E}+01$ & $1.86 \mathrm{E}+01$ & $20 \%$ \\
\hline${ }^{236} \mathrm{U}$ (IM) & $4.64 \mathrm{E}+01$ & $5.66 \mathrm{E}+01$ & $<1.05 \mathrm{E}+02$ & $<1.04 \mathrm{E}+02$ & $5.15 \mathrm{E}+01$ & $14 \%$ \\
\hline${ }^{237} \mathrm{~Np}$ (IM) & $2.14 \mathrm{E}+03$ & $1.84 \mathrm{E}+03$ & $2.99 \mathrm{E}+03$ & $1.94 \mathrm{E}+03$ & $2.22 \mathrm{E}+03$ & $24 \%$ \\
\hline${ }^{238} \mathrm{U}$ (IM) & $7.62 \mathrm{E}+01$ & $6.48 \mathrm{E}+01$ & $8.11 \mathrm{E}+01$ & $5.36 \mathrm{E}+01$ & $6.89 \mathrm{E}+01$ & $18 \%$ \\
\hline${ }^{239} \mathrm{Pu}$ (IM) & $6.34 \mathrm{E}+05$ & $5.36 \mathrm{E}+05$ & $<2.49 \mathrm{E}+05$ & $<2.46 \mathrm{E}+05$ & $5.85 \mathrm{E}+05$ & $12 \%$ \\
\hline${ }^{240} \mathrm{Pu}$ (IM) & $3.07 \mathrm{E}+05$ & $2.53 \mathrm{E}+05$ & $4.07 \mathrm{E}+05$ & $<3.62 \mathrm{E}+05$ & $3.22 \mathrm{E}+05$ & $24 \%$ \\
\hline
\end{tabular}

Values in italics were not used in calculating the average. Divide $\mathrm{mg} / \mathrm{kg}$ values by $1 \mathrm{E}+04$ to convert to wt $\%$ basis. 


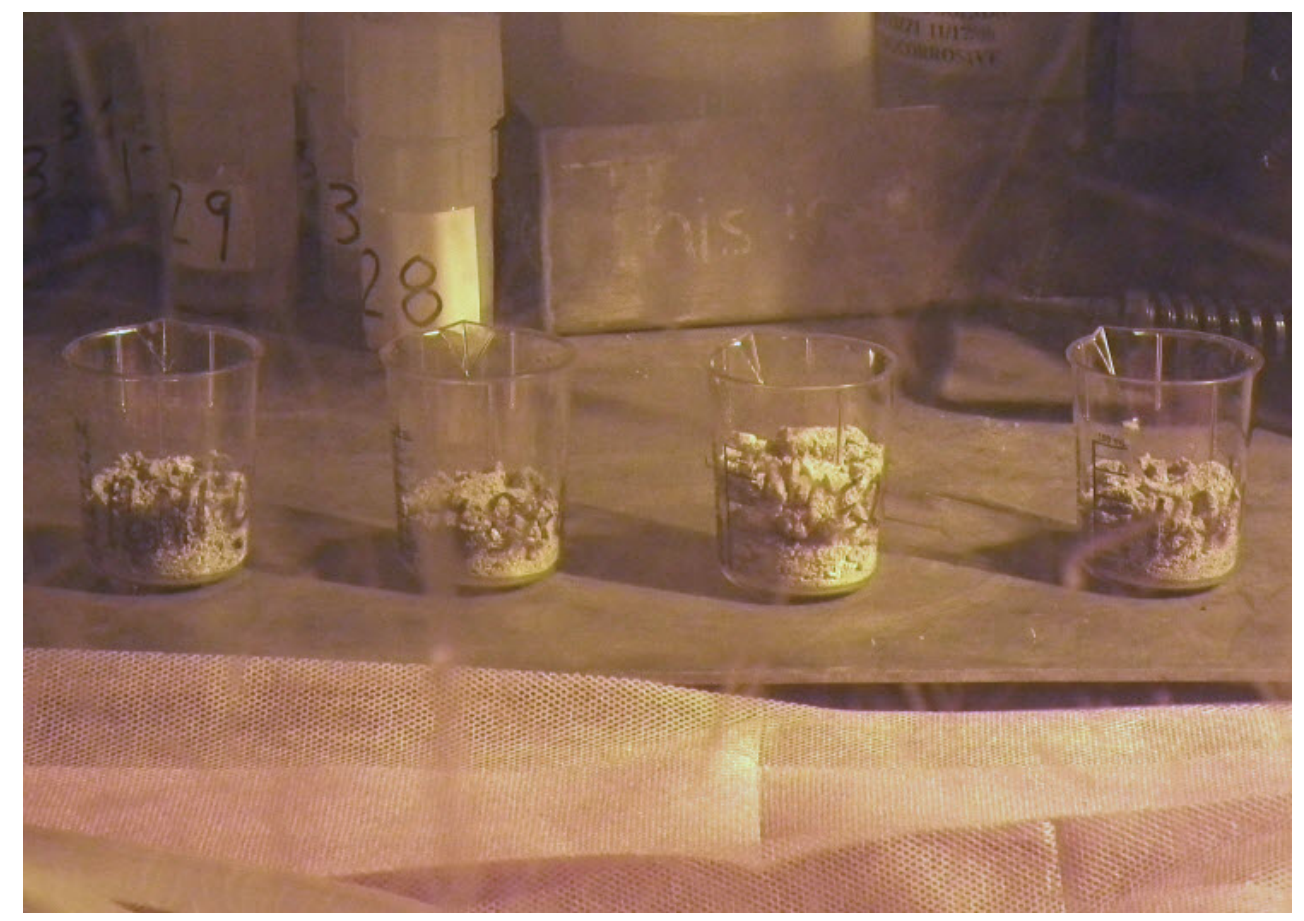

Figure 3-1 Oxalic Acid Leached Solids from the Tank 16H Annulus Samples 


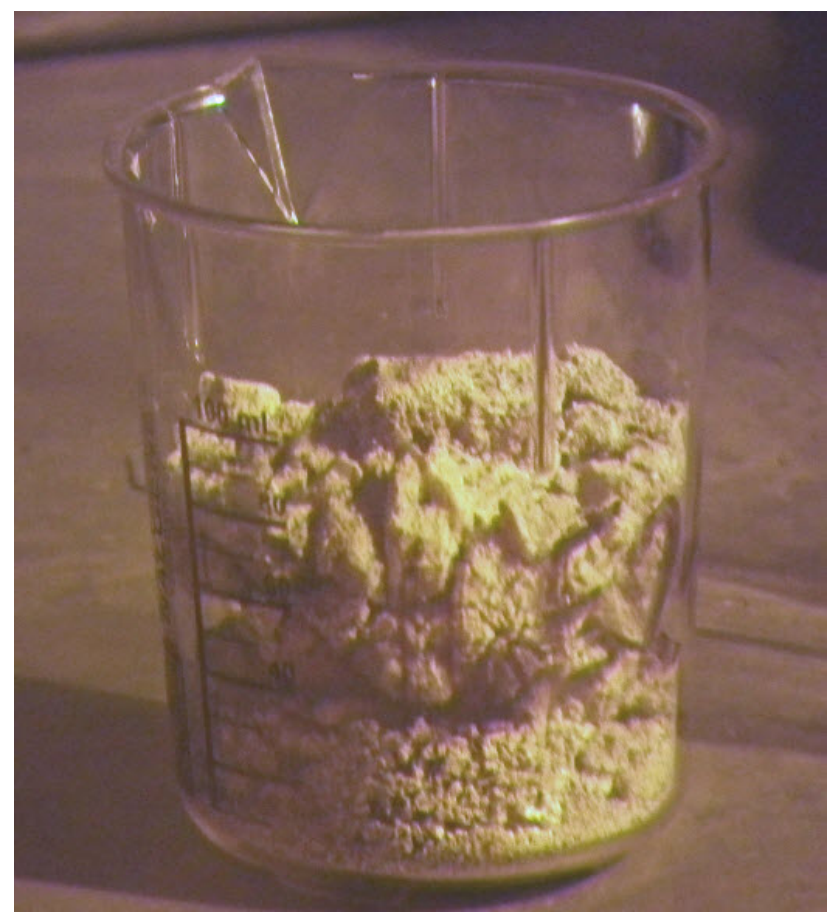

Figure 3-2 Oxalic Acid Leached Solids from Tank 16H Annulus Sample HTF 16-E-2

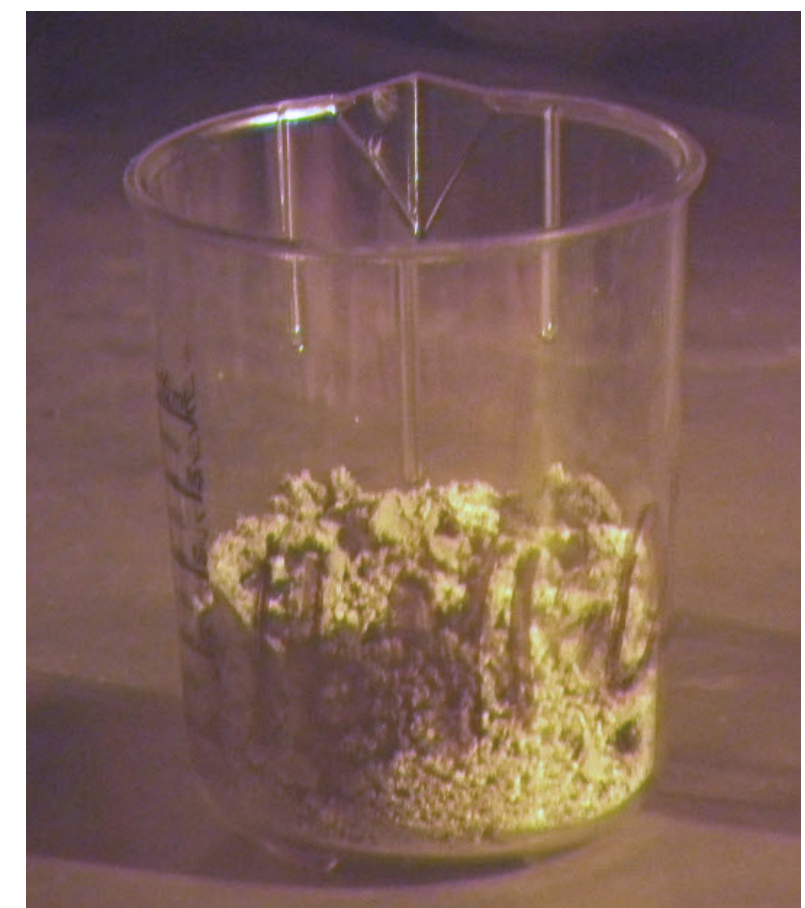

Figure 3-3 Oxalic Acid Leached Solids from Tank 16H Annulus Sample HTF 16-N-1 
Figure 3-4 Oxalic Acid Leached Solids from Tank 16H Annulus Sample HTF 16-S-2

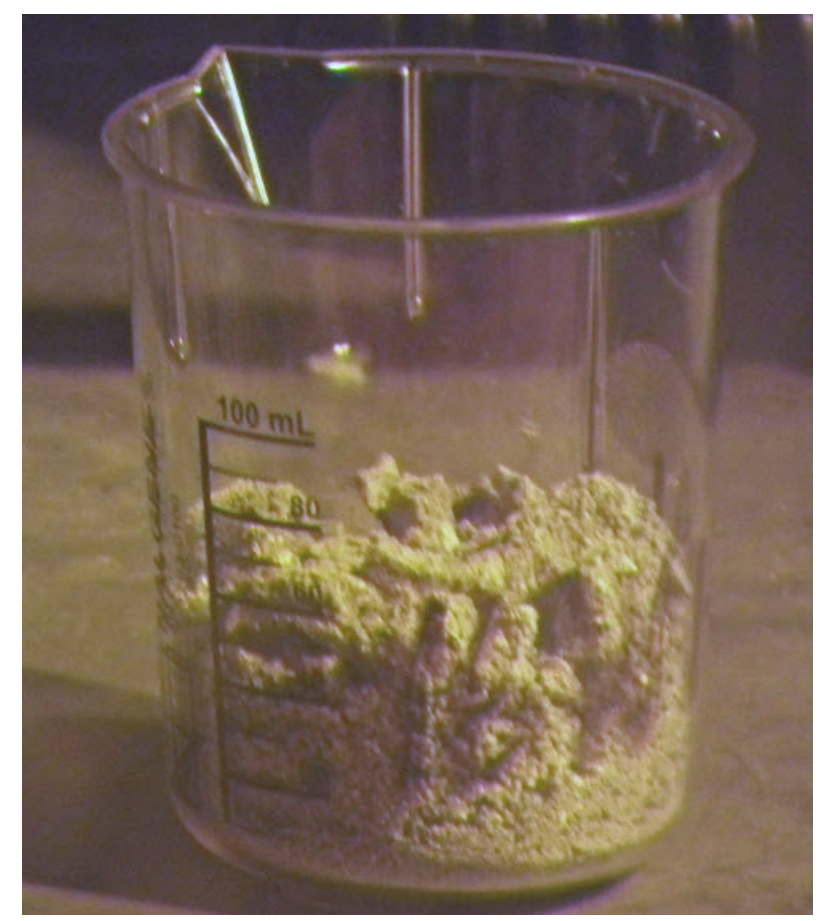

Figure 3-5 Oxalic Acid Leached Solids from Tank 16H Annulus Sample HTF 16-W-1 
Table 3-14. XRD Result for the Oxalic Acid Leached Solids from the Tank 16H Annulus Samples-Estimated Mineral Phase Composition

\begin{tabular}{|c|c|c|c|c|c|c|c|c|}
\hline Crystalline Phase & $\begin{array}{l}\text { HTF-16E2 } \\
\text { Replicate } 1 \\
\end{array}$ & $\begin{array}{l}\text { HTF-16E2 } \\
\text { Replicate } 2\end{array}$ & $\begin{array}{l}\text { HTF-16N1 } \\
\text { Replicate } 1 \\
\end{array}$ & $\begin{array}{l}\text { HTF-16N1 } \\
\text { Replicate } 2\end{array}$ & $\begin{array}{l}\text { HTF-16S2 } \\
\text { Replicate 1 }\end{array}$ & $\begin{array}{l}\text { HTF-16S2 } \\
\text { Replicate } 2\end{array}$ & $\begin{array}{l}\text { HTF-16W1 } \\
\text { Replicate } 1 \\
\end{array}$ & $\begin{array}{l}\text { HTF-16W1 } \\
\text { Replicate } 2\end{array}$ \\
\hline Amorphous & $45 \%$ & $47 \%$ & $10 \%$ & $24 \%$ & $37 \%$ & $45 \%$ & $39 \%$ & $34 \%$ \\
\hline $\mathrm{SiO}_{2}$ & $30 \%$ & $5 \%$ & $78 \%$ & $36 \%$ & $23 \%$ & $35 \%$ & - & - \\
\hline $\mathrm{Na}_{8}\left(\mathrm{Al}_{6} \mathrm{Si}_{6} \mathrm{O}_{24}\right)\left(\mathrm{NO}_{3}\right)_{2} \cdot 4 \mathrm{H}_{2} \mathrm{O}$ & $20 \%$ & $44 \%$ & $10 \%$ & $35 \%$ & $37 \%$ & $13 \%$ & $61 \%$ & $66 \%$ \\
\hline $\mathrm{Al}(\mathrm{OH})_{3}$ & - & - & - & - & $2 \%$ & $5 \%$ & - & - \\
\hline
\end{tabular}


SRNL-STI-2012-00309

Revision 0

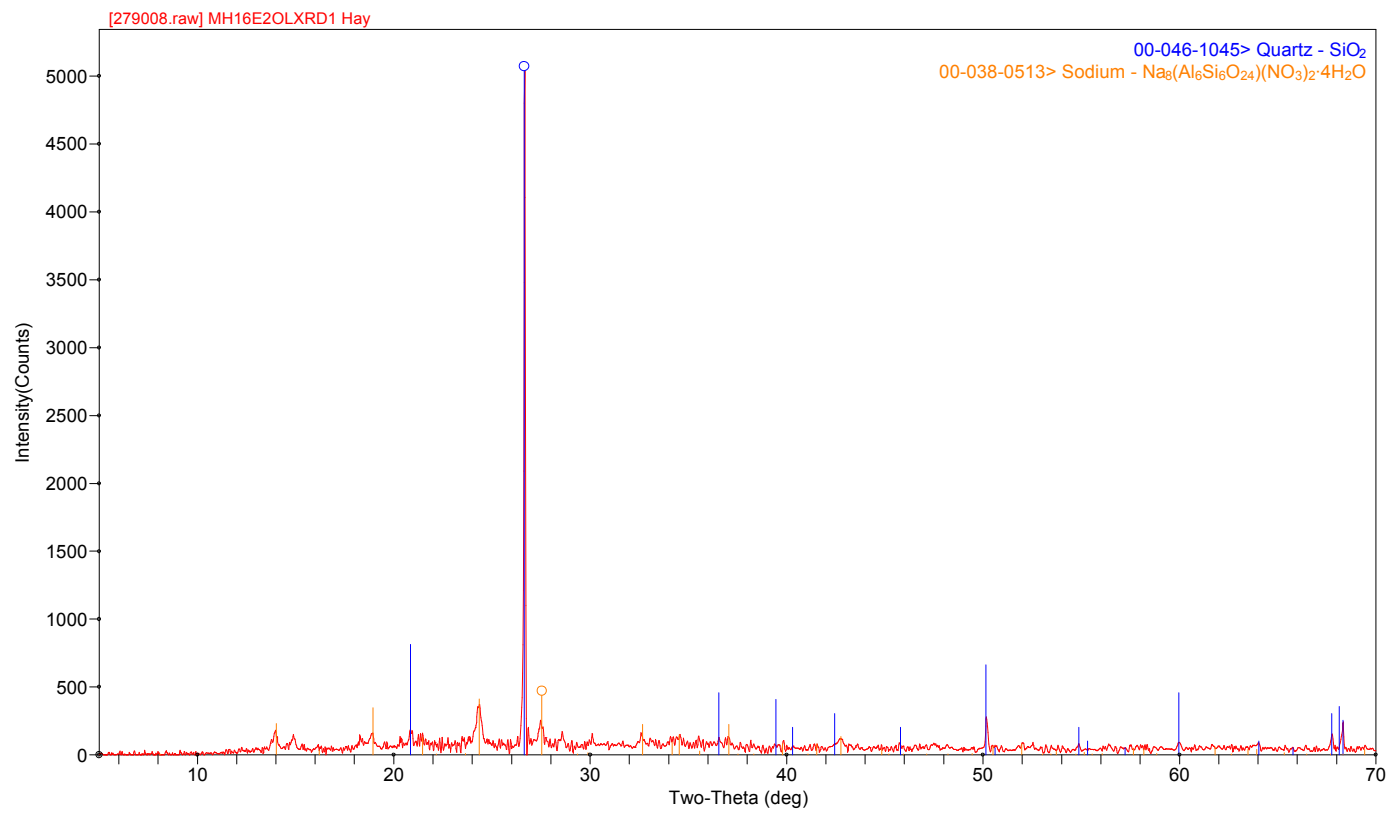

Figure 3-6 XRD Results of the Oxalic Acid Leached Solids for HTF-16E2-1

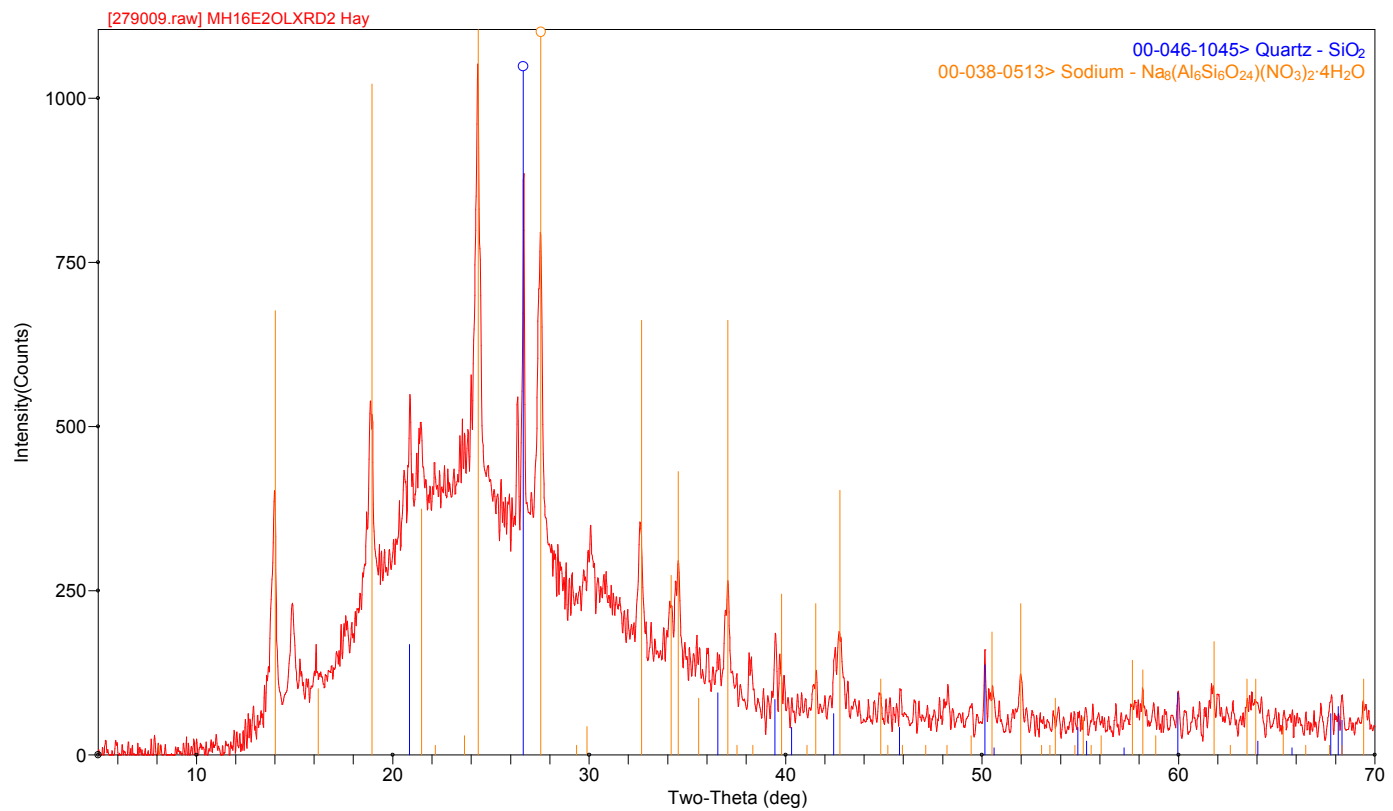

Figure 3-7 XRD Results of the Oxalic Acid Leached Solids for HTF-16E2-2 
SRNL-STI-2012-00309

Revision 0

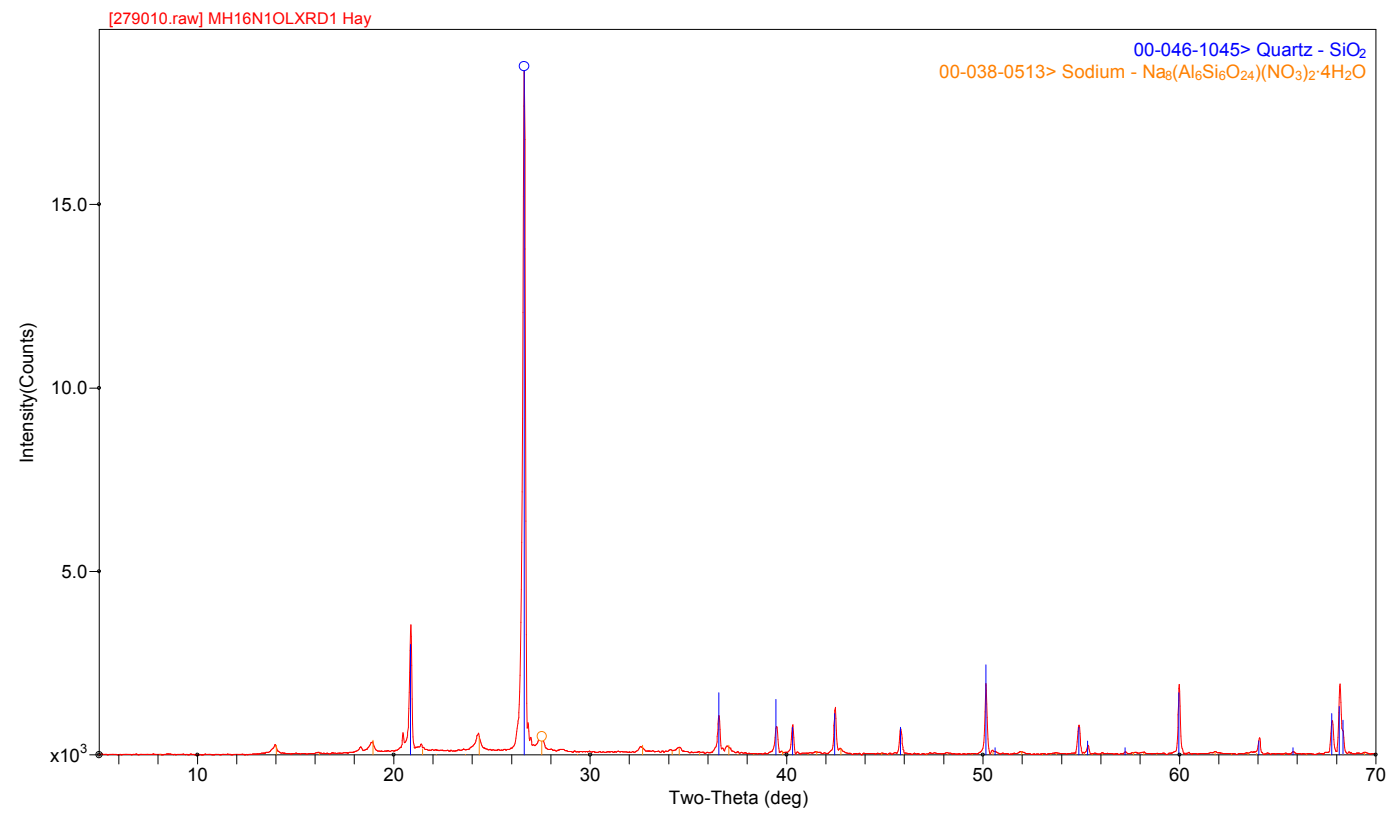

Figure 3-8 XRD Results of the Oxalic Acid Leached Solids for HTF-16N1-1

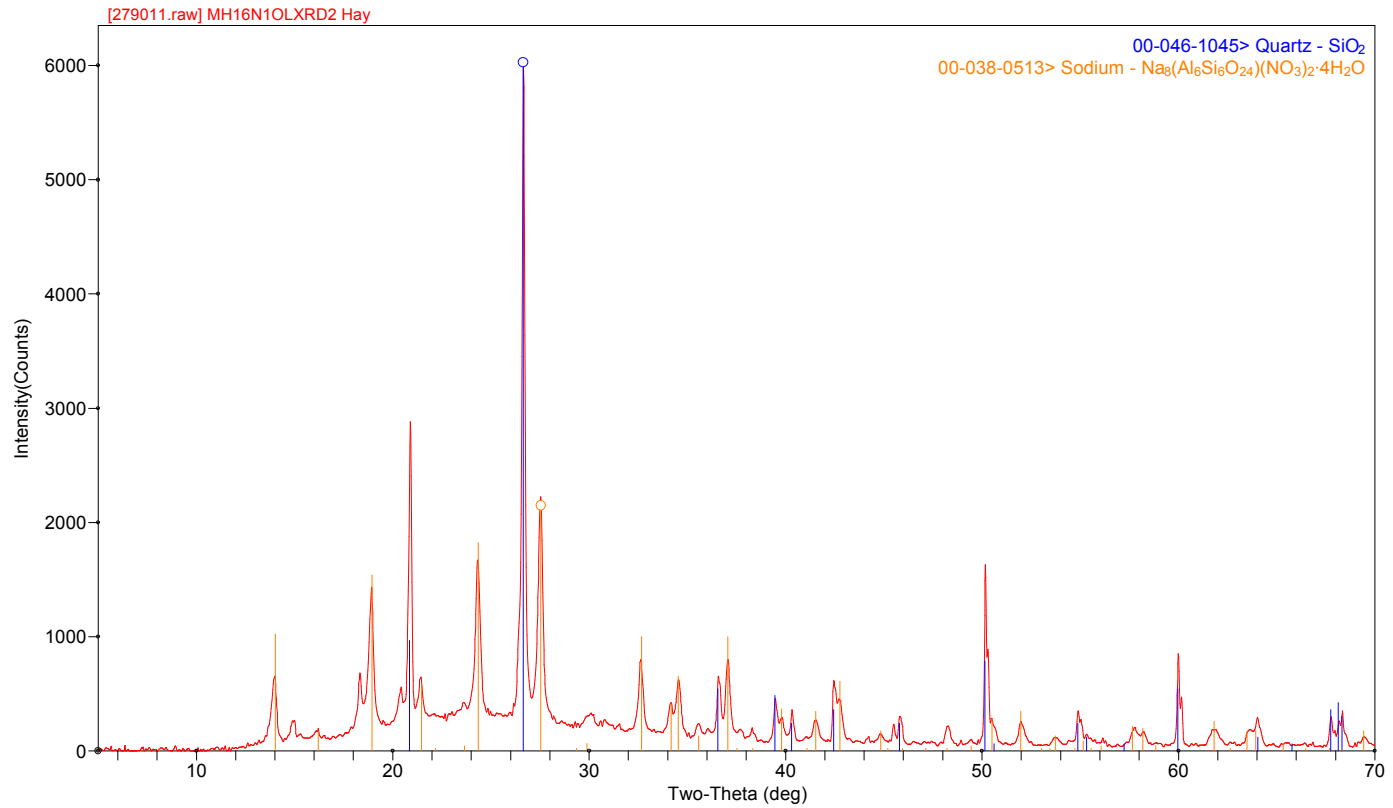

Figure 3-9 XRD Results of the Oxalic Acid Leached Solids for HTF-16N1-2 
SRNL-STI-2012-00309

Revision 0

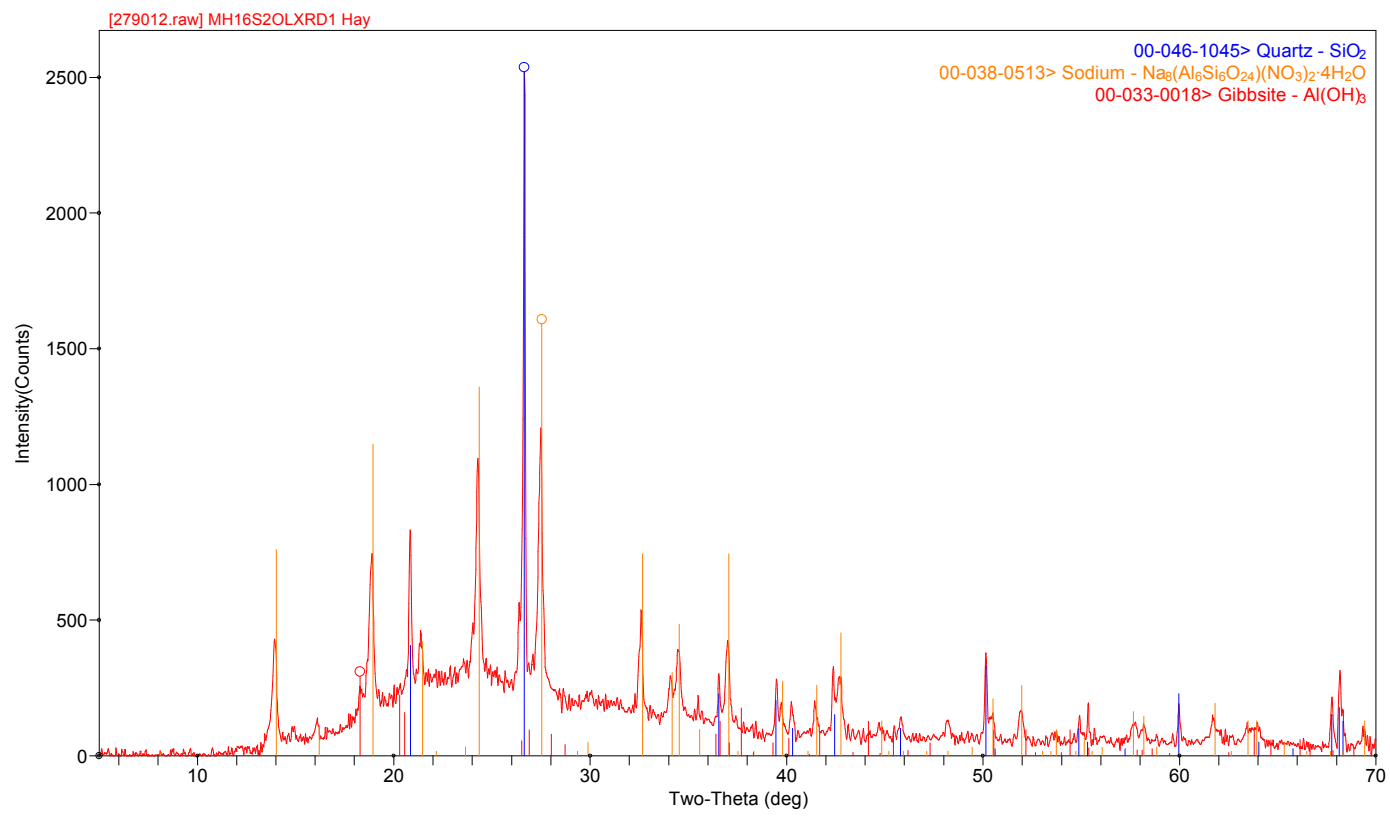

Figure 3-10 XRD Results of the Oxalic Acid Leached Solids for HTF-16S2-1

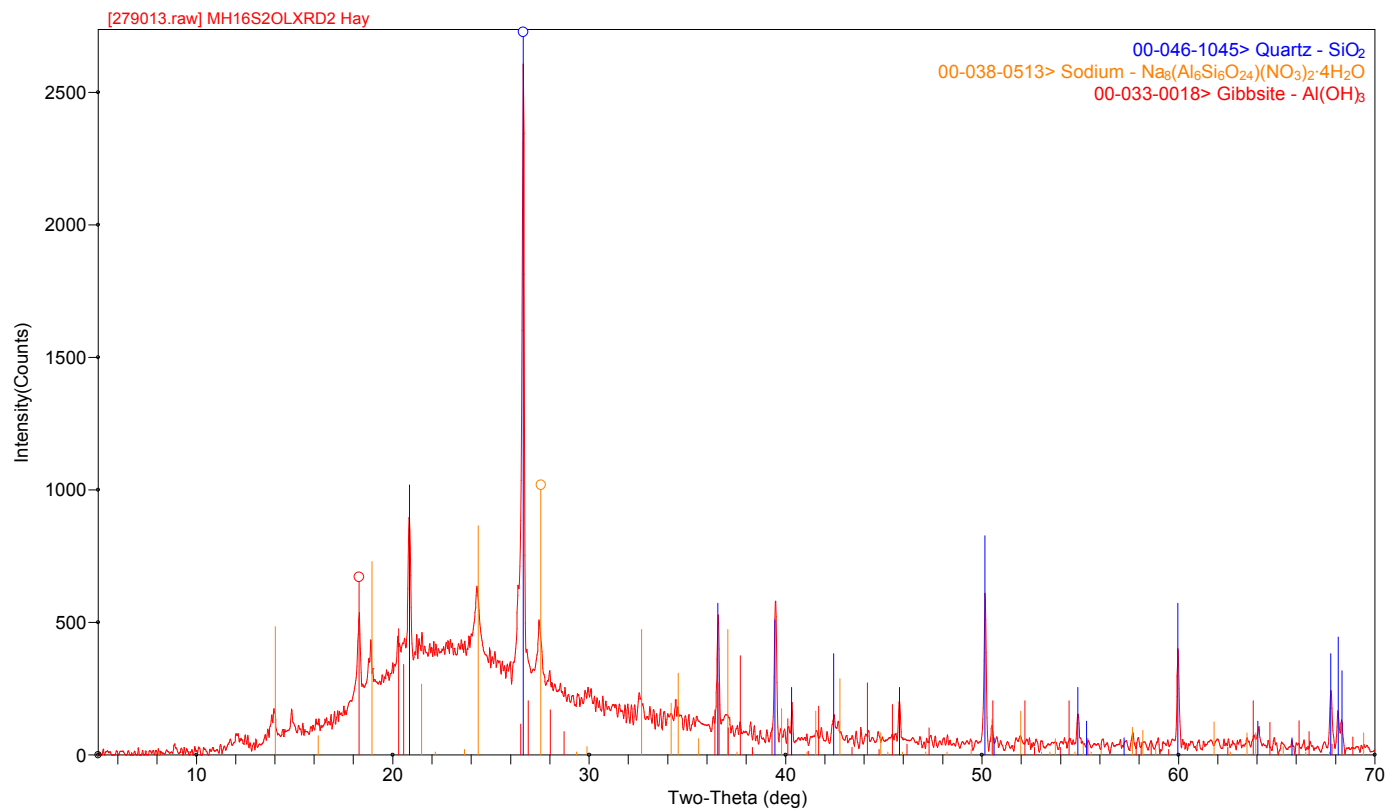

Figure 3-11 XRD Results of the Oxalic Acid Leached Solids for HTF-16S2-2 
SRNL-STI-2012-00309

Revision 0

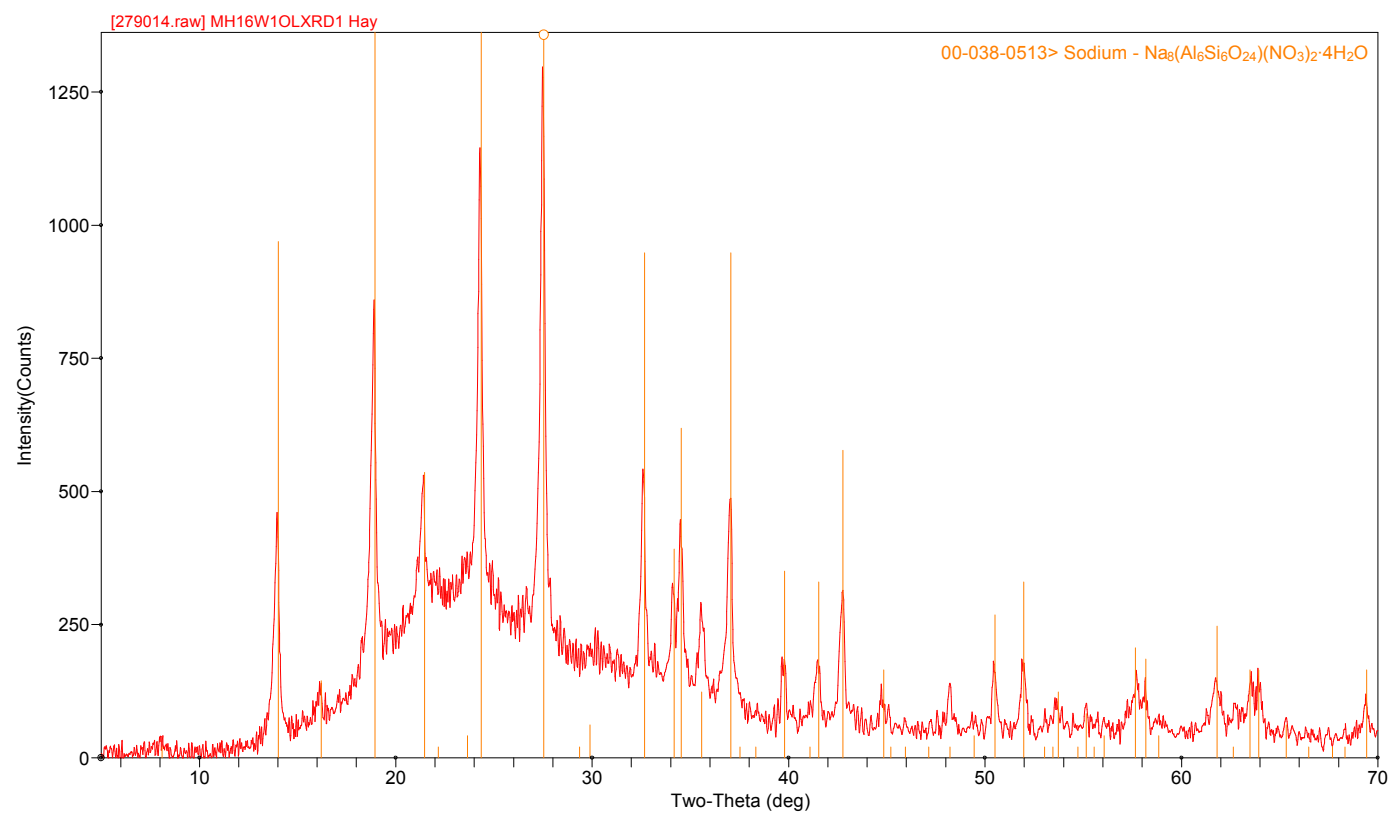

Figure 3-12 XRD Results of the Oxalic Acid Leached Solids for HTF-16W1-1

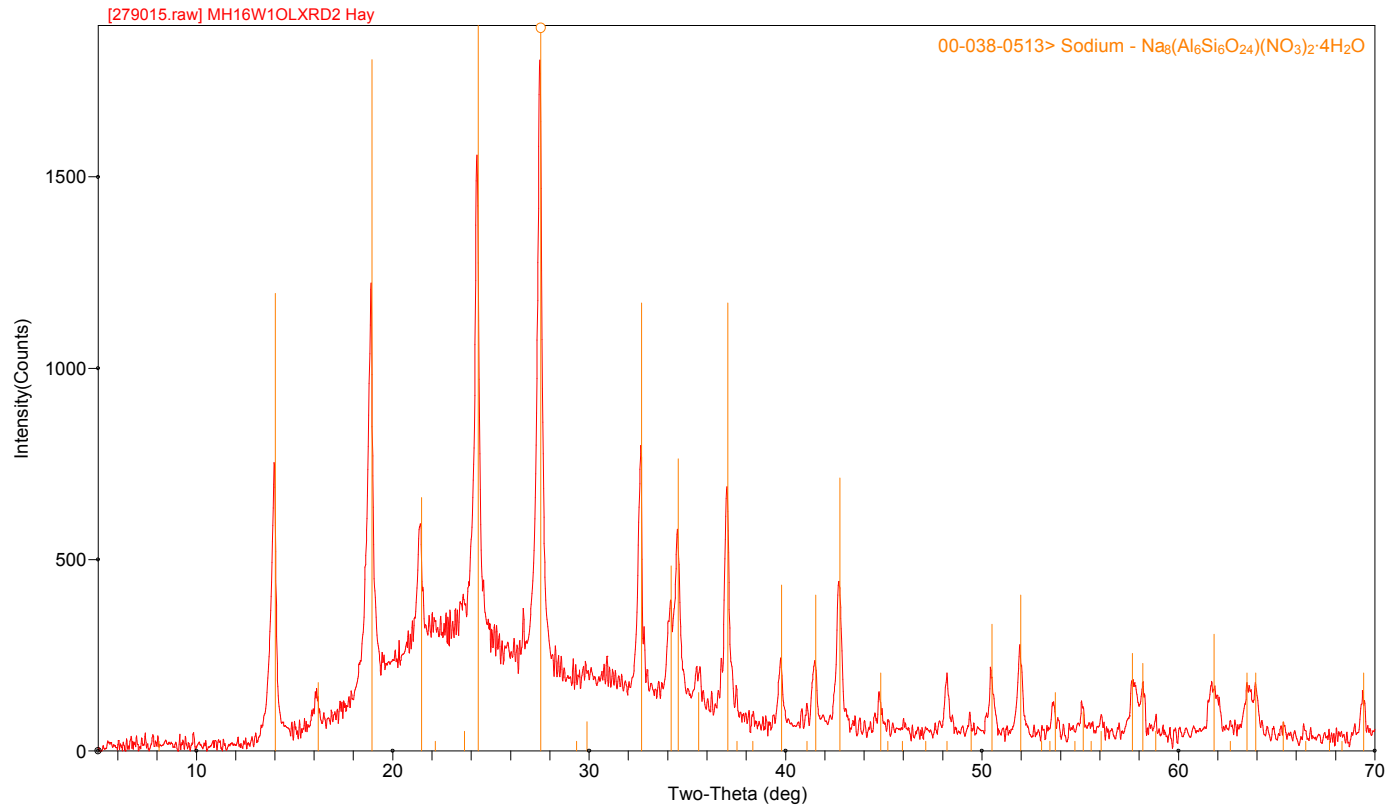

Figure 3-13 XRD Results of the Oxalic Acid Leached Solids for HTF-16W1-2 
SRNL-STI-2012-00309

Revision 0

\subsection{Modeling Summary and Recommendations}

A simple, single leaching cycle model was developed using the OLI Systems Inc, Environmental Simulation Program (ESP), version 8.3, to develop recommendations on the choice of species from the thermodynamic database to represent Tank 16 Annulus solids. The simple model contained a single Mix block to combine a solids only stream of species representing the annulus solids with an aqueous stream representing the leaching solution (either water or $4 \mathrm{wt} \%$ oxalic acid). The chemistry model containing the species shown in Table 4-1 was developed using the Mixed Solvent Electrolyte (MSE) framework. All of the species in the chemistry model were contained in the Mixed Solvent Electrolyte Public (MSEPUB) database.

The MSEPUB database does not contain the sodium aluminum nitrate silicate hydrate $\left(\mathrm{Na}_{8}\left(\mathrm{Al}_{6} \mathrm{Si}_{6} \mathrm{O}_{24}\right)\left(\mathrm{NO}_{3}\right)_{2} \cdot 4 \mathrm{H}_{2} \mathrm{O}\right)$ or the muscovite $\left(\mathrm{KAl}_{2}\left(\mathrm{AlSi}_{3} \mathrm{O}_{10}\right)(\mathrm{OH})_{2}\right)$ identified in the $\mathrm{XRD}$ results of the as-received Tank 16 Annulus solids. However, the MSEPUB database does contain the compounds cancrinite dihydrate $\left(\mathrm{Na}_{8}\left(\mathrm{Al}_{6} \mathrm{Si}_{6} \mathrm{O}_{24}\right)\left(\mathrm{CO}_{3}\right) \cdot 2 \mathrm{H}_{2} \mathrm{O}\right)$ and hydroxysodalite dihydrate $\left(\mathrm{Na}_{8} \mathrm{Al}_{6} \mathrm{Si}_{6} \mathrm{O}_{24}(\mathrm{OH})_{2} .2 \mathrm{H}_{2} \mathrm{O}\right)$ that have similar chemical formulas. Using the simple ESP model, the hydroxysodalite dihydrate was found to better predict the solubility of silicon measured in the water and oxalic acid leaching tests on Tank 16 annulus solids.

Table 4-1. Species Chosen for the ESP Chemistry Model of Tank 16 Annulus Solids

\begin{tabular}{|l|l|}
\hline $\mathrm{H}_{2} \mathrm{O}$ & $\mathrm{Na}_{3} \mathrm{H}\left(\mathrm{CO}_{3}\right)_{2} \cdot 2 \mathrm{H}_{2} \mathrm{O}$ \\
\hline $\mathrm{SiO}_{2}$ & $\mathrm{Na}_{2} \mathrm{CO}_{3}$ \\
\hline $\mathrm{Na}_{8} \mathrm{Al}_{6} \mathrm{Si}_{6} \mathrm{O}_{24}(\mathrm{OH})_{2} \cdot 2 \mathrm{H}_{2} \mathrm{O}$ & $\mathrm{Na}_{2} \mathrm{SO}_{4}$ \\
\hline $\mathrm{Al}(\mathrm{OH})_{3}$ & $\mathrm{NaNO}_{3}$ \\
\hline $\mathrm{Fe}_{2} \mathrm{O}_{3}$ & $\mathrm{NaNO}_{2}$ \\
\hline $\mathrm{Ca}(\mathrm{OH})_{2}$ & $\mathrm{KNO}_{3}$ \\
\hline $\mathrm{CaSO}_{4}$ & $\mathrm{~K}_{2} \mathrm{CO}_{3}$ \\
\hline $\mathrm{CaCO}_{3}$ & $\mathrm{Mg}(\mathrm{OH})_{2}$ \\
\hline $\mathrm{Hg}$ & $\mathrm{H}_{2} \mathrm{C}_{2} \mathrm{O}_{4}$ \\
\hline $\mathrm{HgO}$ & $\mathrm{NaOH}^{\mathrm{NgO}}$ \\
\hline
\end{tabular}

The model predicts that all of the sodium nitrite, sodium nitrate, sodium sulfate, sodium carbonate, and sodium bicarbonate dihydrate (trona) will be completely soluble in water 
using a 3:1 ratio of water volume to solids mass. This prediction agrees well with the water leaching test results. However, comparing the final water leach solution concentrations for the first water contact with the model predictions showed the model was over-predicting the sulfate concentration by $40-50 \%$. This indicates the annulus samples may have some percentage of sulfate that is not soluble in water.

The simple model predictions for the solubility of $\mathrm{Hg}$ and $\mathrm{Si}$ in water match the measured values from the leaching test because the ratios of the more soluble and less soluble forms of $\mathrm{Hg}$ and $\mathrm{Si}$ in the model were adjusted to yield values that matched solubilities measured in the water leaching tests. As it turned out, that division of the Si between the more water soluble hydroxysodalite and the less water soluble silicon dioxide for each sample composition was similar to the percentages of each observed in the XRD results of the as-received solids. The total $\mathrm{Hg}$ concentration of the sample was divided between the more water soluble $\mathrm{HgO}$ and the less water soluble elemental $\mathrm{Hg}$. The model does poorly predicting the solubility of $\mathrm{Al}, \mathrm{Fe}, \mathrm{Ca}, \mathrm{K}$, and $\mathrm{Mg}$ with differences of greater than $+/-2 \mathrm{X}$ compared to the solution concentrations from the leaching test results. However, the model predictions closely matched the total amount of material dissolved in the water leaching test.

The simple model predictions of the oxalic acid leaching test showed large deviations (greater than $+/-2 \mathrm{X}$ ) from the measured solution concentrations in the oxalic acid leaching tests. This difference may result from a thermodynamics issue and the simple model only containing one leaching cycle. The thermodynamic modeling software takes all reactions to equilibrium. However, some of the dissolution reactions of the Tank 16 annulus solids in oxalic acid may require more than the 24 hour time period used in the leaching test to reach equilibrium. The results from the leaching tests indicate more material continued to dissolve from the annulus solids with each oxalic acid contact. Both of these issues indicate the simple model will not be sufficient for predicting the amount of Tank 16 annulus solids dissolved by oxalic acid leaching.

A larger model with three successive leaching/decant steps was developed to better evaluate the ability of the software to predict the leaching test results. This model used the same Mix block followed by a Filter block that allows partitioning of the liquid phase. The slurry output from the Filter block feeds the next Mix block.

The larger, three leaching cycle ESP model appears to predict the dissolution of water soluble components from the Tank 16 Annulus solids with reasonable accuracy but shows large deviations (greater than $+/-2 \mathrm{X}$ ) from the measured solution concentrations for the components with low water solubility. However, since very little of these low water solubility components dissolved in the water leaching test, the large deviations from the measured solution concentrations may be less important than the generalized prediction of low solubility by the model. The model accurately predicted $(<20 \%$ difference) the total amount of material dissolved from the annulus solids after three leaching cycles. 
In modeling the oxalic acid leaching test results, the larger model showed large deviations (greater than $+/-2 \mathrm{X}$ ) from the measured solution concentrations for each leaching cycle even for the most water soluble components. However, the model correctly predicted which components would exhibit high and low solubility in the oxalic acid and predicted the total amount of material dissolved from the annulus solids within $20 \%$ for the one sample examined.

Even given the inaccuracies in the model, it can be of some benefit in planning and evaluating processing options. Recommendations for modeling the leaching of Tank 16 Annulus solids include;

- Use the species shown in Table 4-1 for the chemistry model and the MSE framework. However, evaluation of other $\mathrm{Hg}$ species may provide better results for $\mathrm{Hg}$ solubility predictions.

- Develop a model construction that allows the simulation of three successive oxalic acid contacts since the tests showed that additional material will dissolve from the Tank 16 Annulus solids with each contact. 
SRNL-STI-2012-00309

Revision 0

\subsection{Conclusions}

Table 5-1 summarizes the composition of the major components of the as-received annulus samples, the water leached solids, and the oxalic acid leached solids. The analysis found all four annulus samples to be composed mainly of $\mathrm{Si}, \mathrm{Na}, \mathrm{Al}$, and $\mathrm{Fe}$ and lesser amounts of other elements both before and after leaching with either water or oxalic acid. The solid-phase sodium concentration decreases with both water and oxalic acid leaching, due to dissolution of dominant sodium salts such as $\mathrm{NaNO}_{3}$ and $\mathrm{NaNO}_{2}$. The silicon concentration in the solids remaining after leaching increases substantially since very little of the silicon containing compounds dissolve in either leaching solution. With water leaching, the data indicate that most of the material that will be leached dissolves in the first water contact. Subsequent water contacts merely serve to dilute the concentrations of the dissolved solids in the interstitial liquid. ${ }^{3}$ The oxalic acid leaching results indicate that material from the annulus continues to dissolve with each new contact. The $\mathrm{pH}$ of the solution drops with each addition of oxalic acid persuading more material to dissolve.

Table 5-1. Summary of the Composition of the As-Received, Water Leached, and Oxalic Acid Leached Solids from Tank 16H Annulus Samples (Wt\% Total Dried Solids)

\begin{tabular}{|l|c|c|c|c|c|c|c||}
\hline \hline Sample ID & $\begin{array}{c}\text { Si } \\
\mathbf{w t} \%\end{array}$ & $\begin{array}{c}\text { Na } \\
\mathbf{w t} \%\end{array}$ & $\begin{array}{c}\text { Al } \\
\mathbf{w t} \%\end{array}$ & $\begin{array}{c}\mathbf{F e} \\
\mathbf{w t} \%\end{array}$ & $\begin{array}{c}\mathbf{C a} \\
\mathbf{w t} \%\end{array}$ & $\begin{array}{c}\text { S } \\
\mathbf{w t} \%\end{array}$ & $\begin{array}{c}\text { Hg } \\
\mathbf{w t} \%\end{array}$ \\
\hline HTF-16-E-2 & $17.0 \%$ & $12.1 \%$ & $6.62 \%$ & $6.54 \%$ & $1.58 \%$ & $0.14 \%$ & $0.21 \%$ \\
\hline HTF-16-E-2 WL & $21.1 \%$ & $9.50 \%$ & $9.73 \%$ & $3.68 \%$ & $1.57 \%$ & $0.06 \%$ & $0.18 \%$ \\
\hline HTF-16-E-2 OAL & $23.5 \%$ & $4.78 \%$ & $6.82 \%$ & $3.47 \%$ & $1.58 \%$ & $0.06 \%$ & $0.13 \%$ \\
\hline & & & & & & & \\
\hline HTF-16-N-1 & $21.1 \%$ & $12.1 \%$ & $7.27 \%$ & $1.12 \%$ & $0.61 \%$ & $0.16 \%$ & $0.10 \%$ \\
\hline HTF-16-N-1 WL & $25.1 \%$ & $10.8 \%$ & $9.47 \%$ & $0.79 \%$ & $0.22 \%$ & $<0.05 \%$ & $0.07 \%$ \\
\hline HTF-16-N-1 OAL & $21.5 \%$ & $5.13 \%$ & $9.53 \%$ & $1.31 \%$ & $0.37 \%$ & $0.06 \%$ & $0.16 \%$ \\
\hline & & & & & & & \\
\hline HTF-16-S-2 & $17.1 \%$ & $15.9 \%$ & $4.20 \%$ & $2.01 \%$ & $0.47 \%$ & $0.55 \%$ & $0.35 \%$ \\
\hline HTF-16-S-2 WL & $20.6 \%$ & $7.32 \%$ & $8.05 \%$ & $2.84 \%$ & $0.37 \%$ & $0.09 \%$ & $0.27 \%$ \\
\hline HTF-16-S-2 OAL & $20.2 \%$ & $3.31 \%$ & $5.19 \%$ & $2.28 \%$ & $0.39 \%$ & $0.24 \%$ & $0.37 \%$ \\
\hline & & & & & & & \\
\hline HTF-16-W-1 & $11.7 \%$ & $17.9 \%$ & $8.35 \%$ & $3.58 \%$ & $0.14 \%$ & $0.14 \%$ & $0.26 \%$ \\
\hline HTF-16-W-1 WL & $17.5 \%$ & $12.1 \%$ & $12.30 \%$ & $2.46 \%$ & $1.19 \%$ & $<0.05 \%$ & $0.25 \%$ \\
\hline HTF-16-W-1 OAL & $26.2 \%$ & $9.25 \%$ & $9.61 \%$ & $1.73 \%$ & $0.09 \%$ & $0.09 \%$ & $0.20 \%$ \\
\hline Samplo & & & & & & \\
\hline
\end{tabular}

Sample ID's ending in -WL are from the water leach and those ending in -OAL are from the oxalic acid leach./ 
Based on the weight of solids used in each test and the weight of solids remaining at the end of the test, Table 5-2 indicates $20-35 \%$ of the solids dissolved after three water contacts with an approximately $3: 1$ volume of water to solids at $45{ }^{\circ} \mathrm{C}$. The oxalic acid leaching tests dissolved slightly more material ranging from $34 \%-47 \%$ with an approximately $3: 1$ volume of oxalic acid to solids at $45^{\circ} \mathrm{C}$. With both leaching solutions sample HTF-16-E-2 showed the least dissolution and samples HTF-16-S-2 and HTF-16$\mathrm{W}-1$ showed the highest percentage dissolution.

Table 5-2. Estimated Percent of Solids Dissolved in the Water Leaching and Oxalic Acid Leaching Tests on Tank 16H Annulus Samples

\begin{tabular}{||l|c|c||}
\hline Sample ID & $\begin{array}{c}\text { Estimated Percent } \\
\text { Dissolved in Water } \\
\text { Leach }\end{array}$ & $\begin{array}{c}\text { Estimated Percent } \\
\text { Dissolved in Oxalic } \\
\text { Acid Leach }\end{array}$ \\
\hline HTF-16-E-2 & $20 \%$ & $34 \%$ \\
\hline HTF-16-N-1 & $29 \%$ & $38 \%$ \\
\hline HTF-16-S-2 & $35 \%$ & $46 \%$ \\
\hline HTF-16-W-1 & $33 \%$ & $47 \%$ \\
\hline
\end{tabular}

Tables 5-3 and 5-4 provide summaries of the leaching behavior of the radionuclides analyzed in the samples from the water leaching and oxalic acid leaching tests. The estimated percent removal values were calculated from the concentration of the radionuclide in the as-received solids minus the estimated amount removed in each leaching cycle. The percentage removal values can only be estimated since the amount decanted varied based on the settling characteristics of the sample. For the calculations, $100 \mathrm{~mL}$ of the first decant and $150 \mathrm{~mL}$ of the second and third decants were assumed to be removed. The estimated values from the water leaching differ slightly from the previously reported values because they include material from all three water contacts instead of just the first water contact. The values for the estimated percent of solids dissolved in the water leaching presented in the previous report only included the material removed in the first leaching cycle. ${ }^{3}$ The total strontium results from the ICP-ES method provide insight into the behavior for ${ }^{90} \mathrm{Sr}$. The oxalic acid leach was somewhat more effective at removing the radionuclides from the annulus solids than the water leach. 
SRNL-STI-2012-00309

Revision 0

Table 5-3. Summary of the Estimated Percentage Dissolved for each Radionuclide from the Water Leaching Test on Tank 16H Annulus Samples

\begin{tabular}{|l|c|c|c|c||}
\hline Radionuclide & HTF-16-E-2 & HTF-16-N-1 & HTF-16-S-2 & HTF-16-W-1 \\
\hline Cs-137 & $2.8 \%$ & $3.1 \%$ & $5.7 \%$ & $3.6 \%$ \\
\hline Sr & $<0.2 \%$ & $<0.9 \%$ & $<0.5 \%$ & $0.7 \%$ \\
\hline Tc-99 & $35 \%$ & $32 \%$ & $34 \%$ & $29 \%$ \\
\hline U-235 & $56 \%$ & $52 \%$ & $16 \%$ & $8.1 \%$ \\
\hline U-238 & $53 \%$ & $58 \%$ & $16 \%$ & $7.9 \%$ \\
\hline Np-237 & $31 \%$ & $16 \%$ & $<8.5 \%$ & $7.5 \%$ \\
\hline Pu-239 & $20 \%$ & $4.1 \%$ & $<1.9 \%$ & $<0.4 \%$ \\
\hline
\end{tabular}

Table 5-4. Summary of the Estimated Percentage Dissolved for each Radionuclide from the Oxalic Acid Leaching Test on Tank 16H Annulus Samples

\begin{tabular}{|l|c|c|c|c||}
\hline \hline Radionuclide & HTF-16-E-2 & HTF-16-N-1 & HTF-16-S-2 & HTF-16-W-1 \\
\hline Cs-137 & $34 \%$ & $38 \%$ & $57 \%$ & $31 \%$ \\
\hline Sr & $11 \%$ & $21 \%$ & $15 \%$ & $29 \%$ \\
\hline Tc-99 & $38 \%$ & $54 \%$ & $49 \%$ & $40 \%$ \\
\hline U-235 & $67 \%$ & $98 \%$ & $67 \%$ & $67 \%$ \\
\hline U-238 & $64 \%$ & $103 \%$ & $72 \%$ & $64 \%$ \\
\hline Np-237 & $35 \%$ & $58 \%$ & $49 \%$ & $53 \%$ \\
\hline Pu-239 & $15 \%$ & $46 \%$ & $34 \%$ & $22 \%$ \\
\hline \hline
\end{tabular}


The chemical analysis of the leachates and the XRD results of the remaining solids indicate sodium salts of nitrate, nitrite, sulfate, and possibly carbonate/bicarbonate make up the majority of the dissolved material with both leaching solutions. The oxalic acid leach also dissolved some fraction of the aluminum in the samples. Based on the results of the leachate analysis, the majority of these salts were dissolved in the first water contact and simply diluted with each subsequent water contact. However, in the oxalic acid leach, material continued to dissolve with each subsequent contact. The $\mathrm{pH}$ of the oxalic acid solutions continued to drop with each contact. Solution concentrations from water and oxalic acid leaching in the annulus can be expected to be lower than found in these small scale tests due to the monolithic structure of the waste in the annulus resulting in lower available surface area.

The somewhat higher dissolution found in the oxalic acid leaching test versus the water leaching test might be offset by the tendency of the oxalic acid solutions to take on a gellike consistency. The filtered solids left behind after three oxalic acid contacts were sticky and formed large clumps after drying. These two observations could indicate potential processing difficulties with solutions and solids from oxalic acid leaching. The gel formation might be avoided by using larger volumes of the acid.

\subsection{Recommendation}

Further testing would be recommended before using oxalic acid to dissolve the Tank $16 \mathrm{H}$ annulus waste to ensure no processing difficulties are encountered in the full scale process. 
SRNL-STI-2012-00309

Revision 0

\subsection{References}

1. M. S. Hay, S. H. Reboul, "Task Technical and Quality Assurance Plan for the Analysis of Tank 16 Annulus Process Samples", SRNL-RP-2011-01169, Rev. 1, November 14, 2011.

2. Technical Task Request, HLE-TTR-2011-010, C. B. Sherburne. November 14, 2011.

3. M. S. Hay, S. H. Reboul, "Characterization of Tank 16 Annulus Samples”, SRNLSTI-2012-00178, Rev. 0, April, 2012.

4. M. S. Hay, "Characterization of Samples from Tank 16H Annulus", WSRC-STI2008-00203, Rev. 0, May 2008.

5. M. S. Hay, "Results of the Characterization and Dissolution Tests of Samples from Tank 16H”, WSRC-RP-99-00124, Rev. 0, January 19, 1999.

6. L. Reynolds, "Summary of Current Data on Tank 16H Annulus Samples", DPST79-360, January 9, 1979.

7. C. J. Coleman, R. A. Dewberry, M. F. Bryant, J. J. Gemmill, "SRL's Performance in Round Robin \#6 - Analysis of Simulated Defense Waste Glass", WSRC-TR-91187, Rev. 0, May 31, 1991.

8. M. S. Hay, T. B. Edwards, "Statistical Analysis of ESP Verification Test Samples", WSRC-RP-94-1224, Rev. 0, November 4, 1994. 
SRNL-STI-2012-00309

Revision 0

Appendix A 
SRNL-STI-2012-00309

Revision 0

Table A-1. Composition of Blanks and Standards for the Water Leached Solids from Tank 16H Annulus Sample

\begin{tabular}{|c|c|c|c|c|c|}
\hline Analyte (Method) & $\begin{array}{c}\text { Aqua Regia } \\
\text { Blank } \\
\text { (mg/kg) }\end{array}$ & $\begin{array}{c}\text { Per Fusion } \\
\text { Blank } \\
(\mathrm{mg} / \mathrm{kg}) \\
\end{array}$ & $\begin{array}{c}\text { Aqua Regia } \\
\text { Glass Std } \\
\text { (mg/kg) }\end{array}$ & $\begin{array}{c}\text { Per Fusion } \\
\text { Glass Std } \\
\text { (mg/kg) }\end{array}$ & $\begin{array}{c}\text { Glass Std } \\
\text { Composition } \\
(\mathrm{mg} / \mathrm{kg})\end{array}$ \\
\hline $\mathrm{Ag}(\mathrm{IE})$ & $<1.15 \mathrm{E}+02$ & $<1.15 \mathrm{E}+03$ & $<1.12 \mathrm{E}+02$ & $<1.15 E+03$ & - \\
\hline $\mathrm{Al}$ (IE) & $5.11 \mathrm{E}+01$ & $2.98 \mathrm{E}+02$ & $2.44 \mathrm{E}+04$ & $2.52 \mathrm{E}+04$ & $2.50 \mathrm{E}+04$ \\
\hline B (IE) & $<1.62 \mathrm{E}+01$ & $<1.62 \mathrm{E}+02$ & $2.63 \mathrm{E}+04$ & $2.54 \mathrm{E}+04$ & $2.69 \mathrm{E}+04$ \\
\hline $\mathrm{Ba}$ (IE) & $<4.16 \mathrm{E}+00$ & $<4.16 \mathrm{E}+01$ & $8.27 \mathrm{E}+02$ & $7.99 \mathrm{E}+02$ & $7.88 \mathrm{E}+02$ \\
\hline Be (IE) & $<4.80 \mathrm{E}-01$ & $<6.40 \mathrm{E}+00$ & $2.19 \mathrm{E}+01$ & $2.40 \mathrm{E}+01$ & - \\
\hline $\mathrm{Ca}$ (IE) & $3.82 \mathrm{E}+01$ & $3.49 \mathrm{E}+03$ & $1.08 \mathrm{E}+04$ & $1.33 \mathrm{E}+04$ & $1.02 \mathrm{E}+04$ \\
\hline $\mathrm{Cd}$ (IE) & $<2.48 \mathrm{E}+00$ & $<2.48 \mathrm{E}+01$ & $4.44 \mathrm{E}+00$ & $<2.48 E+01$ & - \\
\hline Ce (IE) & $<5.66 \mathrm{E}+01$ & $<5.66 \mathrm{E}+02$ & $<5.50 \mathrm{E}+01$ & $<5.66 E+02$ & - \\
\hline Co (IE) & $<7.76 \mathrm{E}+00$ & $<7.76 \mathrm{E}+01$ & $6.54 \mathrm{E}+01$ & $<7.76 E+01$ & - \\
\hline $\mathrm{Cr}$ (IE) & $<6.56 \mathrm{E}+00$ & $<8.16 \mathrm{E}+01$ & $7.23 \mathrm{E}+02$ & $6.49 \mathrm{E}+02$ & $6.36 \mathrm{E}+02$ \\
\hline $\mathrm{Cu}$ (IE) & $<5.04 \mathrm{E}+00$ & $<5.04 \mathrm{E}+01$ & $1.87 \mathrm{E}+01$ & $7.20 \mathrm{E}+01$ & $3.20 \mathrm{E}+01$ \\
\hline $\mathrm{Fe}$ (IE) & $1.84 \mathrm{E}+02$ & $2.65 \mathrm{E}+02$ & $9.89 \mathrm{E}+04$ & $9.74 \mathrm{E}+04$ & $9.79 \mathrm{E}+04$ \\
\hline Gd (IE) & $<1.72 \mathrm{E}+01$ & $<1.72 \mathrm{E}+02$ & $<1.67 \mathrm{E}+01$ & $<1.72 E+02$ & - \\
\hline K (IE) & $<2.05 \mathrm{E}+02$ & $3.93 \mathrm{E}+03$ & $2.13 \mathrm{E}+04$ & $2.65 \mathrm{E}+04$ & $2.25 \mathrm{E}+04$ \\
\hline La (IE) & $<5.36 \mathrm{E}+00$ & $<5.36 \mathrm{E}+01$ & $6.30 \mathrm{E}+00$ & $<5.36 E+01$ & - \\
\hline Li (IE) & $<1.17 \mathrm{E}+01$ & $<1.17 \mathrm{E}+02$ & $1.46 \mathrm{E}+04$ & $1.44 \mathrm{E}+04$ & $1.49 \mathrm{E}+04$ \\
\hline $\mathrm{Mg}$ (IE) & $6.16 \mathrm{E}+00$ & $1.84 \mathrm{E}+01$ & $5.19 \mathrm{E}+03$ & $5.28 \mathrm{E}+03$ & $5.19 \mathrm{E}+03$ \\
\hline Mn (IE) & $<4.24 \mathrm{E}+00$ & $<1.68 \mathrm{E}+01$ & $1.46 \mathrm{E}+04$ & $1.44 \mathrm{E}+04$ & $1.46 \mathrm{E}+04$ \\
\hline Mo (IE) & $<4.94 \mathrm{E}+01$ & $<2.14 \mathrm{E}+02$ & $<4.80 \mathrm{E}+01$ & $<2.14 E+02$ & - \\
\hline $\mathrm{Na}$ (IE) & $<5.90 \mathrm{E}+02$ & - & $8.30 \mathrm{E}+04$ & - & $8.53 \mathrm{E}+04$ \\
\hline Ni (IE) & $<3.64 \mathrm{E}+01$ & $<3.64 \mathrm{E}+02$ & $7.89 \mathrm{E}+03$ & $7.99 \mathrm{E}+03$ & $8.25 \mathrm{E}+03$ \\
\hline $\mathrm{P}$ (IE) & $<6.93 \mathrm{E}+01$ & $<6.93 \mathrm{E}+02$ & $1.10 \mathrm{E}+03$ & $1.03 \mathrm{E}+03$ & $9.60 \mathrm{E}+02$ \\
\hline $\mathrm{Pb}$ (IE) & $<5.73 \mathrm{E}+01$ & $<5.73 \mathrm{E}+02$ & $<5.57 \mathrm{E}+01$ & $<5.73 E+02$ & - \\
\hline $\mathrm{S}$ (IE) & $<6.00 \mathrm{E}+02$ & $<6.00 \mathrm{E}+03$ & $6.44 \mathrm{E}+02$ & $<6.00 E+03$ & - \\
\hline $\mathrm{Sb}$ (IE) & $<1.02 \mathrm{E}+02$ & $<8.53 \mathrm{E}+02$ & $1.52 \mathrm{E}+02$ & $<8.53 E+02$ & - \\
\hline Si (IE) & - & $<7.82 \mathrm{E}+02$ & - & $2.33 \mathrm{E}+05$ & $2.24 \mathrm{E}+05$ \\
\hline Sn (IE) & $<4.49 \mathrm{E}+01$ & $<4.49 \mathrm{E}+02$ & $7.55 \mathrm{E}+01$ & $<4.49 E+02$ & - \\
\hline
\end{tabular}

Values in italics were not used in calculating the average. Divide $\mathrm{mg} / \mathrm{kg}$ values by $1 \mathrm{E}+04$ to convert to wt $\%$ basis. 
SRNL-STI-2012-00309

Revision 0

Table A-1. Composition of Blanks and Standards for the Water Leached Solids from Tank 16H Annulus Sample (continued)

\begin{tabular}{|l|c|c|c|c|c||}
\hline Analyte (Method) & $\begin{array}{c}\text { Aqua Regia } \\
\text { Blank } \\
\text { (mg/kg) }\end{array}$ & $\begin{array}{c}\text { Per Fusion } \\
\text { Blank } \\
\text { (mg/kg) }\end{array}$ & $\begin{array}{c}\text { Aqua Regia } \\
\text { Glass Std } \\
\text { (mg/kg) }\end{array}$ & $\begin{array}{c}\text { Per Fusion } \\
\text { Glass Std } \\
\text { (mg/kg) }\end{array}$ & $\begin{array}{c}\text { Glass Std } \\
\text { Composition } \\
\text { (mg/kg) }\end{array}$ \\
\hline Sr (IE) & $<4.00 \mathrm{E}-01$ & $3.52 \mathrm{E}+01$ & $3.21 \mathrm{E}+01$ & $6.72 \mathrm{E}+01$ & $3.13 \mathrm{E}+01$ \\
\hline Th (IE) & $<4.28 \mathrm{E}+01$ & $<4.38 \mathrm{E}+02$ & $<4.16 \mathrm{E}+01$ & $<4.38 \mathrm{E}+02$ & - \\
\hline Ti (IE) & $<1.04 \mathrm{E}+00$ & $<3.04 \mathrm{E}+01$ & $5.37 \mathrm{E}+03$ & $6.76 \mathrm{E}+03$ & $6.89 \mathrm{E}+03$ \\
\hline $\mathrm{U}(\mathrm{IE})$ & $<2.61 \mathrm{E}+02$ & $<2.61 \mathrm{E}+03$ & $<2.54 \mathrm{E}+02$ & $<2.61 \mathrm{E}+03$ & - \\
\hline $\mathrm{V}(\mathrm{IE})$ & $<3.76 \mathrm{E}+00$ & $<3.76 \mathrm{E}+01$ & $1.11 \mathrm{E}+02$ & $1.03 \mathrm{E}+02$ & - \\
\hline $\mathrm{Zn}(\mathrm{IE})$ & $<7.36 \mathrm{E}+00$ & $<7.36 \mathrm{E}+01$ & $1.74 \mathrm{E}+02$ & $1.96 \mathrm{E}+02$ & $1.61 \mathrm{E}+02$ \\
\hline $\mathrm{Zr}(\mathrm{IE})$ & $<4.40 \mathrm{E}+00$ & - & $6.25 \mathrm{E}+02$ & - & $9.63 \mathrm{E}+02$ \\
\hline $\mathrm{Hg}(\mathrm{CV})$ & $<4.40 \mathrm{E}+01$ & - & $<4.28 \mathrm{E}+01$ & - & - \\
\hline${ }^{232} \mathrm{Th}(\mathrm{IM})$ & $<1.00 \mathrm{E}+00$ & $<1.00 \mathrm{E}+00$ & $1.83 \mathrm{E}+00$ & $2.99 \mathrm{E}+00$ & - \\
\hline${ }^{233} \mathrm{U}(\mathrm{IM})$ & $<1.00 \mathrm{E}+00$ & $<1.00 \mathrm{E}+00$ & $<9.73 \mathrm{E}-01$ & $<1.00 \mathrm{E}+00$ & - \\
\hline${ }^{234} \mathrm{U}(\mathrm{IM})$ & $<1.00 \mathrm{E}+00$ & $<1.00 \mathrm{E}+00$ & $<9.73 \mathrm{E}-01$ & $<1.00 \mathrm{E}+00$ & - \\
\hline${ }^{235} \mathrm{U}(\mathrm{IM})$ & $<1.50 \mathrm{E}+00$ & $<1.00 \mathrm{E}+00$ & $<1.46 \mathrm{E}+00$ & $<1.00 \mathrm{E}+00$ & - \\
\hline${ }^{236} \mathrm{U}(\mathrm{IM})$ & $<1.00 \mathrm{E}+00$ & $<2.00 \mathrm{E}+00$ & $<9.73 \mathrm{E}-01$ & $<2.00 \mathrm{E}+00$ & - \\
\hline${ }^{238} \mathrm{U}(\mathrm{IM})$ & $<1.00 \mathrm{E}+00$ & $1.38 \mathrm{E}+00$ & $6.30 \mathrm{E}+00$ & $7.31 \mathrm{E}+00$ & - \\
\hline $\mathrm{U}-\mathrm{total}(\mathrm{IM})$ & $<1.00 \mathrm{E}+00$ & $1.38 \mathrm{E}+00$ & $7.76 \mathrm{E}+00$ & $7.31 \mathrm{E}+00$ & - \\
\hline${ }^{90} \mathrm{Sr}(\mathrm{BS})$ & $<5.82 \mathrm{E}-03$ & $<5.95 \mathrm{E}-03$ & $<7.52 \mathrm{E}-03$ & $<6.90 \mathrm{E}-03$ & - \\
\hline${ }^{137} \mathrm{Cs}(\mathrm{GS})$ & $<3.20 \mathrm{E}-02$ & $<3.41 \mathrm{E}-02$ & $<3.72 \mathrm{E}-02$ & $<3.99 \mathrm{E}-02$ & - \\
\hline${ }^{238} \mathrm{Pu}(\mathrm{SA})$ & $<9.08 \mathrm{E}-05$ & $<4.42 \mathrm{E}-05$ & $<7.18 \mathrm{E}-05$ & $9.34 \mathrm{E}-05$ & - \\
\hline${ }^{99} \mathrm{Tc}(\mathrm{IM})$ & $<1.00 \mathrm{E}+00$ & $<1.00 \mathrm{E}+00$ & $<9.73 \mathrm{E}-01$ & $<1.00 \mathrm{E}+00$ & - \\
\hline${ }^{237} \mathrm{~Np}(\mathrm{IM})$ & $<1.00 \mathrm{E}+00$ & $<2.50 \mathrm{E}+00$ & $<9.73 \mathrm{E}-01$ & $<2.50 \mathrm{E}+00$ & - \\
\hline${ }^{239} \mathrm{Pu}(\mathrm{IM})$ & $<2.00 \mathrm{E}+00$ & $<1.00 \mathrm{E}+00$ & $<1.95 \mathrm{E}+00$ & $<1.00 \mathrm{E}+00$ & - \\
\hline$\left.{ }^{240} \mathrm{Pu}\right)$ & $<1.00 \mathrm{E}+00$ & $<2.00 \mathrm{E}+00$ & $<9.73 \mathrm{E}-01$ & $<2.00 \mathrm{E}+00$ & - \\
\hline
\end{tabular}

Values in italics were not used in calculating the average. Divide $\mathrm{mg} / \mathrm{kg}$ values by $1 \mathrm{E}+04$ to convert to wt $\%$ basis. 
SRNL-STI-2012-00309

Revision 0

Table A-1. Composition of Blanks and Standards for the Water Leached Solids from Tank 16H Annulus Sample (continued)

\begin{tabular}{|l|c|c|c|c|c||}
\hline Analyte (Method) & $\begin{array}{c}\text { Aqua Regia } \\
\text { Blank } \\
(\mathbf{d p m} / \mathbf{g})\end{array}$ & $\begin{array}{c}\text { Per Fusion } \\
\text { Blank } \\
(\mathbf{d p m} / \mathbf{g})\end{array}$ & $\begin{array}{c}\text { Aqua Regia } \\
\text { Glass Std } \\
\text { (dpm/g) }\end{array}$ & $\begin{array}{c}\text { Per Fusion } \\
\text { Glass Std } \\
\text { (dpm/g) }\end{array}$ & $\begin{array}{c}\text { Glass Std } \\
\text { Composition } \\
\text { (dpm/g) }\end{array}$ \\
\hline${ }^{90} \mathrm{Sr}(\mathrm{BS})$ & $<1.77 \mathrm{E}+06$ & $<1.81 \mathrm{E}+06$ & $<2.29 \mathrm{E}+06$ & $<2.10 \mathrm{E}+06$ & - \\
\hline${ }^{137} \mathrm{Cs}(\mathrm{GS})$ & $<6.16 \mathrm{E}+06$ & $<6.56 \mathrm{E}+06$ & $<7.16 \mathrm{E}+06$ & $<7.68 \mathrm{E}+06$ & - \\
\hline${ }^{238} \mathrm{Pu}(\mathrm{SA})$ & $<3.45 \mathrm{E}+03$ & $<1.68 \mathrm{E}+03$ & $<2.73 \mathrm{E}+03$ & $3.55 \mathrm{E}+03$ & - \\
\hline${ }^{239 / 240} \mathrm{Pu}(\mathrm{SA})$ & $<3.94 \mathrm{E}+03$ & $<2.84 \mathrm{E}+03$ & $<1.18 \mathrm{E}+03$ & $<3.06 \mathrm{E}+03$ & - \\
\hline${ }^{99} \mathrm{Tc}(\mathrm{IM})$ & $<3.77 \mathrm{E}+04$ & $<3.77 \mathrm{E}+04$ & $<3.66 \mathrm{E}+04$ & $<3.77 \mathrm{E}+04$ & - \\
\hline${ }^{232} \mathrm{Th}(\mathrm{IM})$ & $<2.44 \mathrm{E}-01$ & $<2.44 \mathrm{E}-01$ & $4.46 \mathrm{E}-01$ & $7.31 \mathrm{E}-01$ & - \\
\hline${ }^{233} \mathrm{U}(\mathrm{IM})$ & $<2.14 \mathrm{E}+04$ & $<2.14 \mathrm{E}+04$ & $<2.08 \mathrm{E}+04$ & $<2.14 \mathrm{E}+04$ & - \\
\hline${ }^{234} \mathrm{U}(\mathrm{IM})$ & $<1.39 \mathrm{E}+04$ & $<1.39 \mathrm{E}+04$ & $<1.35 \mathrm{E}+04$ & $<1.39 \mathrm{E}+04$ & - \\
\hline${ }^{235} \mathrm{U}(\mathrm{IM})$ & $<7.19 \mathrm{E}+00$ & $<4.80 \mathrm{E}+00$ & $<7.00 \mathrm{E}+00$ & $<4.80 \mathrm{E}+00$ & - \\
\hline${ }^{236} \mathrm{U}(\mathrm{IM})$ & $<1.44 \mathrm{E}+02$ & $<2.87 \mathrm{E}+02$ & $<1.40 \mathrm{E}+02$ & $<2.87 \mathrm{E}+02$ & - \\
\hline${ }^{237} \mathrm{~Np}(\mathrm{IM})$ & $<1.56 \mathrm{E}+03$ & $<3.91 \mathrm{E}+03$ & $<1.52 \mathrm{E}+03$ & $<3.91 \mathrm{E}+03$ & - \\
\hline${ }^{238} \mathrm{U}(\mathrm{IM})$ & $<7.46 \mathrm{E}-01$ & $1.03 \mathrm{E}+00$ & $4.70 \mathrm{E}+00$ & $5.45 \mathrm{E}+00$ & - \\
\hline${ }^{239} \mathrm{Pu}(\mathrm{IM})$ & $<2.72 \mathrm{E}+05$ & $<1.36 \mathrm{E}+05$ & $<2.65 \mathrm{E}+05$ & $<1.36 \mathrm{E}+05$ & - \\
\hline${ }^{240} \mathrm{Pu}(\mathrm{IM})$ & $<5.01 \mathrm{E}+05$ & $<1.00 \mathrm{E}+06$ & $<4.87 \mathrm{E}+05$ & $<1.00 \mathrm{E}+06$ & - \\
\hline
\end{tabular}

Values in italics were not used in calculating the average. Divide $\mathrm{mg} / \mathrm{kg}$ values by $1 \mathrm{E}+04$ to convert to wt $\%$ basis. 
SRNL-STI-2012-00309

Revision 0

Table A-2. Composition of Blanks and Standards for the Oxalic Acid Leached Solids from Tank 16H Annulus Sample

\begin{tabular}{|c|c|c|c|c|c|}
\hline Analyte (Method) & $\begin{array}{c}\text { Aqua Regia } \\
\text { Blank } \\
\text { (mg/kg) }\end{array}$ & $\begin{array}{c}\text { Per Fusion } \\
\text { Blank } \\
(\mathrm{mg} / \mathrm{kg}) \\
\end{array}$ & $\begin{array}{c}\text { Aqua Regia } \\
\text { Glass Std } \\
\text { (mg/kg) }\end{array}$ & $\begin{array}{c}\text { Per Fusion } \\
\text { Glass Std } \\
\text { (mg/kg) }\end{array}$ & $\begin{array}{c}\text { Glass Std } \\
\text { Composition } \\
(\mathrm{mg} / \mathrm{kg})\end{array}$ \\
\hline $\mathrm{Ag}(\mathrm{IE})$ & $<1.15 \mathrm{E}+02$ & $<1.15 \mathrm{E}+03$ & $<1.07 \mathrm{E}+02$ & $<1.05 \mathrm{E}+03$ & - \\
\hline $\mathrm{Al}$ (IE) & $5.42 \mathrm{E}+01$ & $2.31 \mathrm{E}+03$ & $2.40 \mathrm{E}+04$ & $2.46 \mathrm{E}+04$ & $2.50 \mathrm{E}+04$ \\
\hline B (IE) & $<1.62 \mathrm{E}+01$ & $<1.62 \mathrm{E}+02$ & $2.62 \mathrm{E}+04$ & $2.49 \mathrm{E}+04$ & $2.69 \mathrm{E}+04$ \\
\hline $\mathrm{Ba}$ (IE) & $<4.16 \mathrm{E}+00$ & $1.26 \mathrm{E}+02$ & $8.04 \mathrm{E}+02$ & $8.21 \mathrm{E}+02$ & $7.88 \mathrm{E}+02$ \\
\hline Be (IE) & $<4.80 \mathrm{E}-01$ & $<6.40 \mathrm{E}+00$ & $2.13 \mathrm{E}+01$ & $2.69 \mathrm{E}+01$ & - \\
\hline $\mathrm{Ca}$ (IE) & $3.73 \mathrm{E}+01$ & $3.53 \mathrm{E}+03$ & $1.08 \mathrm{E}+04$ & $1.29 \mathrm{E}+04$ & $1.02 \mathrm{E}+04$ \\
\hline $\mathrm{Cd}$ (IE) & $<2.48 \mathrm{E}+00$ & $<2.48 \mathrm{E}+01$ & $3.57 \mathrm{E}+00$ & $<2.25 \mathrm{E}+01$ & - \\
\hline Ce (IE) & $<5.66 \mathrm{E}+01$ & $<5.66 \mathrm{E}+02$ & $<5.26 \mathrm{E}+01$ & $<5.14 \mathrm{E}+02$ & - \\
\hline Co (IE) & $<7.76 \mathrm{E}+00$ & $<6.80 \mathrm{E}+01$ & $6.22 \mathrm{E}+01$ & $8.29 \mathrm{E}+01$ & - \\
\hline $\mathrm{Cr}$ (IE) & $<6.56 \mathrm{E}+00$ & $<6.56 \mathrm{E}+01$ & $7.08 \mathrm{E}+02$ & $6.73 \mathrm{E}+02$ & $6.36 \mathrm{E}+02$ \\
\hline $\mathrm{Cu}$ (IE) & $<5.04 \mathrm{E}+00$ & $<5.04 \mathrm{E}+01$ & $1.53 \mathrm{E}+01$ & $<4.58 \mathrm{E}+01$ & $3.20 \mathrm{E}+01$ \\
\hline $\mathrm{Fe}$ (IE) & $2.48 \mathrm{E}+01$ & $2.54 \mathrm{E}+02$ & $9.78 \mathrm{E}+04$ & $9.60 \mathrm{E}+04$ & $9.79 \mathrm{E}+04$ \\
\hline Gd (IE) & $<1.72 \mathrm{E}+01$ & $<1.72 \mathrm{E}+02$ & $<1.60 \mathrm{E}+01$ & $<1.56 \mathrm{E}+02$ & - \\
\hline K (IE) & $<2.05 \mathrm{E}+02$ & $<2.05 \mathrm{E}+03$ & $2.25 \mathrm{E}+04$ & $2.42 \mathrm{E}+04$ & $2.25 \mathrm{E}+04$ \\
\hline $\mathrm{La}$ (IE) & $<5.36 \mathrm{E}+00$ & $<5.36 \mathrm{E}+01$ & $<4.98 \mathrm{E}+00$ & $<4.87 \mathrm{E}+01$ & - \\
\hline $\mathrm{Li}$ (IE) & $<1.17 \mathrm{E}+01$ & $<1.17 \mathrm{E}+02$ & $1.46 \mathrm{E}+04$ & $1.40 \mathrm{E}+04$ & $1.49 \mathrm{E}+04$ \\
\hline $\mathrm{Mg}$ (IE) & $4.84 \mathrm{E}+00$ & $1.28 \mathrm{E}+01$ & $5.24 \mathrm{E}+03$ & $5.12 \mathrm{E}+03$ & $5.19 \mathrm{E}+03$ \\
\hline Mn (IE) & $<4.24 \mathrm{E}+00$ & $<1.68 \mathrm{E}+01$ & $1.44 \mathrm{E}+04$ & $1.41 \mathrm{E}+04$ & $1.46 \mathrm{E}+04$ \\
\hline Mo (IE) & $<4.94 \mathrm{E}+01$ & $<2.14 \mathrm{E}+02$ & $<4.59 \mathrm{E}+01$ & $<1.95 \mathrm{E}+02$ & - \\
\hline $\mathrm{Na}$ (IE) & $8.77 \mathrm{E}+02$ & - & $8.52 \mathrm{E}+04$ & - & $8.53 \mathrm{E}+04$ \\
\hline Ni (IE) & $<3.64 \mathrm{E}+01$ & $<3.64 \mathrm{E}+02$ & $8.06 \mathrm{E}+03$ & $7.82 \mathrm{E}+03$ & $8.25 \mathrm{E}+03$ \\
\hline $\mathrm{P}$ (IE) & $<6.93 \mathrm{E}+01$ & $<1.31 \mathrm{E}+03$ & $1.11 \mathrm{E}+03$ & $1.27 \mathrm{E}+03$ & $9.60 \mathrm{E}+02$ \\
\hline $\mathrm{Pb}$ (IE) & $<5.73 \mathrm{E}+01$ & $<5.73 \mathrm{E}+02$ & $<5.32 \mathrm{E}+01$ & $<5.21 \mathrm{E}+02$ & - \\
\hline $\mathrm{S}$ (IE) & $<6.00 \mathrm{E}+02$ & $<6.00 \mathrm{E}+03$ & $<5.58 \mathrm{E}+02$ & $<5.45 \mathrm{E}+03$ & - \\
\hline $\mathrm{Sb}$ (IE) & $<1.02 \mathrm{E}+02$ & $<8.53 \mathrm{E}+02$ & $1.63 \mathrm{E}+02$ & $<7.75 \mathrm{E}+02$ & - \\
\hline Si (IE) & - & $<3.51 \mathrm{E}+02$ & - & $1.57 \mathrm{E}+05$ & $2.24 \mathrm{E}+05$ \\
\hline Sn (IE) & $<4.49 \mathrm{E}+01$ & $<4.49 \mathrm{E}+02$ & $6.30 \mathrm{E}+01$ & $<4.08 \mathrm{E}+02$ & - \\
\hline
\end{tabular}

Values in italics were not used in calculating the average. Divide $\mathrm{mg} / \mathrm{kg}$ values by $1 \mathrm{E}+04$ to convert to wt $\%$ basis. 
Table A-2. Composition of Blanks and Standards for the Oxalic Acid Leached Solids from Tank 16H Annulus Sample (continued)

\begin{tabular}{|c|c|c|c|c|c|}
\hline Analyte (Method) & $\begin{array}{c}\text { Aqua Regia } \\
\text { Blank } \\
\text { (mg/kg) }\end{array}$ & $\begin{array}{c}\text { Per Fusion } \\
\text { Blank } \\
\text { (mg/kg) }\end{array}$ & $\begin{array}{c}\text { Aqua Regia } \\
\text { Glass Std } \\
\text { (mg/kg) }\end{array}$ & $\begin{array}{c}\text { Per Fusion } \\
\text { Glass Std } \\
\text { (mg/kg) }\end{array}$ & $\begin{array}{c}\text { Glass Std } \\
\text { Composition } \\
\text { (mg/kg) }\end{array}$ \\
\hline $\operatorname{Sr}(\mathrm{IE})$ & $<4.00 \mathrm{E}-01$ & $3.60 \mathrm{E}+01$ & $3.25 \mathrm{E}+01$ & $5.89 \mathrm{E}+01$ & $3.13 \mathrm{E}+01$ \\
\hline Th (IE) & $<4.28 \mathrm{E}+01$ & $<2.14 \mathrm{E}+02$ & $<3.98 \mathrm{E}+01$ & $<1.95 \mathrm{E}+02$ & - \\
\hline Ti (IE) & $<1.04 \mathrm{E}+00$ & $<1.04 \mathrm{E}+01$ & $4.50 \mathrm{E}+03$ & $6.76 \mathrm{E}+03$ & $6.89 \mathrm{E}+03$ \\
\hline U (IE) & $<2.61 \mathrm{E}+02$ & $<2.61 \mathrm{E}+03$ & $<2.43 \mathrm{E}+03$ & $<2.37 \mathrm{E}+03$ & - \\
\hline V (IE) & $<3.76 \mathrm{E}+00$ & $<3.76 \mathrm{E}+01$ & $1.09 \mathrm{E}+02$ & $1.03 \mathrm{E}+02$ & - \\
\hline Zn (IE) & $1.31 \mathrm{E}+01$ & $<5.28 \mathrm{E}+01$ & $1.67 \mathrm{E}+02$ & $2.29 \mathrm{E}+02$ & $1.61 \mathrm{E}+02$ \\
\hline $\mathrm{Zr}$ (IE) & $6.56 \mathrm{E}+00$ & - & $4.09 \mathrm{E}+02$ & - & $9.63 \mathrm{E}+02$ \\
\hline $\mathrm{Hg}(\mathrm{CV})$ & $<4.40 \mathrm{E}+01$ & - & $<4.01 \mathrm{E}+01$ & - & - \\
\hline${ }^{232} \mathrm{Th}$ (IM) & $<2.00 \mathrm{E}-01$ & $<1.20 \mathrm{E}+00$ & $1.48 \mathrm{E}+00$ & $2.09 \mathrm{E}+00$ & - \\
\hline${ }^{233} \mathrm{U}(\mathrm{IM})$ & $<2.00 \mathrm{E}-01$ & $<8.00 \mathrm{E}-01$ & $<1.86 \mathrm{E}-01$ & $<7.27 \mathrm{E}-01$ & - \\
\hline${ }^{234} \mathrm{U}$ (IM) & $<2.00 \mathrm{E}-01$ & $<8.00 \mathrm{E}-01$ & $<1.86 \mathrm{E}-01$ & $<7.27 \mathrm{E}-01$ & - \\
\hline${ }^{235} \mathrm{U}$ (IM) & $<2.00 \mathrm{E}-01$ & $<8.00 \mathrm{E}-01$ & $<1.86 \mathrm{E}-01$ & $<7.27 \mathrm{E}-01$ & - \\
\hline${ }^{236} \mathrm{U}$ (IM) & $<2.00 \mathrm{E}-01$ & $<8.00 \mathrm{E}-01$ & $<1.86 \mathrm{E}-01$ & $<7.27 \mathrm{E}-01$ & - \\
\hline${ }^{238} \mathrm{U}$ (IM) & $<5.00 \mathrm{E}-01$ & $<1.20 \mathrm{E}+00$ & $4.84 \mathrm{E}+00$ & $5.49 \mathrm{E}+00$ & - \\
\hline U-total (IM) & $<1.30 \mathrm{E}+00$ & $<4.40 \mathrm{E}+00$ & $4.84 \mathrm{E}+00$ & $5.49 \mathrm{E}+00$ & - \\
\hline${ }^{90} \mathrm{Sr}$ (BS) & $<7.03 \mathrm{E}-03$ & $<6.08 \mathrm{E}-03$ & $<5.42 \mathrm{E}-03$ & $<6.47 \mathrm{E}-03$ & - \\
\hline${ }^{137} \mathrm{Cs}$ (GS) & $<3.74 \mathrm{E}-02$ & $<2.53 \mathrm{E}-02$ & $<3.79 \mathrm{E}-02$ & $<3.00 \mathrm{E}-02$ & - \\
\hline${ }^{238} \mathrm{Pu}$ (SA) & $<2.18 \mathrm{E}-04$ & $<8.95 \mathrm{E}-05$ & $<7.18 \mathrm{E}-05$ & 7.47E-05 & - \\
\hline${ }^{99} \mathrm{Tc}$ (IM) & $<3.00 \mathrm{E}-01$ & $<8.00 \mathrm{E}-01$ & $<2.79 \mathrm{E}-01$ & 7.27E-01 & - \\
\hline${ }^{237} \mathrm{~Np}$ (IM) & $<2.00 \mathrm{E}-01$ & $<8.00 \mathrm{E}-01$ & $<1.86 \mathrm{E}-01$ & $<7.27 \mathrm{E}-01$ & - \\
\hline${ }^{239} \mathrm{Pu}$ (IM) & $<2.00 \mathrm{E}-01$ & $<2.00 \mathrm{E}+00$ & $<1.86 \mathrm{E}-01$ & $<1.82 \mathrm{E}+00$ & - \\
\hline${ }^{240} \mathrm{Pu}$ (IM) & $<2.00 \mathrm{E}-01$ & $<8.00 \mathrm{E}-01$ & $<1.86 \mathrm{E}-01$ & $<7.27 \mathrm{E}-01$ & - \\
\hline
\end{tabular}

Values in italics were not used in calculating the average. Divide $\mathrm{mg} / \mathrm{kg}$ values by $1 \mathrm{E}+04$ to convert to wt $\%$ basis. 
Table A-2. Composition of Blanks and Standards for the Oxalic Acid Leached Solids from Tank 16H Annulus Sample (continued)

\begin{tabular}{||l|c|c|c|c|c||}
\hline Analyte (Method) & $\begin{array}{c}\text { Aqua Regia } \\
\text { Blank } \\
\text { (dpm/g) }\end{array}$ & $\begin{array}{c}\text { Per Fusion } \\
\text { Blank } \\
\text { (dpm/g) }\end{array}$ & $\begin{array}{c}\text { Aqua Regia } \\
\text { Glass Std } \\
\text { (dpm/g) }\end{array}$ & $\begin{array}{c}\text { Per Fusion } \\
\text { Glass Std } \\
\text { (dpm/g) }\end{array}$ & $\begin{array}{c}\text { Glass Std } \\
\text { Composition } \\
\text { (dpm/g) }\end{array}$ \\
\hline${ }^{90} \mathrm{Sr}(\mathrm{BS})$ & $<2.14 \mathrm{E}+06$ & $<1.85 \mathrm{E}+06$ & $<1.65 \mathrm{E}+06$ & $<1.97 \mathrm{E}+06$ & - \\
\hline${ }^{137} \mathrm{Cs}(\mathrm{GS})$ & $<7.20 \mathrm{E}+06$ & $<4.88 \mathrm{E}+06$ & $<7.29 \mathrm{E}+06$ & $<5.78 \mathrm{E}+06$ & - \\
\hline${ }^{238} \mathrm{Pu}(\mathrm{SA})$ & $<8.29 \mathrm{E}+03$ & $<3.40 \mathrm{E}+03$ & $<2.73 \mathrm{E}+03$ & $<2.84 \mathrm{E}+03$ & - \\
\hline${ }^{239 / 240} \mathrm{Pu}(\mathrm{SA})$ & $2.89 \mathrm{E}+03$ & $<3.85 \mathrm{E}+03$ & $<2.63 \mathrm{E}+03$ & $<2.49 \mathrm{E}+03$ & - \\
\hline${ }^{239} \mathrm{Tc}(\mathrm{IM})$ & $<1.13 \mathrm{E}+04$ & $<3.01 \mathrm{E}+04$ & $<1.05 \mathrm{E}+04$ & $2.74 \mathrm{E}+04$ & - \\
\hline${ }^{233} \mathrm{U}(\mathrm{IM})$ & $<4.88 \mathrm{E}-02$ & $<2.93 \mathrm{E}-01$ & $3.62 \mathrm{E}-01$ & $5.11 \mathrm{E}-01$ & - \\
\hline${ }^{234} \mathrm{U}(\mathrm{IM})$ & $<4.28 \mathrm{E}+03$ & $<1.71 \mathrm{E}+04$ & $<3.98 \mathrm{E}+03$ & $<1.56 \mathrm{E}+04$ & - \\
\hline${ }^{235} \mathrm{U}(\mathrm{IM})$ & $<2.78 \mathrm{E}+03$ & $<1.11 \mathrm{E}+04$ & $<2.58 \mathrm{E}+03$ & $<1.01 \mathrm{E}+04$ & - \\
\hline${ }^{236} \mathrm{U}(\mathrm{IM})$ & $<9.59 \mathrm{E}-01$ & $<3.84 \mathrm{E}+00$ & $<8.91 \mathrm{E}-01$ & $<3.49 \mathrm{E}+00$ & - \\
\hline${ }^{237} \mathrm{~Np}(\mathrm{IM})$ & $<2.87 \mathrm{E}+01$ & $<1.15 \mathrm{E}+02$ & $<2.67 \mathrm{E}+01$ & $<1.04 \mathrm{E}+02$ & - \\
\hline${ }^{238} \mathrm{U}(\mathrm{IM})$ & $<3.13 \mathrm{E}+02$ & $<1.25 \mathrm{E}+03$ & $<2.91 \mathrm{E}+02$ & $<1.14 \mathrm{E}+03$ & - \\
\hline${ }^{239} \mathrm{Pu}(\mathrm{IM})$ & $<3.73 \mathrm{E}-01$ & $<8.95 \mathrm{E}-01$ & $3.61 \mathrm{E}+00$ & $4.09 \mathrm{E}+00$ & - \\
\hline $240 \mathrm{IM})$ & $<2.72 \mathrm{E}+04$ & $<2.72 \mathrm{E}+05$ & $<2.53 \mathrm{E}+04$ & $<2.48 \mathrm{E}+05$ & - \\
\hline
\end{tabular}

Values in italics were not used in calculating the average. Divide $\mathrm{mg} / \mathrm{kg}$ values by $1 \mathrm{E}+04$ to convert to wt $\%$ basis. 